\title{
An Efficient and Stable Hydrophobic Molecular Cobalt Catalyst for Water Electrooxidation at Neutral pH
}

\author{
Ba-Tian Chen, ${ }^{\dagger \neq}$ Natalia Morlanés, ${ }^{+\ddagger}$ Enoch Adogla, ${ }^{\star}$ Kazuhiro Takanabe, ${ }^{\dagger}$ Valentin O. Rodionov ${ }^{+*}$ \\ + KAUST Catalysis Center and Division of Physical Sciences and Engineering, King Abdullah University of Science and Technology \\ (KAUST), Thuwal, 23955-6900, Kingdom of Saudi Arabia. \\ \# Department of Chemistry and Biochemistry, University of South Carolina, 631 Sumter Street, Columbia, SC 29208, USA. \\ *E-mail: valentin.rodionov@kaust.edu.sa \\ Tel: +966 (12) 8084592
}

₹ B-TC and NM contributed equally. 


\section{Contents}

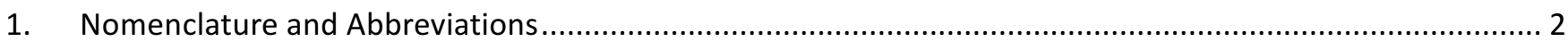

2. Reported Cobalt-Based Molecular Catalysts for Water Oxidation ............................................................ 3

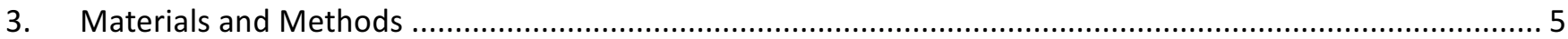

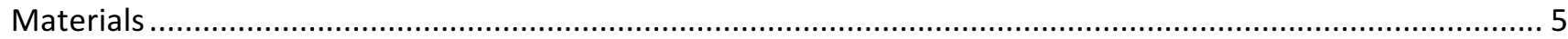

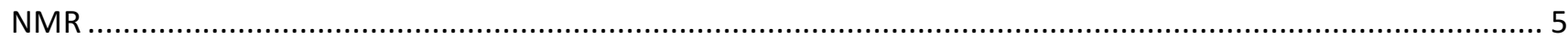

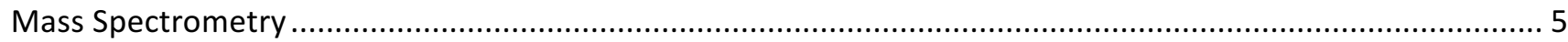

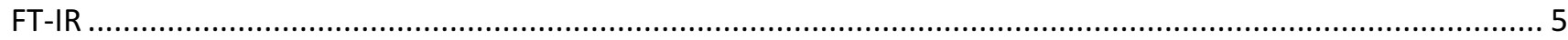

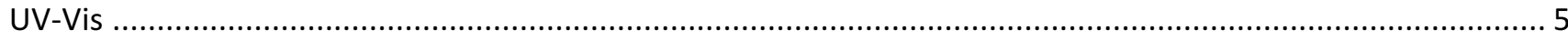

ICP-OES …

Contact Angle

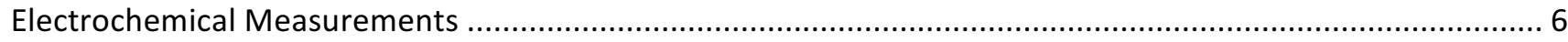

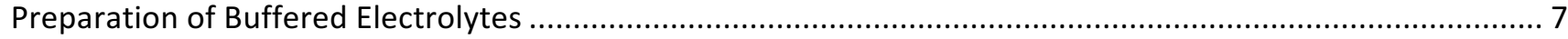

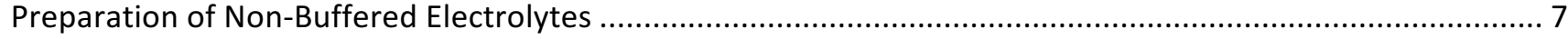

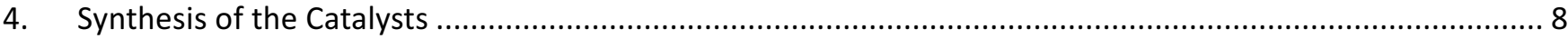

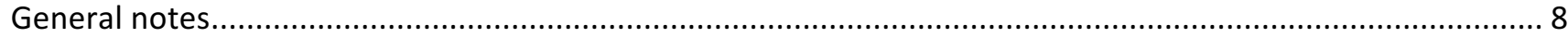

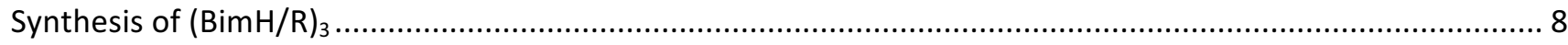

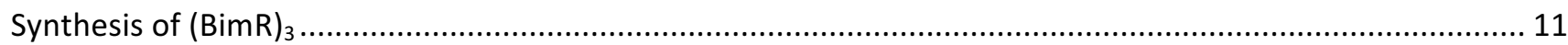

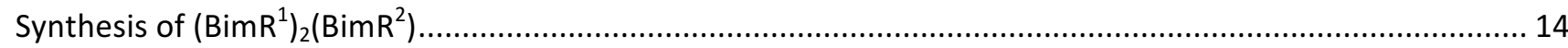

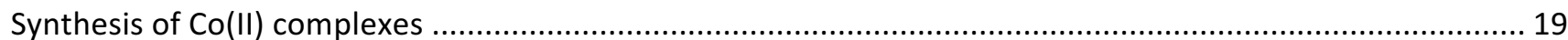

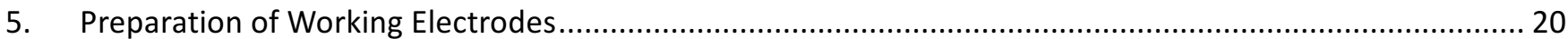

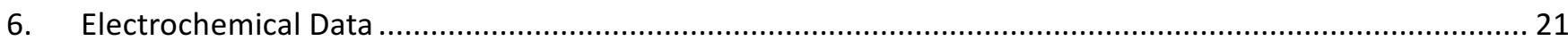

7. Spectroscopic Characterization of the Co-complexes after electrochemistry ........................................... 30

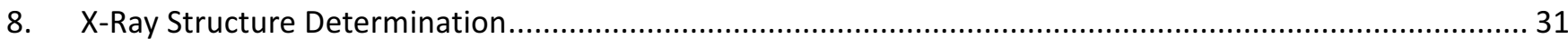

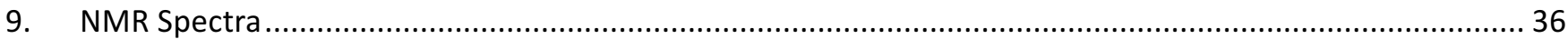

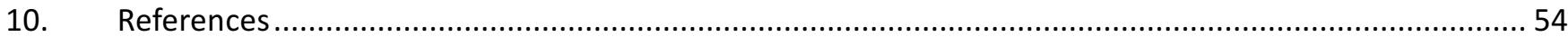




\section{Nomenclature and Abbreviations}

Table S1. Nomenclature and abbreviations

\begin{tabular}{|c|c|}
\hline Abbreviation & Name/description \\
\hline ATR-FTIR & attenuated total reflectance Fourier transform infrared spectroscopy \\
\hline $\mathrm{CPE}$ & controlled potential electrolysis \\
\hline $\mathrm{CV}$ & cyclic voltammogram \\
\hline ESI & electrospray ionization \\
\hline FE & Faradaic efficiency \\
\hline FTO & fluorine-doped tin oxide \\
\hline HRMS & high resolution mass spectrometry \\
\hline ICP-OES & inductively coupled plasma optical emission spectrometry catalytic \\
\hline $\mathrm{i}_{\text {cat }}$ & current density \\
\hline $\mathrm{i}_{\text {diff }}$ & diffusional current density \\
\hline iR & internal resistance \\
\hline LC-MS & liquid chromatography - mass spectrometry \\
\hline NMR & nuclear-magnetic resonance \\
\hline NHE & normal hydrogen electrode \\
\hline RHE & reversible hydrogen electrode \\
\hline TCD & thermal conductivity detector \\
\hline THF & tetrahydrofuran \\
\hline TON & turnover number \\
\hline TOF & turnover frequency \\
\hline UV-Vis & ultraviolet- visible absorption spectroscopy \\
\hline WO & water oxidation \\
\hline WOC & water-oxidation catalyst \\
\hline$\Gamma$ & surface loading, $\mathrm{mol} / \mathrm{cm}^{2}$ \\
\hline
\end{tabular}




\section{Reported Cobalt-Based Molecular Catalysts for Water Oxidation}

Table S2. Summary of previously reported cobalt-based molecular catalysts for water-oxidation reactions ${ }^{[a]}$

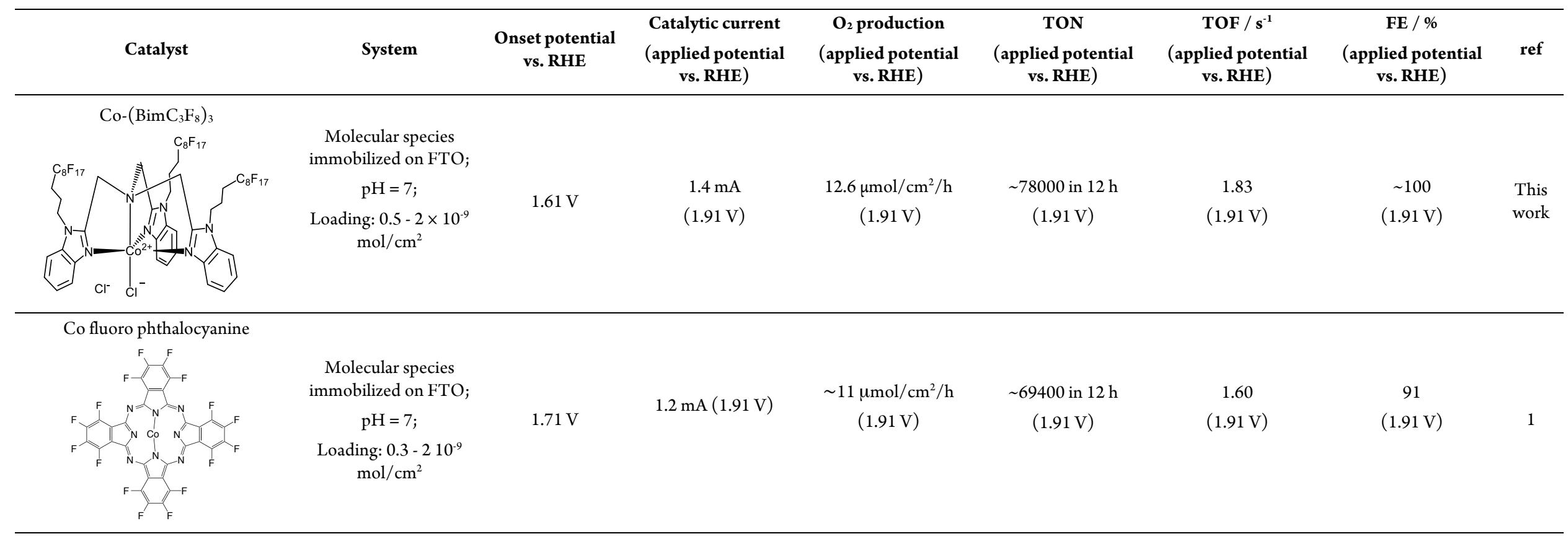

Co-cationic porphyrin

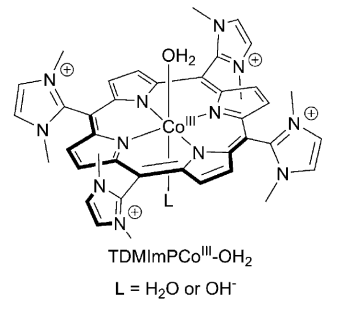

Homogeneous aqueous solution; $\mathrm{pH}=7$; $1.81 \mathrm{~V}$

$0.7 \mathrm{~mA}$

$(2.21 \mathrm{~V})$
$10.2 \mu \mathrm{mol} / \mathrm{cm}^{2} / \mathrm{h}$
(1.91 V)

$85-90$

Loading: $1 \mathrm{mM}$

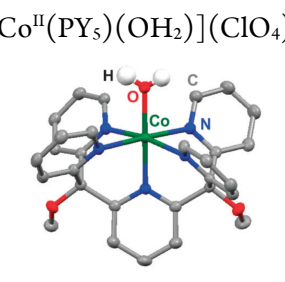

\section{Homogeneous}

aqueous solution;

$\mathrm{pH}=9.2 ;$

$1.94 \mathrm{~V}$

$(2.14 \mathrm{~V})$

NR 


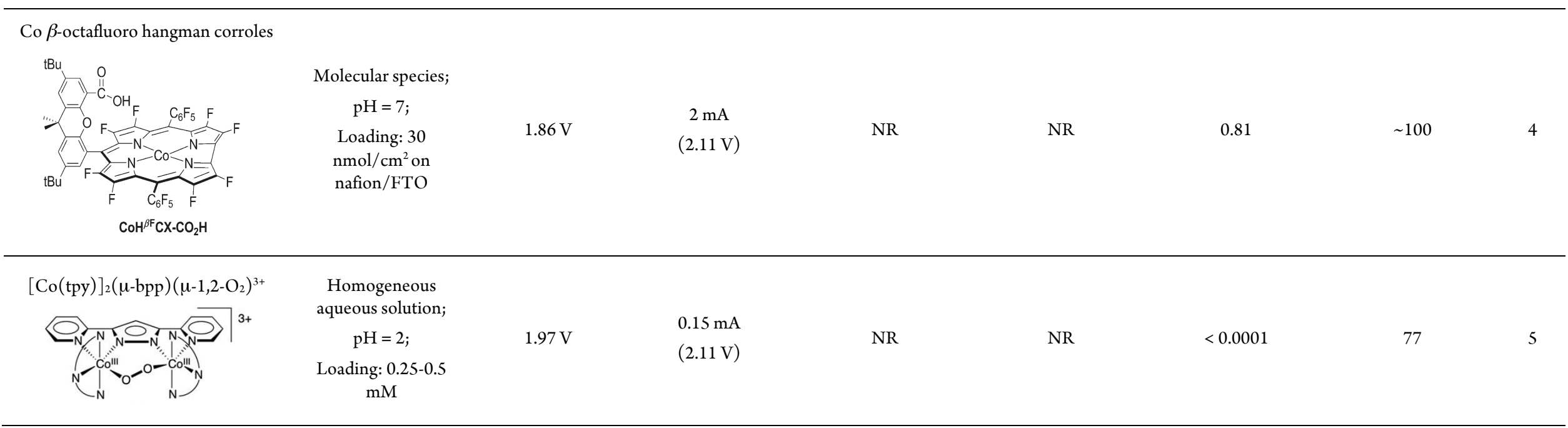

${ }^{[a]}$ All reported potentials were converted to values vs. $\mathrm{RHE}$ for easy comparison using the following formulae: $\mathrm{E}(\mathrm{NHE})=\mathrm{E}(\mathrm{Ag} / \mathrm{AgCl})+0.197 \mathrm{~V} ; \mathrm{E}(\mathrm{RHE})=\mathrm{E}(\mathrm{NHE})+0.0591 \times \mathrm{pH} .{ }^{[\mathrm{b}]} \mathrm{Not}$ reported. 


\section{Materials and Methods}

\section{Materials}

All reagents and solvents were provided by commercial suppliers (Sigma-Aldrich, Fisher Scientific, Alfa Aesar and VWR) and used without further purification unless otherwise noted.

\section{NMR}

${ }^{1} \mathrm{H},{ }^{13} \mathrm{C}$, and ${ }^{19} \mathrm{~F}$ spectra were obtained at $298 \mathrm{~K}$ using Bruker AVQ $700 \mathrm{MHz}, 600 \mathrm{MHz}, 500 \mathrm{MHz}$ or $400 \mathrm{MHz}$ instruments. Chemical shifts are reported as $\delta(\mathrm{ppm})$ values, using either TMS or residual solvent peaks as an internal standard and coupling constants $(J)$ as $\mathrm{Hz}$ values.

\section{Mass Spectrometry}

Mass spectra are acquired using electrospray ionization with an Agilent 6130 Quadrupole LC-MS instrument. HRMS analyses were performed in the Analytical Core Lab, 4700 King Abdullah University of Science and Technology (KAUST), Thuwal, 23955-6900, Saudi Arabia.

\section{$F T-I R$}

A Thermo Scientific Nicolet 6700 instrument equipped with nitrogen purge and an ATR accessory was used to collect the data. The instrument was calibrated before sampling against a newly cleaned (acetone) and dried crystal surface. Thirty-two scans from 4000 to $550 \mathrm{~cm}^{-1}$ were recorded for each sample.

\section{UV-Vis}

UV-Vis spectra were recorded using a JASCO spectrophotometer at room temperature. The wavelength was recorded from 800-200 $\mathrm{nm}$ at a scan speed of $400 \mathrm{~nm} / \mathrm{min}$ and a data interval of $1 \mathrm{~nm}$.

\section{ICP-OES}

Cobalt content in the electrodes was measured using inductively coupled plasma optical emission spectrometry. Cobalt standards (from $1000 \mu \mathrm{g} \mathrm{mL}^{-1}$ Specpure AAS standard solution) were prepared for the calibration of the instrument, and linear calibration curves were obtained in the range of 1-50 Co ppb. Quantitative removal of Co from the FTO electrodes was achieved using $0.7 \mathrm{~mL}$ aqua regia $\left(\mathrm{HCl}: \mathrm{HNO}_{3}=3: 1\right)$ and sonication. Samples were then diluted to $7 \mathrm{~mL}$ of deionized water. Multiple trials were averaged to obtain accurate results. 


\section{Contact Angle}

Contact angles were measured using a contact angle goniometer (KRUSS EasyDrop Standard) at ambient pressure and temperature; static angle measurements were made from $1 \mu \mathrm{l}$ drops of deionized water on the surface of silicon wafer slides with deposited Cotrisbenzimidazole complexes.

\section{Electrochemical Measurements}

Electrochemical measurements were performed on a BioLogic Science Instrument potentiostat electrochemical workstation in a glass cell equipped with an FTO working electrode (effective area $1 \mathrm{~cm}^{2}$ ), a spiral Pt counter electrode and an $\mathrm{Ag} / \mathrm{AgCl}$ (in saturated $\mathrm{KCl}$ electrolyte) reference electrode. Cyclic voltammetry $(\mathrm{CV})$ and controlled potential bulk electrolysis $(\mathrm{CPE})$ were performed in $\mathrm{pH}-$ buffered aqueous solutions at room temperature. The water used in all experiments was distilled and deionized by a Milli- $Q$ system from Millipore; $\mathrm{pH}$ was measured using a pH electrode from Fisher Scientific. In the Main Text, CPE results are shown without iR compensation. The CV and CPE results reported in the Supporting Information when indicated are shown with potentials corrected after iR compensation determined by impedance spectroscopy.

In our experiments we have been operating with just $0.5-2$ nmoles of surface-deposited Co complex placed in $\sim 10 \mathrm{~mL}$ of electrolyte in a sealed cell. Thus, the highest bulk solution concentration of Co ions possible is just 50-200 nM, if all the Co-(Bim) ${ }_{3}$ complex is instantly decomposed and converted into a soluble Co compound. One of the important reasons we chose to operate with low loadings of catalysts is to avoid the formation of $\mathrm{CoO}_{\mathrm{x}}$ films that may be catalytically active. A $50-200 \mathrm{nM}$ concentration of Co is usually not nearly sufficient for making enough $\mathrm{CoO}_{\mathrm{x}}$ that will be visible catalytically (see Figure $1 \mathrm{~B}$ of Groves' work ${ }^{2}$ for an example). Typical protocols for deposition of catalytic particulate films call for Co concentrations of at least 2-3 orders of magnitude higher. These same protocols usually call for film deposition over a period of several hours. In contrast, our Co-(Bim) $)_{3} @$ FTO electrodes are immediately electrocatalytically active after drop-casting the complex solutions.

We would also like to bring the reader's attention to the quintessential work ${ }^{6}$ addressing the problem of a mis-identified particulate Cobased WOR catalyst. Figure 2 in this excellent paper ${ }^{6}$ clearly illustrates how the formation of $\mathrm{CoO}_{x}$ can be observed with fairly straightforward electrochemical experiments. Of course, the concentration of leached Co in Finke experiments $(\sim 60 \mu \mathrm{M})$ is three orders of magnitude higher than what is conceivably possible in our case.

Oxygen evolution was measured by GC analysis (TCD detector). The turnover number (TON) and frequency (TOF) were calculated from the amount of oxygen detected and the amount of cobalt loading, yielding a value for the amount of oxygen formed per cobalt and per second.

$\mathrm{TON}=\operatorname{mol~} \mathrm{O}_{2}($ after 12 hour $) / \mathrm{mol} \mathrm{Co}$

$\mathrm{TOF}=\mathrm{mol} \mathrm{O}_{2} / \mathrm{s} / \mathrm{mol} \mathrm{Co}$

Faradaic efficiency was calculated with respect to the theoretical amount of oxygen produced. The amount of oxygen produced was in turn calculated using the steady-state current obtained from controlled potential electrolysis, in which the current is given in electrons passed per second. Assuming water oxidation is a four-electron process, this value is divided by four to give the amount of oxygen formed per second.

Calculation of the theoretical amount of oxygen: 
$\mathrm{mol} \mathrm{O}_{2} / \mathrm{s}=\frac{\mathrm{I}(\mathrm{A})}{F * 4}$

$\mathrm{F}=$ Faradaic constant $=9.64853399 \cdot 10^{4} \mathrm{~A} \cdot \mathrm{s} / \mathrm{mol}$

Faradaic efficiency $=$ measured amount of oxygen $\cdot 100 /$ theoretical amount of oxygen .

\section{Preparation of Buffered Electrolytes}

1. Potassium phosphate $0.1 \mathrm{M}$ buffered electrolyte at different $\mathrm{pH}$ :

In the $\mathrm{pH}$ range between $4-8$, a mixture of potassium phosphate monobasic and potassium phosphate dibasic in the adequate ratios was dissolved in milli-Qwater.

Below $\mathrm{pH}$ 4: $0.1 \mathrm{M}$ solution of monobasic potassium phosphate, $\mathrm{pH}$ was adjusted with $0.1 \mathrm{M}$ phosphoric acid solution ( $\mathrm{pH}=1$ ).

Above $\mathrm{pH}$ 8: $0.1 \mathrm{M}$ solution of dibasic potassium phosphate, $\mathrm{pH}$ was adjusted with $0.1 \mathrm{M}$ potassium hydroxide $(\mathrm{pH}=13.5)$.

2. Potassium phosphate dibasic at different concentrations in the range 0.01-1 $\mathrm{M}$ were prepared from $1 \mathrm{M}$ potassium phosphate dibasic solution. $(\mathrm{pKa}=7.21 \mathrm{pH}=7.8)$

3. Potassium bicarbonate at different concentrations in the range 0.01-1 $\mathrm{M}$ were prepared from $1 \mathrm{M}$ potassium bicarbonate solution. $(\mathrm{pKa}=6.36, \mathrm{pH}=8.9)$

4. Potassium acetate at different concentrations in the range $0.01-1 \mathrm{M}$ were prepared from $1 \mathrm{M}$ potassium acetate solution. $(\mathrm{pKa}=$ 4.74, $\mathrm{pH}=7.7)$

5. Potassium phosphate monobasic at different concentrations in the range 0.01-1 $\mathrm{M}$ were prepared from $1 \mathrm{M}$ potassium phosphate monobasic solution. $(\mathrm{pKa}=2.21, \mathrm{pH}=4.3)$

\section{Preparation of Non-Buffered Electrolytes}

Potassium nitrate at different concentrations in the range 0.01-1 $\mathrm{M}$ were prepared from $1 \mathrm{M}$ potassium nitrate solution.

Potassium perchlorate $0.1 \mathrm{M}$ non-buffered electrolytes:

Below $\mathrm{pH} 7$, $\mathrm{pH}$ was adjusted with $0.1 \mathrm{M}$ perchloric acid solution. $(\mathrm{pH}=0.52)$

Above $\mathrm{pH} 7, \mathrm{pH}$ was adjusted with $0.1 \mathrm{M}$ potassium hydroxide. $(\mathrm{pH}=13.5)$

Potassium sulfate $0.1 \mathrm{M}$ non-buffered electrolyte:

Below $\mathrm{pH} 7$, $\mathrm{pH}$ was adjusted with $0.1 \mathrm{M}$ sulfuric acid solution. $(\mathrm{pH}=1)$

Above $\mathrm{pH} 7, \mathrm{pH}$ was adjusted with $0.1 \mathrm{M}$ potassium hydroxide. $(\mathrm{pH}=13.5)$ 


\section{Synthesis of the Catalysts}

\section{General notes}

Flash chromatography was performed using Merck Kieselgel 60 (230-400 mesh) silica. Reactions requiring anhydrous conditions were performed under argon using standard Schlenk line techniques. Prior to use, THF was purified by refluxing over sodium in the presence of benzophenone until a persistent blue color appeared and then the solvent was collected via distillation. $\left(\mathbf{B i m} \mathbf{C}_{4} \mathbf{C O} \mathbf{O}_{2} \mathbf{K}\right)_{3}$ is commercially available and was purchased from Sigma-Aldrich.

\section{Synthesis of $(\mathrm{BimH} / \mathrm{R})_{3}$}

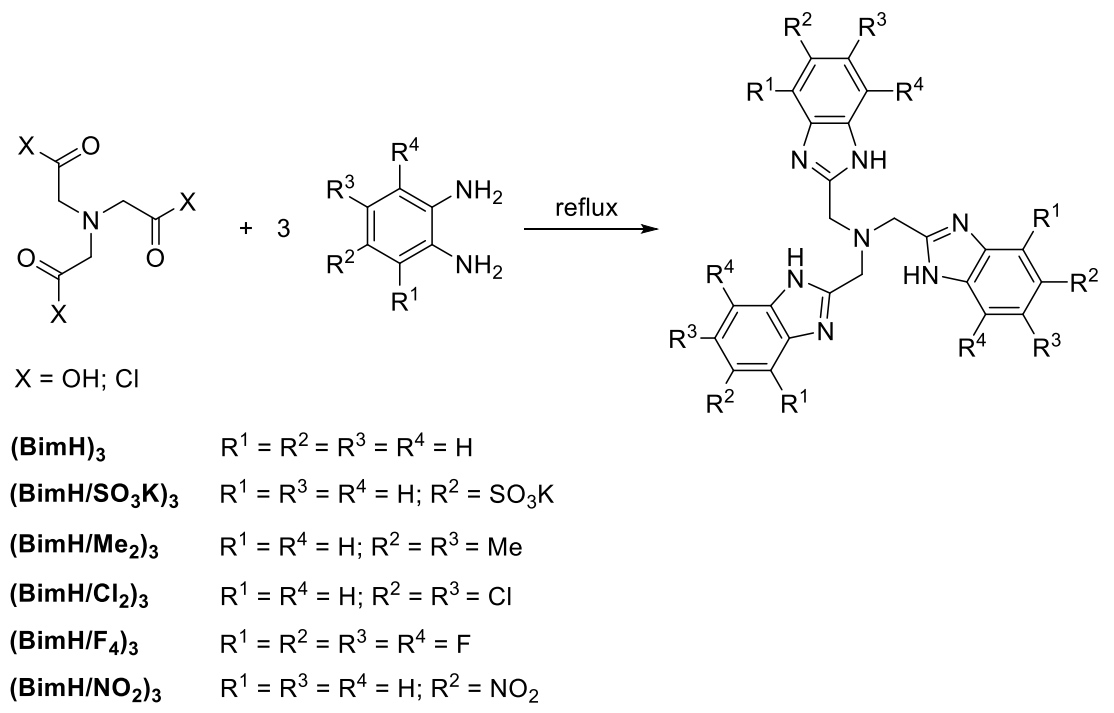

Scheme S1. Synthesis of $(\mathrm{BimH} / \mathbf{R})_{3}$.

$\operatorname{Tris}\left(\left(1 \mathrm{H}-\mathrm{benzo}[\mathrm{d}]\right.\right.$ imidazol-2-yl)methyl)amine, $(\mathrm{BimH})_{3}$

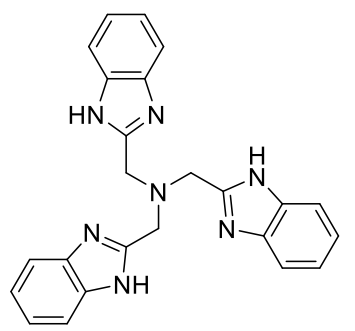

Prepared following a previously reported procedure. ${ }^{7}$

White solid. ${ }^{1} \mathrm{H}$ NMR $\left(700 \mathrm{MHz}, \mathrm{DMSO}-d_{6}\right) \delta 12.44(\mathrm{~s}, 3 \mathrm{H}), 7.63-7.50(\mathrm{~m}, 6 \mathrm{H}), 7.21-7.13(\mathrm{~m}, 6 \mathrm{H}), 4.14(\mathrm{~s}, 6 \mathrm{H}) .{ }^{13} \mathrm{C} \mathrm{NMR}(176$ MHz, DMSO-d $\left.d_{6}\right) \delta 152.14,143.19,134.17,121.97,121.14,118.54,111.24,51.43 . \mathrm{MS}(\mathrm{ESI}) \mathrm{m} / z:[\mathrm{M}+\mathrm{H}]^{+}: 408.2$. 


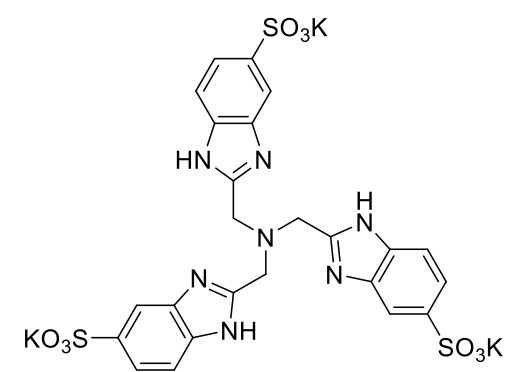

Prepared following a previously reported procedure. ${ }^{8}$

${ }^{1} \mathrm{H}$ NMR $\left(700 \mathrm{MHz}, \mathrm{D}_{2} \mathrm{O}\right) \delta 7.88(\mathrm{br}, 3 \mathrm{H}), 7.48(\mathrm{br}, 3 \mathrm{H}), 7.24(\mathrm{br}, 3 \mathrm{H}), 3.95(\mathrm{br}, 6 \mathrm{H}) .{ }^{13} \mathrm{C} \mathrm{NMR}\left(176 \mathrm{MHz}, \mathrm{D}_{2} \mathrm{O}\right) \delta 154.10,138.66$, $136.82,119.99,114.35,112.73,52.96$.

Tris((5,6-dimethyl-1H-benzo[d]imidazol-2-yl)methyl)amine, $\left(\mathrm{BimH} / \mathrm{Me}_{2}\right)_{3}$

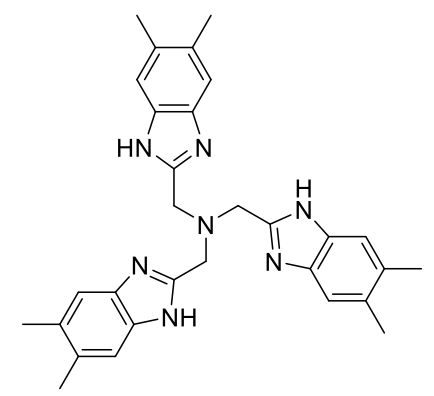

In a $100-\mathrm{mL}$ flask, trinitriloacetic acid $(63.67 \mathrm{mg}, 0.3 \mathrm{mmol}), 4,5$-dimethyl-1,2-phenylenediamine $(136.8 \mathrm{mg}, 1 \mathrm{mmol})$ and $30 \mathrm{~mL}$ of ethylene glycol were added and the mixture was heated at $200{ }^{\circ} \mathrm{C}$ under inert atmosphere for $12 \mathrm{~h}$. After cooling, the product was precipitated in $100 \mathrm{~mL}$ of ice-cooled water. The solid was filtered, washed with $50 \mathrm{~mL}$ of cool water and further purified by silica column using $3 \%$ methanol in DCM as an eluent to afford the product as a white powder. ${ }^{1} \mathrm{H}$ NMR $\left(400 \mathrm{MHz}, \mathrm{THF}-d_{8}\right) \delta 7.33(\mathrm{~s}$, $6 \mathrm{H}), 3.91$ (s, 6H), 2.33 (s, 18H). ${ }^{13} \mathrm{C}$ NMR $\left(101 \mathrm{MHz}, \mathrm{THF}-d_{8}\right) \delta 151.81,138.51,131.22,115.95,52.07,20.44 . \mathrm{MS}(\mathrm{ESI}) \mathrm{m} / z:[\mathrm{M}+$ $\mathrm{H}]^{+}: 492.3$.

Tris((5,6-dichloro-1H-benzo[d]imidazol-2-yl)methyl)amine, $\left(\mathrm{BimH} / \mathrm{Cl}_{2}\right)_{3}$

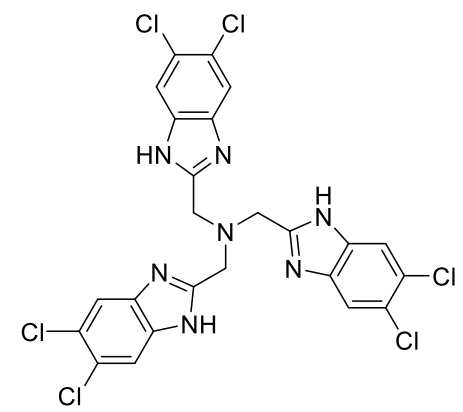

To a solution of 4,5-dichloro-1,2-phenylenediamine ( $88 \mathrm{mg}, 0.5 \mathrm{mmol}$ in $5 \mathrm{~mL}$ of anhydrous dioxane), 2,2,2-nitriloacetyl chloride (41 $\mathrm{mg}, 0.167 \mathrm{mmol}$ in $7 \mathrm{~mL}$ of anhydrous dioxane) was added dropwise and the mixture was refluxed under a nitrogen atmosphere for 7 h. The solvent was then removed under reduced pressure and the residue was taken up in $4 \mathrm{~N} \mathrm{HCl} \mathrm{(20} \mathrm{mL)} \mathrm{and} \mathrm{reflux} \mathrm{for} 6 \mathrm{~h}$. After 
cooling, the acidic solution was basified with concentrated $\mathrm{NH}_{3}$. The precipitate was filtered and washed with $50 \mathrm{~mL}$ of cool water. The product was purified by silica column using 3\% methanol in DCM as eluent. ${ }^{1} \mathrm{H}$ NMR $\left(400 \mathrm{MHz}, \mathrm{THF}-d_{8}\right) \delta 7.73(\mathrm{~s}, 6 \mathrm{H}), 4.13(\mathrm{~s}$, $6 \mathrm{H}) .{ }^{13} \mathrm{C}$ NMR $(101 \mathrm{MHz}, \mathrm{THF}) \delta 155.81,126.36,123.27,117.06,52.62$. HRMS (ESI) calculated for $\left[\mathrm{C}_{24} \mathrm{H}_{16} \mathrm{~N}_{7} \mathrm{Cl}_{6}+\mathrm{H}^{+}\right.$: 611.95983, found: 611.95911.

\section{Tris((5-nitro-1H-benzo[d]imidazol-2-yl)methyl)amine, $\left(\mathrm{BimH} / \mathrm{NO}_{2}\right)_{3}$}

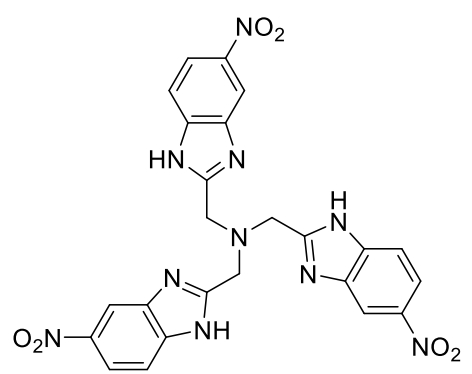

Prepared analogously to $\left(\mathbf{B i m H} / \mathbf{C l}_{2}\right)_{3}$ through the reaction of 4-nitro-1,2-phenylenediamine with 2,2,2-nitriloacetyl chloride. The product was purified by silica column using $3 \%$ methanol in DCM as an eluent. ${ }^{1} \mathrm{H}$ NMR (400 MHz, DMSO- $\left.d_{6}\right) \delta 8.36(\mathrm{~s}, 3 \mathrm{H}), 8.04$ $(\mathrm{d}, J=8.7 \mathrm{~Hz}, 3 \mathrm{H}), 7.68$ (d, $J=8.9 \mathrm{~Hz}, 3 \mathrm{H}), 4.28(\mathrm{~s}, 6 \mathrm{H}) .{ }^{13} \mathrm{C}$ NMR $\left(101 \mathrm{MHz}, \mathrm{DMSO}-d_{6}\right) \delta 151.59,136.19,134.43,132.07,108.77$, 105.90, 103.62, 45.10. HRMS (ESI) calculated for $\left[\mathrm{C}_{24} \mathrm{H}_{19} \mathrm{~N}_{10} \mathrm{O}_{6}+\mathrm{H}\right]^{+}$: 543.14890, found: 543.14786.

\section{$\operatorname{Tris}\left((4,5,6,7-t e t r a f l u o r o-1 H-b e n z o[d]\right.$ imidazol-2-yl)methyl $)$ amine, $\left(\mathrm{BimH} / \mathrm{F}_{4}\right)_{3}$}

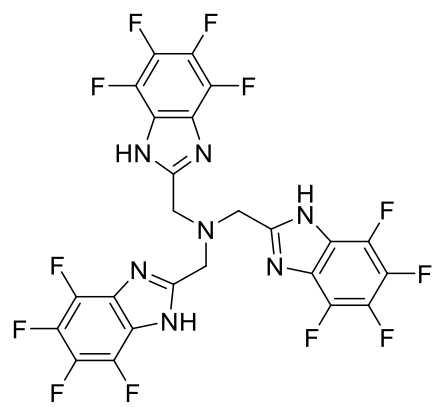

Prepared analogously to $\left(\mathbf{B i m H} / \mathbf{C l}_{2}\right)_{3}$ through the reaction of 3,4,5,6-tetrafluoro-1,2-phenylenediamine with 2,2,2-nitriloacetyl chloride. ${ }^{1} \mathrm{H}$ NMR (400 MHz, Methanol- $\left.d_{4}\right) \delta 4.02(\mathrm{~s}, 6 \mathrm{H}) .{ }^{13} \mathrm{C}$ NMR (101 MHz, Methanol- $\left.d_{4}\right) \delta 158.69,139.10-137.86(\mathrm{~m})$, $136.51-135.27(\mathrm{~m}), 126.83,54.01 .{ }^{19} \mathrm{~F}$ NMR $\left(377 \mathrm{MHz}\right.$, Methanol- $\left.d_{4}\right) \delta-159.65,-170.96 . \mathrm{HRMS}_{(\mathrm{ESI})}$ calculated for $\left[\mathrm{C}_{24} \mathrm{H}_{9} \mathrm{~F}_{12} \mathrm{~N}_{7}+\right.$ $\mathrm{H}]^{+}$: 624.08006, found: 624.08042 . 


\section{Synthesis of $(\mathrm{BimR})_{3}$}
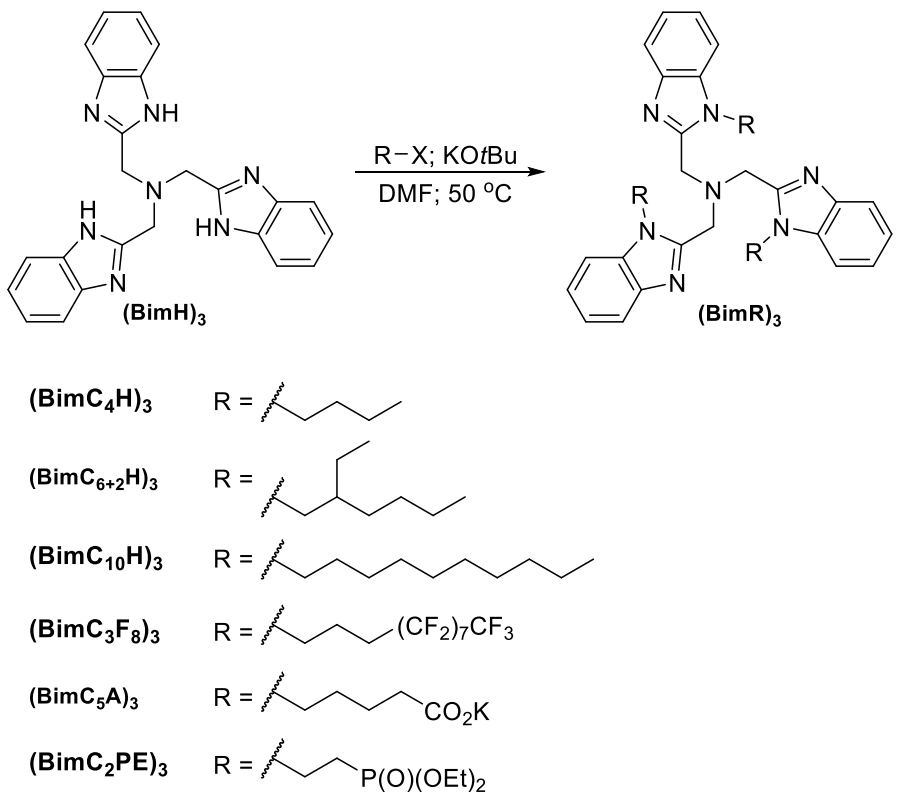

Scheme S2. Synthesis of $(\operatorname{BimR})_{3}$.

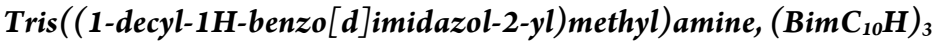

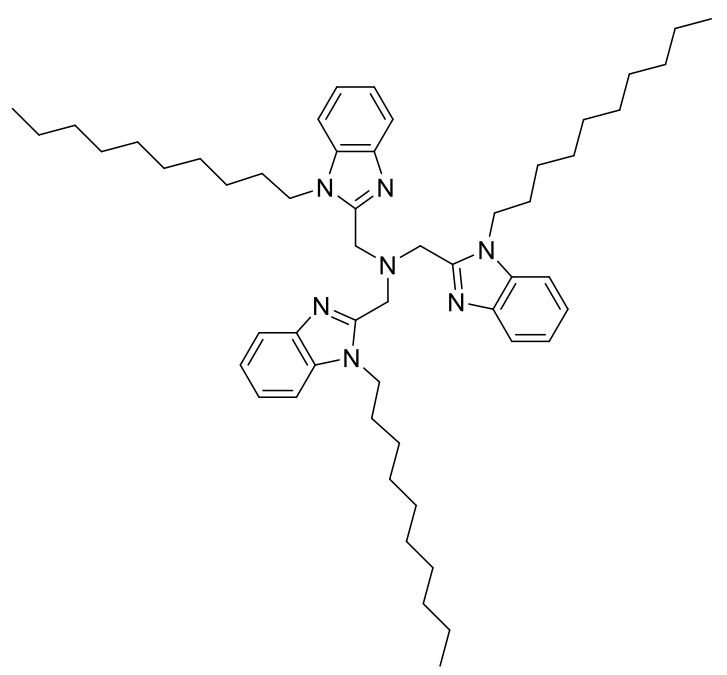

To a $100 \mathrm{~mL}$ schlenk flask protected with argon, $(\mathbf{B i m H})_{3}(1.08 \mathrm{~g}, 2.64 \mathrm{mmol}), t \mathrm{BuOK}(0.98 \mathrm{~g}, 8.7 \mathrm{mmol})$ and $25 \mathrm{~mL}$ of dry DMF were added and the resulting mixture was stirred at $50^{\circ} \mathrm{C}$ for $15 \mathrm{~min}$ followed by the addition of 1 -bromodecane $(1.81 \mathrm{~g}$, $8.2 \mathrm{mmol})$. The mixture was stirred overnight. After cooling, the mixture was transferred to a separatory funnel containing $50 \mathrm{~mL}$ of $\mathrm{H}_{2} \mathrm{O}$ and 50 $\mathrm{mL}$ of DCM. The aqueous layer was extracted twice more with DCM (50 mL) and the combined organic phase was washed with water $(50 \mathrm{~mL} \times 2)$ and then dried over $\mathrm{MgSO}_{4}$. The solvent was then evaporated under reduced pressure and the crude product was purified by silica-gel chromatography using $2.5 \% \mathrm{MeOH}$ in DCM as an eluent. ${ }^{1} \mathrm{H}$ NMR $\left(700 \mathrm{MHz}, \mathrm{DMSO}-d_{6}\right) \delta 7.64(\mathrm{~d}, J=7.7 \mathrm{~Hz}$, $3 \mathrm{H}), 7.42(\mathrm{~d}, J=7.9 \mathrm{~Hz}, 3 \mathrm{H}), 7.24-7.14(\mathrm{~m}, J=14.8,7.2 \mathrm{~Hz}, 6 \mathrm{H}), 4.14(\mathrm{~s}, 6 \mathrm{H}), 3.56(\mathrm{t}, J=7.2 \mathrm{~Hz}, 6 \mathrm{H}), 1.29-1.11(\mathrm{~m}, 24 \mathrm{H}), 1.10-$ 
$1.01(\mathrm{~m}, 12 \mathrm{H}), 0.91-0.82(\mathrm{~m}, 9 \mathrm{H}), 0.81-0.74(\mathrm{~m}, 6 \mathrm{H}), 0.47-0.39(\mathrm{~m}, 6 \mathrm{H}) .{ }^{13} \mathrm{C}$ NMR $\left(176 \mathrm{MHz}, \mathrm{DMSO}-d_{6}\right) \delta 150.60,142.05$, 135.03, 122.39, 121.56, 119.00, 110.20, 49.25, 42.72, 31.27, 29.23, 28.84, 28.66, 28.64, 28.63, 25.64, 22.10, 13.97. HRMS (ESI) calculated for $\left[\mathrm{C}_{54} \mathrm{H}_{81} \mathrm{~N}_{7}+\mathrm{H}\right]^{+}$: 828.66262, found: 828.66100.

Tris((1-(2-ethylhexyl)-1H-benzo[d]imidazol-2-yl)methyl)amine, $\left(\mathrm{BimC}_{6+2} \mathrm{H}\right)_{3}$

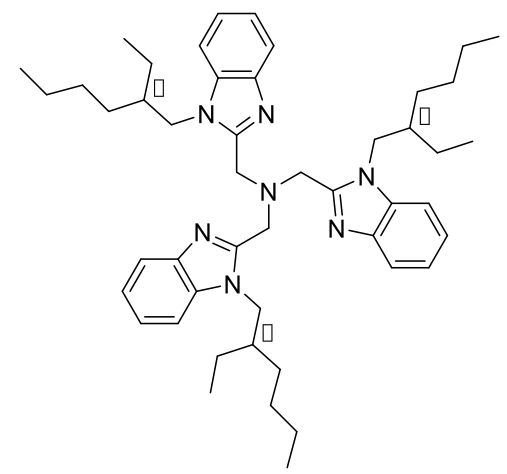

Prepared analogously to $\left(\mathbf{B i m C}_{\mathbf{1 0}} \mathbf{H}\right)_{3}$ by alkylating $(\mathbf{B i m H})_{3}$ with 3-(bromomethyl)heptane. Mixtures of isomers. ${ }^{1} \mathrm{H}$ NMR (700 $\left.\mathrm{MHz}, \mathrm{DMSO}-d_{6}\right) \delta 7.63(\mathrm{~d}, J=7.6 \mathrm{~Hz}, 3 \mathrm{H}), 7.36(\mathrm{~d}, J=4.9 \mathrm{~Hz}, 3 \mathrm{H}), 7.25-7.18(\mathrm{~m}, 6 \mathrm{H}), 4.18-3.97(\mathrm{~m}, 6 \mathrm{H}), 3.47-3.23(\mathrm{~m}, 6 \mathrm{H})$, $1.51-1.42(\mathrm{~m}, 3 \mathrm{H}), 1.00-0.62(\mathrm{~m}, 21 \mathrm{H}), 0.54-0.20(\mathrm{~m}, 21 \mathrm{H}) .{ }^{13} \mathrm{C}$ NMR $\left(176 \mathrm{MHz}, \mathrm{DMSO}-d_{6}\right) \delta 151.11,142.19,135.16,122.81$, $121.95,119.35,110.71,49.57,47.19,38.18,29.56,29.52,29.48,28.15,28.05,27.97,23.14,23.08,23.04,22.44,22.41,22.39$, 13.91, 13.89, 13.86, 10.79, 10.76, 10.72. HRMS (ESI) calculated for $\left[\mathrm{C}_{48} \mathrm{H}_{69} \mathrm{~N}_{7}+\mathrm{H}\right]^{+}:$: 744.56872, found: 744.56801 .

$\operatorname{Tris}\left(\left(1-b u t y l-1 H-b e n z o[d]\right.\right.$ imidazol-2-yl)methyl)amine, $\left(\mathrm{BimC}_{4} \mathrm{H}\right)_{3}$

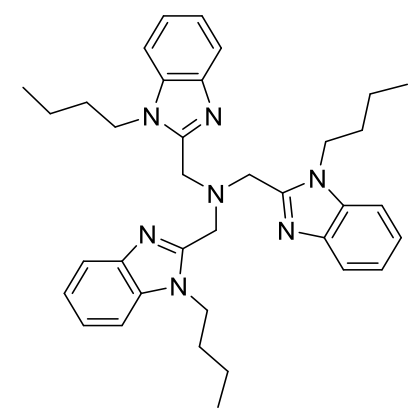

Prepared analogously to $\left(\mathbf{B i m C}_{\mathbf{1 0}} \mathbf{H}\right)_{3}$ by alkylating $(\mathbf{B i m H})_{3}$ with 1-bromobutane. ${ }^{1} \mathrm{H}$ NMR $\left(700 \mathrm{MHz}, \mathrm{DMSO}-d_{6}\right) \delta 7.64(\mathrm{~d}, J=7.7$ $\mathrm{Hz}, 3 \mathrm{H}), 7.45(\mathrm{~d}, J=7.9 \mathrm{~Hz}, 3 \mathrm{H}), 7.24-7.18(\mathrm{~m}, 6 \mathrm{H}), 4.13(\mathrm{~s}, 6 \mathrm{H}), 3.53(\mathrm{t}, J=7.5 \mathrm{~Hz}, 6 \mathrm{H}), 1.06-1.01(\mathrm{~m}, 6 \mathrm{H}), 0.50-0.39(\mathrm{~m}$, $15 \mathrm{H}) .{ }^{13} \mathrm{C}$ NMR $\left(176 \mathrm{MHz}, \mathrm{DMSO}-d_{6}\right) \delta 150.62,142.06,135.10,122.50,121.66,119.03,110.32,49.23,42.47,31.39,18.93,13.53$. MS (ESI) $m / z:[\mathrm{M}+\mathrm{H}]^{+}: 576.2$. 


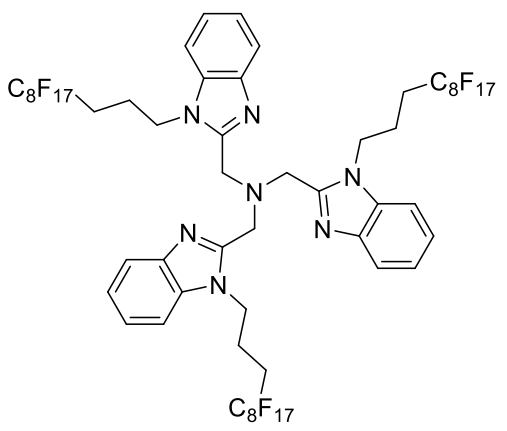

Prepared analogously to $\left(\mathbf{B i m C}_{\mathbf{1 0}} \mathbf{H}\right)_{3}$ by alkylating $(\mathbf{B i m H})_{3}$ with $1 H, 1 H, 2 H, 2 H, 3 H, 3 H$-perfluoroundecyl iodide. Purified by recrystallization from a mixed solvent of ethyl acetate and hexane. ${ }^{1} \mathrm{H}$ NMR $\left(700 \mathrm{MHz}\right.$, Acetone- $\left.d_{6}\right) \delta 7.71(\mathrm{~d}, J=7.6 \mathrm{~Hz}, 3 \mathrm{H}), 7.51(\mathrm{~d}$, $J=7.9 \mathrm{~Hz}, 3 \mathrm{H}), 7.29-7.22(\mathrm{~m}, 6 \mathrm{H}), 4.38(\mathrm{~s}, 6 \mathrm{H}), 3.95(\mathrm{t}, J=7.2 \mathrm{~Hz}, 6 \mathrm{H}), 1.82-1.69(\mathrm{~m}, 12 \mathrm{H}) .{ }^{13} \mathrm{C} \mathrm{NMR}\left(176 \mathrm{MHz}\right.$, Acetone- $\left.d_{6}\right) \delta$ $152.11,143.57,136.14,123.73,122.92,120.35,110.76,50.39,42.80,28.10(\mathrm{t}, J=22.1 \mathrm{~Hz}), 21.45 .{ }^{19} \mathrm{~F} \mathrm{NMR}\left(377 \mathrm{MHz}\right.$, Acetone- $\left.d_{6}\right) \delta$ -81.70, -114.58, -122.38, -122.51, -123.33, -123.62, -126.79. MS (ESI) $m / z:[\mathrm{M}+\mathrm{H}]^{+}$: 1787.9; HRMS (ESI) calculated for $\left[\mathrm{C}_{57} \mathrm{H}_{36} \mathrm{~F}_{51} \mathrm{~N}_{7}+\mathrm{H}\right]^{+}:$1788.22906, found: 1788.22392 . 
Synthesis of $\left(\operatorname{BimR}^{1}\right)_{2}\left(\operatorname{BimR}^{2}\right)$

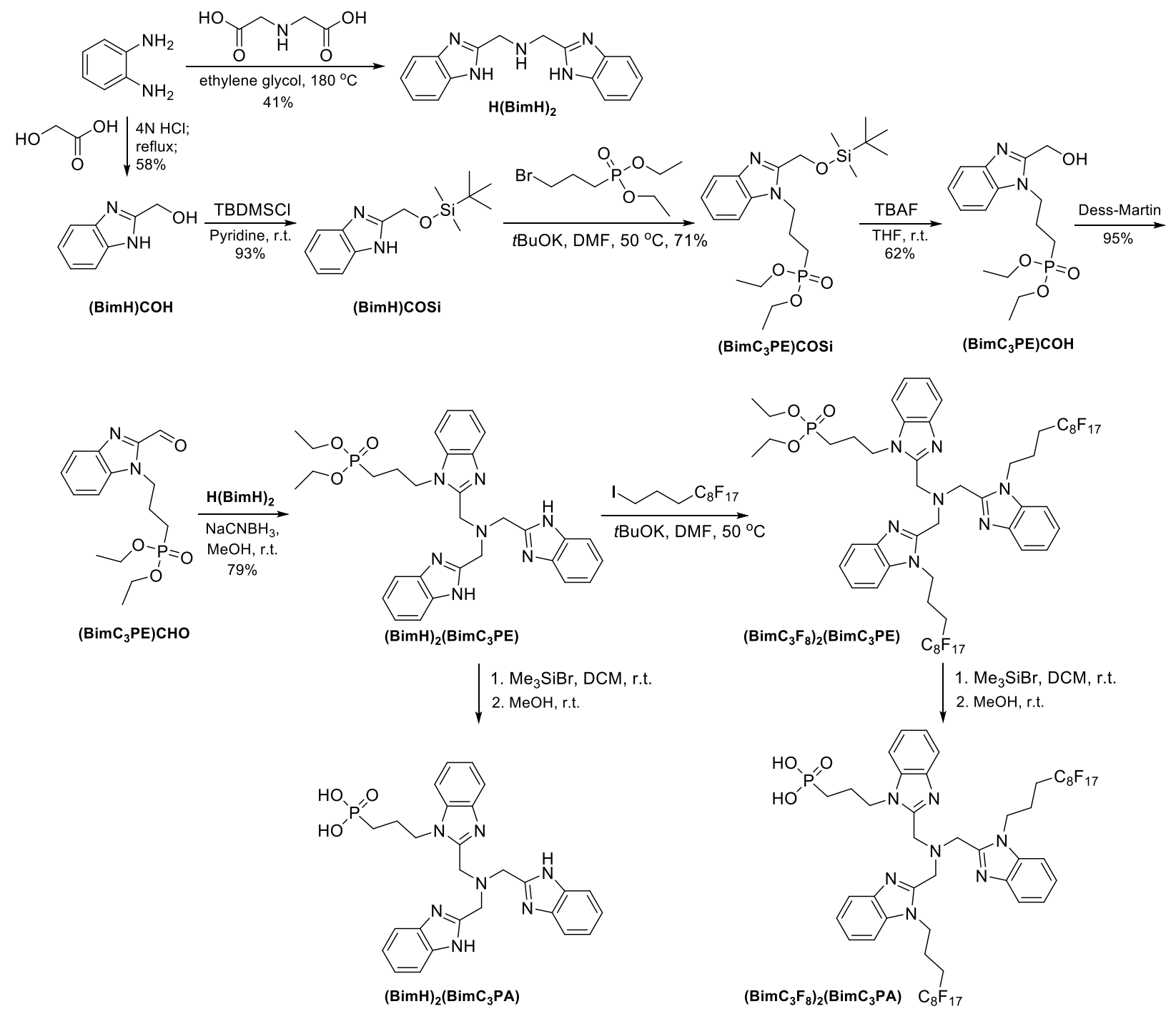

Scheme S3. Synthesis of $\left(\operatorname{BimR}^{1}\right)_{2}\left(\operatorname{BimR}^{2}\right)$.

\section{(1H-benzo[d]imidazol-2-yl)methanol, (BimH)COH}

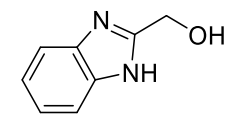

Prepared following a previously reported procedure. ${ }^{9}$ White solid. ${ }^{1} \mathrm{H}$ NMR (400 MHz, Methanol- $\left.\mathrm{d}_{4}\right) \delta 7.56-7.50(\mathrm{~m}, 2 \mathrm{H}), 7.24-$ $7.18(\mathrm{~m}, 2 \mathrm{H}), 4.83(\mathrm{~s}, 2 \mathrm{H}) .{ }^{13} \mathrm{C}$ NMR $\left(126 \mathrm{MHz}\right.$, Methanol-d $\left.\mathrm{d}_{4}\right) \delta 156.18$, 123.41, 58.93. MS (ESI) m/z: $[\mathrm{M}+\mathrm{H}]^{+}: 149.1$. 


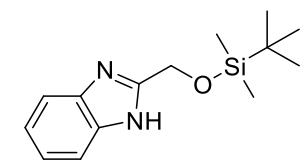

Prepared following a previously reported procedure. ${ }^{10}$ White solid. ${ }^{1} \mathrm{H}$ NMR (500 MHz, Acetone- $\left.\mathrm{d}_{6}\right) \delta 7.62-7.43(\mathrm{~m}, 2 \mathrm{H}), 7.16(\mathrm{~d}, J$ $=6.4 \mathrm{~Hz}, 2 \mathrm{H}), 4.98$ (s, 2H), 0.93 (s, 9H), 0.15 (s, 6H). ${ }^{13} \mathrm{C}$ NMR (126 MHz, Acetone-d 6 ) $\delta 122.85,122.12,119.74,111.95,60.74$, 26.19, 18.90, -5.32. MS (ESI) m/z: $[\mathrm{M}+\mathrm{H}]^{+}: 263.1$.

Diethyl (3-(2-((tert-butyldimethylsilyl)oxy)methyl)-1H-benzo[d]imidazol-1-yl)propyl)phosphonate, (BimC $\left.{ }_{3} P E\right) C O S i$

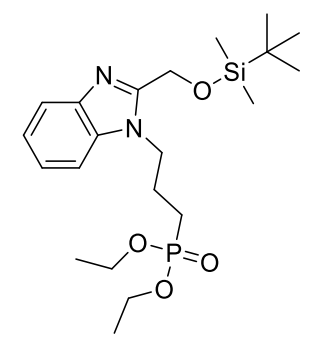

To a $100 \mathrm{~mL}$ schlenk flask protected with argon, (BimH)COSi $(0.95 \mathrm{~g}, 3.45 \mathrm{mmol}), t \mathrm{BuOK}(0.46 \mathrm{~g}, 4.14 \mathrm{mmol})$ and $40 \mathrm{~mL}$ of dry DMF were added and the resulting mixture was stirred at $50{ }^{\circ} \mathrm{C}$ for $15 \mathrm{~min}$ followed by addition of diethyl (3bromopropyl)phosphonate $(1.07 \mathrm{~g}, 4.14 \mathrm{mmol})$. The mixture was stirred overnight. After cooling, the mixture was transferred to a separatory funnel containing $100 \mathrm{~mL}$ of $\mathrm{H}_{2} \mathrm{O}$ and $70 \mathrm{~mL}$ of DCM. The aqueous layer was extracted twice more with $\mathrm{DCM}$ (50 mL) and the combined organic phase was washed with water $(70 \mathrm{~mL} \times 2)$, and then dried over $\mathrm{MgSO}_{4}$. The solvent was then evaporated under reduced pressure and the crude product was purified by silica-gel chromatography using a mixed solvent of ethyl acetate and petroleum ether (3:4) as an eluent. White solid. Yield: 71\%. ${ }^{1} \mathrm{H}$ NMR (400 MHz, Chloroform-d) $\delta 7.77-7.72(\mathrm{~m}, 1 \mathrm{H}), 7.44-7.40$ $(\mathrm{m}, 1 \mathrm{H}), 7.31-7.22(\mathrm{~m}, 2 \mathrm{H}), 4.97(\mathrm{~s}, 2 \mathrm{H}), 4.38(\mathrm{t}, J=7.5 \mathrm{~Hz}, 2 \mathrm{H}), 4.15-4.01(\mathrm{~m}, 4 \mathrm{H}), 2.26-2.11(\mathrm{~m}, 2 \mathrm{H}), 1.84-1.71(\mathrm{~m}, 2 \mathrm{H})$, $1.30(\mathrm{t}, J=7.1 \mathrm{~Hz}, 6 \mathrm{H}), 0.90(\mathrm{~s}, 9 \mathrm{H}), 0.11(\mathrm{~s}, 6 \mathrm{H}) .{ }^{13} \mathrm{C}$ NMR $(101 \mathrm{MHz}$, Chloroform-d) $\delta 152.29,142.35,135.63,123.10,122.26$, $120.24,109.79,61.88(\mathrm{~d}, J=6.6 \mathrm{~Hz}), 59.66,44.24(\mathrm{~d}, J=16.9 \mathrm{~Hz}), 26.00,23.32(\mathrm{~d}, J=143.2 \mathrm{~Hz}), 23.20(\mathrm{~d}, J=4.9 \mathrm{~Hz}), 18.45$, $16.62(\mathrm{~d}, J=5.9 \mathrm{~Hz}),-5.22 .{ }^{31} \mathrm{P}$ NMR $(162 \mathrm{MHz}$, Chloroform- $d) \delta 30.49$. HRMS (ESI) calculated for $\left[\mathrm{C}_{21} \mathrm{H}_{37} \mathrm{~N}_{2} \mathrm{O}_{4} \mathrm{PSi}+\mathrm{H}\right]^{+}$: 441.23330, found: 441.23213 .

Diethyl (3-(2-(hydroxymethyl)-1H-benzo[d]imidazol-1-yl)propyl)phosphonate, $\left(\right.$ Bim $\left.\mathrm{C}_{3} \mathrm{PE}\right) \mathrm{COH}$

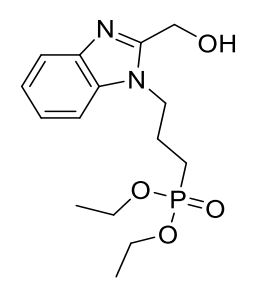

In a $10 \mathrm{~mL}$ flask, $\left(\mathbf{B i m C}_{3} \mathbf{P E}\right) \mathbf{C O S i}(0.68 \mathrm{~g}, 1.54 \mathrm{mmol})$ and TBAF (1 M in THF, $\left.3.2 \mathrm{~mL}\right)$ were stirred at room temperature overnight. The solution was evaporated to dryness, dissolved in $100 \mathrm{~mL}$ ethyl acetate, washed with brine $(20 \mathrm{~mL} \cdot 3)$ and dried over $\mathrm{MgSO}_{4}$. The solvent was then evaporated under reduced pressure and the product was obtained as a colorless oil. Yield: $62 \%$. ${ }^{1} \mathrm{H} \mathrm{NMR}(500 \mathrm{MHz}$, 
Chloroform-d) $\delta 7.65-7.61(\mathrm{~m}, 1 \mathrm{H}), 7.34-7.30(\mathrm{~m}, 1 \mathrm{H}), 7.24-7.16(\mathrm{~m}, 2 \mathrm{H}), 5.36(\mathrm{br}, 1 \mathrm{H}), 4.85(\mathrm{~s}, 2 \mathrm{H}), 4.30(\mathrm{t}, J=7.5 \mathrm{~Hz}, 2 \mathrm{H})$, $4.08-3.97(\mathrm{~m}, 4 \mathrm{H}), 2.19-2.06(\mathrm{~m}, 2 \mathrm{H}), 1.81-1.70(\mathrm{~m}, 2 \mathrm{H}), 1.24(\mathrm{t}, J=7.1 \mathrm{~Hz}, 6 \mathrm{H}) .{ }^{13} \mathrm{C} \mathrm{NMR}(126 \mathrm{MHz}, \mathrm{Chloroform}-d) \delta 153.79$ (s), 141.39 (s), 134.93 (s), 123.10 (s), 122.43 (s), 119.36 (s), 109.78 (s), 61.93 (d, J = 6.5 Hz), 56.74 (s), 43.80 (d, J = 15.3 Hz), 22.95 $(\mathrm{d}, J=4.8 \mathrm{~Hz}), 22.64(\mathrm{~d}, J=142.7 \mathrm{~Hz}), 16.44(\mathrm{~d}, J=5.9 \mathrm{~Hz}) .{ }^{31} \mathrm{P} \mathrm{NMR}(243 \mathrm{MHz}$, Chloroform- $d) \delta 30.72 . \mathrm{MS}(\mathrm{ESI}) m / z:[\mathrm{M}+\mathrm{H}]^{+}$: 327.0; HRMS (ESI) calculated for $\left[\mathrm{C}_{15} \mathrm{H}_{23} \mathrm{~N}_{2} \mathrm{O}_{4} \mathrm{P}+\mathrm{H}\right]^{+}: 327.14682$, found: 327.14613 .

\section{Diethyl (3-(2-formyl-1H-benzo[d]imidazol-1-yl)propyl)phosphonate, (BimC ${ }_{3}$ PE)CHO}

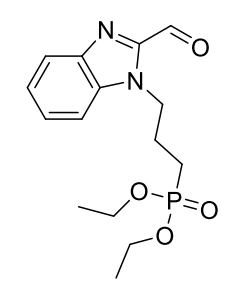

To a $25 \mathrm{~mL}$ flask, $\left(\mathbf{B i m C}_{3} \mathbf{P E}\right) \mathbf{C O H}(280 \mathrm{mg}, 0.86 \mathrm{mmol})$ and $5 \mathrm{~mL}$ of dichloromethane were added and the mixture was put in an ice bath with Dess-Martin periodinane $(424 \mathrm{mg}, 1 \mathrm{mmol})$. After $2 \mathrm{~h}$ of stirring, the solution was diluted by adding $50 \mathrm{~mL}$ of dichloromethane and washed with saturated $\mathrm{Na}_{2} \mathrm{~S}_{2} \mathrm{O}_{3}$ and $\mathrm{NaHCO}_{3}$ aqueous solution separately. The organic phase was then evaporated under reduced pressure and the product was obtained as a colorless oil. Yield: $95 \% .{ }^{1} \mathrm{H} \mathrm{NMR}(500 \mathrm{MHz}$, Chloroform- $d$ ) $\delta$ $10.03(\mathrm{~s}, 1 \mathrm{H}), 7.85(\mathrm{~d}, J=8.3 \mathrm{~Hz}, 1 \mathrm{H}), 7.51(\mathrm{~d}, J=8.3 \mathrm{~Hz}, 1 \mathrm{H}), 7.44-7.40(\mathrm{~m}, 1 \mathrm{H}), 7.35-7.31(\mathrm{~m}, 1 \mathrm{H}), 4.68-4.59(\mathrm{~m}, 2 \mathrm{H}), 4.09-$ $3.95(\mathrm{~m}, 4 \mathrm{H}), 2.15-2.03(\mathrm{~m}, 2 \mathrm{H}), 1.76-1.65(\mathrm{~m}, 2 \mathrm{H}), 1.23(\mathrm{t}, J=7.1 \mathrm{~Hz}, 6 \mathrm{H}) .{ }^{13} \mathrm{C}$ NMR $(126 \mathrm{MHz}, \mathrm{Chloroform}-d) \delta 184.85$ $145.73,142.71,136.27,127.09$, 124.23 , 122.39 , 110.95 , 61.75 (d, $J=6.8 \mathrm{~Hz}), 44.57$ (d, J = 16.4 Hz), 23.51 (d, J = 4.8 Hz), $22.67(\mathrm{~d}$, $J=143.1 \mathrm{~Hz}), 16.42(\mathrm{~d}, J=5.9 \mathrm{~Hz}) .{ }^{31} \mathrm{P} \mathrm{NMR}(243 \mathrm{MHz}$, Chloroform-d $) \delta 30.40$. HRMS (ESI) calculated for $\left[\mathrm{C}_{15} \mathrm{H}_{21} \mathrm{~N}_{2} \mathrm{O}_{4} \mathrm{P}+\mathrm{H}\right]^{+}$: 325.13117, found: 325.13039 .

\section{Bis $\left(\left(1 \mathrm{H}-\right.\right.$ benzo $[\mathrm{d}]$ imidazol-2-yl)methyl)amine, $\mathrm{H}(\mathrm{BimH})_{2}$}

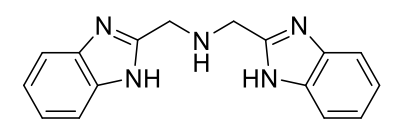

Prepared following a previously reported procedure. ${ }^{11}$ White solid. ${ }^{1} \mathrm{H}$ NMR $\left(700 \mathrm{MHz}\right.$, Methanol- $\left.d_{4}\right) \delta 7.59-7.48(\mathrm{~m}, 4 \mathrm{H}), 7.28-$ $7.16(\mathrm{~m}, 4 \mathrm{H}), 4.11(\mathrm{~s}, 4 \mathrm{H}) \cdot{ }^{13} \mathrm{C}$ NMR $\left(176 \mathrm{MHz}\right.$, Methanol- $\left.d_{4}\right) \delta 155.03,123.51,47.40 . \mathrm{MS}(\mathrm{ESI}) \mathrm{m} / z:[\mathrm{M}+\mathrm{H}]^{+}: 278.1$. 
$(\mathrm{BimH})_{2}\left(\mathrm{BimC}_{3} \mathrm{PE}\right)$

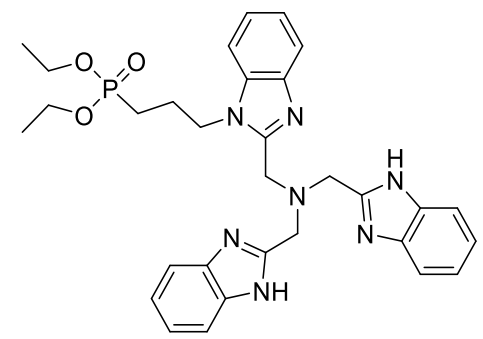

$\mathbf{H}(\mathbf{B i m H})_{2}(302 \mathrm{mg}, 1.09 \mathrm{mmol})$ and $\left(\mathbf{B i m C}_{3} \mathbf{P E}\right) \mathbf{C H O}(0.338 \mathrm{~g}, 1.04 \mathrm{mmol})$ were dissolved in dry methanol $(10 \mathrm{~mL})$. The solution was cooled to $0{ }^{\circ} \mathrm{C}$ and $\mathrm{SOCl}_{2}(25 \mu \mathrm{L})$ was added followed by $\mathrm{NaBH}_{3} \mathrm{CN}(342 \mathrm{mg}, 5.45 \mathrm{mmol})$. The reaction mixture was stirred under $\mathrm{N}_{2}$ for $10 \mathrm{~h}$. The solution was evaporated to dryness and $50 \mathrm{~mL}$ of water was added. The aqueous solution was then extracted with $\mathrm{CH}_{2} \mathrm{Cl}_{2}(50 \mathrm{~mL} \cdot 3)$ and the combined organic fractions were washed once with water $(50 \mathrm{~mL})$, and then dried over $\mathrm{MgSO}_{4}$. The solvent was removed by rotary evaporation and the crude product was purified by silica-gel chromatography, using $2 \% \mathrm{MeOH}$ in DCM as eluent. ${ }^{1} \mathrm{H}$ NMR $\left(700 \mathrm{MHz}, \mathrm{DMSO}-d_{6}\right) \delta 12.43(\mathrm{br}, 2 \mathrm{H}), 7.69-7.44(\mathrm{~m}, 6 \mathrm{H}), 7.27-7.12(\mathrm{~m}, 6 \mathrm{H}), 4.33-4.29(\mathrm{~m}, 2 \mathrm{H})$, $4.20(\mathrm{~s}, 2 \mathrm{H}), 4.10(\mathrm{~s}, 4 \mathrm{H}), 3.87-3.81(\mathrm{~m}, 4 \mathrm{H}), 1.83-1.74(\mathrm{~m}, 2 \mathrm{H}), 1.60-1.52(\mathrm{~m}, 2 \mathrm{H}), 1.12(\mathrm{t}, J=7.1 \mathrm{~Hz}, 6 \mathrm{H}) .{ }^{13} \mathrm{C}$ NMR $(176$ MHz, DMSO-d 6 ) $\delta 151.68,151.04,143.17,142.01$, 135.39 , 134.20 , 122.30 , 122.05 , 121.55 , 121.17 , 119.09 , 118.59 , 111.30 , $110.30,61.00(\mathrm{~d}, J=6.2 \mathrm{~Hz}), 51.14,50.03,43.05(\mathrm{~d}, J=18.1 \mathrm{~Hz}), 22.70(\mathrm{~d}, J=4.0 \mathrm{~Hz}), 21.49(\mathrm{~d}, J=139.5 \mathrm{~Hz}), 16.24(\mathrm{~d}, J=5.7$ $\mathrm{Hz}) .{ }^{31} \mathrm{P}$ NMR $\left(243 \mathrm{MHz}\right.$, DMSO- $\left.d_{6}\right) \delta$ 31.03. HRMS (ESI) calculated for $\left[\mathrm{C}_{31} \mathrm{H}_{36} \mathrm{~N}_{7} \mathrm{O}_{3} \mathrm{P}+\mathrm{H}\right]^{+}: 586.26900$, found: 586.26768 .

Diethyl (3-(2-((bis $((1-(1 \mathrm{H}, 1 \mathrm{H}, 2 \mathrm{H}, 2 \mathrm{H}, 3 \mathrm{H}, 3 \mathrm{H}-\mathrm{perfluoroundecyl})-1 \mathrm{H}-$ benzo[d]imidazol-2-yl $)$ methyl $)$ amino $)$ methyl)-1Hbenzo $[d]$ imidazol-1-yl)propyl)phosphonate, $\left(\mathrm{BimC}_{3} \mathrm{~F}_{8}\right)_{2}\left(\mathrm{BimC}_{3} \mathrm{PE}\right)$

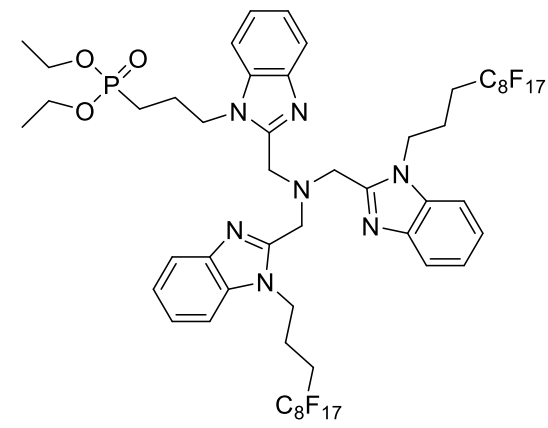

Prepared analogously to $\left(\mathbf{B i m C}_{3} \mathbf{F}_{8}\right)_{3}$ by alkylating $(\mathbf{B i m H})_{2}\left(\mathbf{B i m C}_{3} \mathbf{P E}\right)$ with $1 \mathrm{H}, 1 \mathrm{H}, 2 \mathrm{H}, 2 \mathrm{H}, 3 \mathrm{H}, 3 \mathrm{H}$-perfluoroundecyl iodide. ${ }^{1} \mathrm{H}$ $\operatorname{NMR}\left(700 \mathrm{MHz}\right.$, Methanol- $\left.d_{4}\right) \delta 7.75-7.69(\mathrm{~m}, 3 \mathrm{H}), 7.49(\mathrm{~d}, J=7.6 \mathrm{~Hz}, 1 \mathrm{H}), 7.46(\mathrm{~d}, J=7.6 \mathrm{~Hz}, 2 \mathrm{H}), 7.37-7.28(\mathrm{~m}, 6 \mathrm{H}), 4.24(\mathrm{~s}$, 4H), $4.22(\mathrm{~s}, 2 \mathrm{H}), 3.97-3.91(\mathrm{~m}, 4 \mathrm{H}), 3.65(\mathrm{t}, J=7.5 \mathrm{~Hz}, 4 \mathrm{H}), 3.57(\mathrm{t}, J=7.6 \mathrm{~Hz}, 2 \mathrm{H}), 1.55-1.38(\mathrm{~m}, 10 \mathrm{H}), 1.24(\mathrm{t}, J=7.1 \mathrm{~Hz}$, $6 \mathrm{H}), 0.92-0.85(\mathrm{~m}, 2 \mathrm{H}) .{ }^{13} \mathrm{C}$ NMR $\left(176 \mathrm{MHz}\right.$, Methanol- $\left.d_{4}\right) \delta 151.93,151.90,142.65$, $142.55,136.06$, $136.04,125.15,125.03$, $124.37,124.27,120.28,120.09,111.53,111.40,63.30(\mathrm{~d}, J=6.6 \mathrm{~Hz}), 50.53,50.47,44.16(\mathrm{~d}, J=17.9 \mathrm{~Hz}), 43.14,28.26(\mathrm{t}, J=22.2$ $\mathrm{Hz}), 23.59(\mathrm{~d}, J=4.4 \mathrm{~Hz}), 22.18(\mathrm{~d}, J=142.8 \mathrm{~Hz}), 21.58,16.70(\mathrm{~d}, J=5.9 \mathrm{~Hz}) .{ }^{31} \mathrm{P}$ NMR $\left(243 \mathrm{MHz}\right.$, Methanol- $\left.d_{4}\right) \delta 30.99 .{ }^{19} \mathrm{~F}$ NMR $\left(377 \mathrm{MHz}\right.$, Methanol- $\left.d_{4}\right) \delta$-82.39, -115.08, -122.80, -122.94, -123.77, -123.92, -127.32. MS (ESI) $m / z:[\mathrm{M}+\mathrm{H}]^{+}: 1506.2$; HRMS (ESI) calculated for $\left[\mathrm{C}_{53} \mathrm{H}_{46} \mathrm{~F}_{34} \mathrm{~N}_{7} \mathrm{O}_{3} \mathrm{P}+\mathrm{H}\right]^{+}:$1506.29296, found: 1506.28940 . 


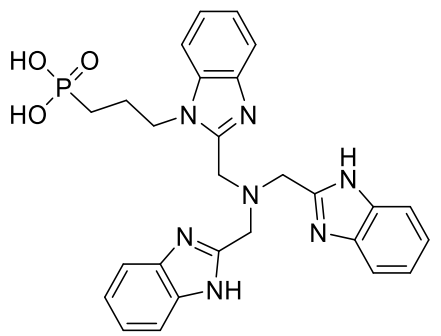

To a flask under argon atmosphere, $(\mathbf{B i m H})_{2}\left(\mathbf{B i m C}_{3} \mathbf{P E}\right)(41 \mathrm{mg}, 0.07 \mathrm{mmol})$ and DCM $(10 \mathrm{~mL})$ were added. Then $\mathrm{SiMe} \mathrm{Br}(86$ $\mathrm{mg}, 0.56 \mathrm{mmol}$ ) was slowly added and the mixture was stirred at room temperature for $2 \mathrm{~d}$. The solvents were evaporated under reduced pressure; $\mathrm{MeOH}(20 \mathrm{~mL})$ was added and stirred for $2 \mathrm{~h}$ and then evaporated under reduced pressure. The product was dried under a vacuum to yield $(\mathbf{B i m H})_{2}\left(\mathbf{B i m C}_{3} \mathbf{P A}\right)$ with sufficient purity. ${ }^{1} \mathrm{H}$ NMR $\left(700 \mathrm{MHz}\right.$, Methanol- $\left.d_{4}\right) \delta 8.00-7.88(\mathrm{~m}, 2 \mathrm{H}), 7.85-$ $7.80(\mathrm{~m}, 4 \mathrm{H}), 7.65-7.57(\mathrm{~m}, 6 \mathrm{H}), 4.93-4.73(\mathrm{~m}, 8 \mathrm{H}), 2.31-2.24(\mathrm{~m}, 2 \mathrm{H}), 2.06-1.99(\mathrm{~m}, 2 \mathrm{H}) .{ }^{13} \mathrm{C}$ NMR $(176 \mathrm{MHz}, \mathrm{Methanol}$ $\left.d_{4}\right) \delta 148.85,148.27,132.52,131.08,130.53,126.85,126.60,126.49,114.27,113.76,112.65,50.62,50.10,45.46(\mathrm{~d}, \mathrm{~J}=10.0$ $\mathrm{Hz}$ ), $23.22(\mathrm{~d}, J=138.4 \mathrm{~Hz}), 22.92(\mathrm{~d}, J=3.8 \mathrm{~Hz}) \cdot{ }^{31} \mathrm{P}$ NMR $\left(243 \mathrm{MHz}\right.$, Methanol- $\left.d_{4}\right) \delta$ 27.45. HRMS (ESI) calculated for $\left[\mathrm{C}_{27} \mathrm{H}_{28} \mathrm{~N}_{7} \mathrm{O}_{3} \mathrm{P}+\mathrm{H}\right]^{+}$: 530.20640, found: 530.20528 .

(3-(2-((Bis $((1-(1 H, 1 H, 2 H, 2 H, 3 H, 3 H-p e r f l u o r o u n d e c y l)-1 H-b e n z o[d]$ imidazol-2-yl $)$ methyl $)$ amino $)$ methyl)-1Hbenzo $[d]$ imidazol-1-yl) propyl)phosphonic acid, $\left(\mathrm{BimC}_{3} \mathrm{~F}_{8}\right)_{2}\left(\mathrm{BimC}_{3} \mathrm{PA}\right)$

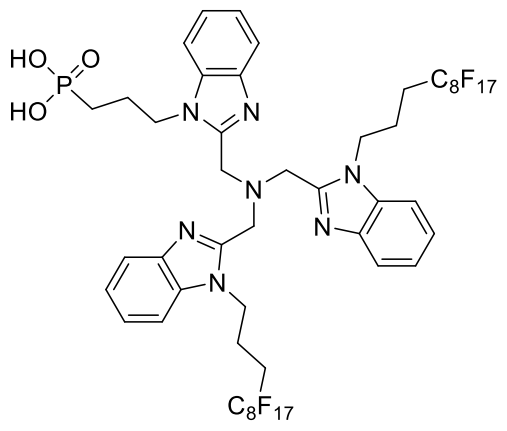

Prepared analogously to $(\mathbf{B i m H})_{2}\left(\mathbf{B i m C}_{3} \mathbf{P A}\right)$ by treating $\left(\mathbf{B i m C}_{3} \mathbf{F}_{\mathbf{8}}\right)_{\mathbf{2}}\left(\mathbf{B i m C}_{\mathbf{3}} \mathbf{P E}\right)$ with $\mathrm{SiMe}_{3} \mathrm{Br} .{ }^{1} \mathrm{H} \mathrm{NMR}\left(700 \mathrm{MHz}, \mathrm{Methanol}-d_{4}\right)$ $\delta 8.04-7.91(\mathrm{~m}, 6 \mathrm{H}), 7.72-7.59(\mathrm{~m}, 6 \mathrm{H}), 5.09(\mathrm{~s}, 4 \mathrm{H}), 5.03(\mathrm{~s}, 2 \mathrm{H}), 4.82-4.73(\mathrm{~m}, 6 \mathrm{H}), 2.64-2.51(\mathrm{~m}, 4 \mathrm{H}), 2.34-2.17(\mathrm{~m}, 6 \mathrm{H})$, 2.08 - 1.99 (m, 2H). ${ }^{31} \mathrm{P}$ NMR (162 MHz, Methanol- $\left.d_{4}\right) \delta$ 27.96. ${ }^{19} \mathrm{~F}$ NMR (377 MHz, Methanol- $\left.d_{4}\right) \delta-82.40,-115.18,-122.67,-$ 122.93, -123.78, -124.28, -127.33. HRMS (ESI) calculated for $\left[\mathrm{C}_{49} \mathrm{H}_{38} \mathrm{~F}_{34} \mathrm{~N}_{7} \mathrm{O}_{3} \mathrm{P}+\mathrm{H}\right]^{+}$: 1450.23036, found: 1450.22631 . 


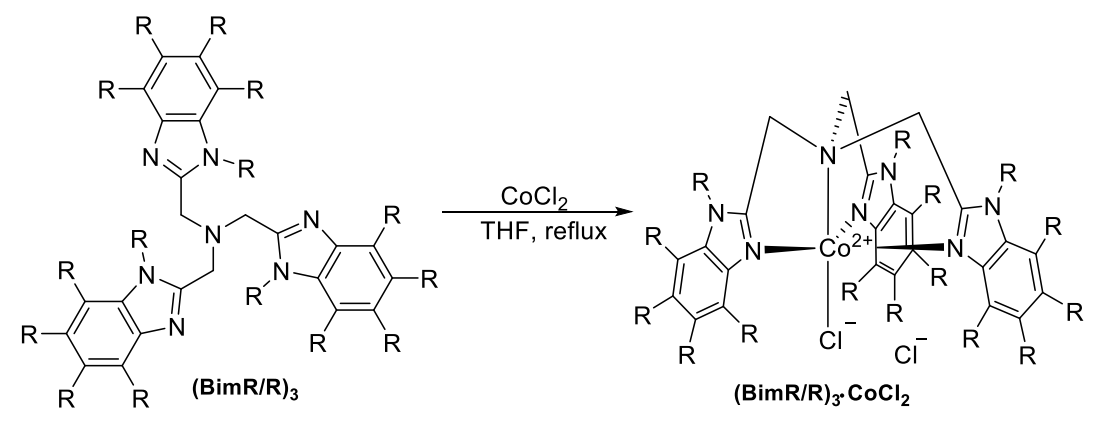

Scheme S4. Synthesis of Co(II) complexes.

\section{$\operatorname{Co}-\left(\operatorname{BimC}_{3} \mathrm{~F}_{8}\right)_{3}$}

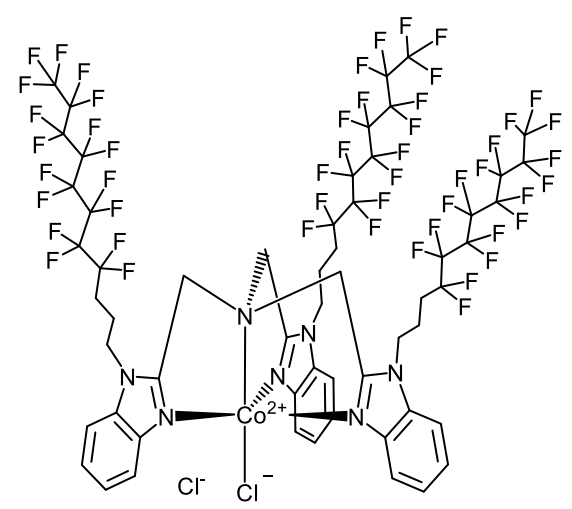

To a $50 \mathrm{~mL}$ flask, $\left(\mathbf{B i m C}_{3} \mathbf{F}_{8}\right)_{3}(179 \mathrm{mg}, 0.1 \mathrm{mmol})$, anhydrous $\mathrm{CoCl}_{2}(14 \mathrm{mg}, 0.11 \mathrm{mmol})$ and $20 \mathrm{~mL}$ of THF were added and the resulting solution was stirred and refluxed for $2 \mathrm{~h}$. After cooling, the solvent was evaporated under reduced pressure and the resulting solid was washed with water $(5 \mathrm{ml} \times 3)$ to afford the product as a purple solid. $\mathrm{MS}(\mathrm{ESI}) \mathrm{m} / \mathrm{z}$ : $\left[\left(\mathbf{B i m C}_{3} \mathbf{F}_{8}\right)_{3}+\mathrm{Co}^{2+}+\mathrm{CF}_{3} \mathrm{COO}^{-}\right.$ ]$^{+}: 1958.8 ;\left[\left(\mathbf{B i m C}_{3} \mathrm{~F}_{8}\right)_{3}+\mathrm{Co}^{2+}+\mathrm{Cl}^{-}\right]^{+}:$1881.8. $\left(0.1 \% \mathrm{CF}_{3} \mathrm{COOH}\right.$ was added in the eluent of LCMS $)$ Calcd. for $\mathrm{C}_{57} \mathrm{H}_{42} \mathrm{Cl}_{2} \mathrm{CoF}_{51} \mathrm{~N}_{7} \mathrm{O}_{3}$ $\left(\mathbf{C o}\left(\mathbf{B i m C}_{3} \mathbf{F}_{8}\right)_{3} \cdot 3 \mathrm{H}_{2} \mathrm{O}\right):$ C, 34.72; H, 2.15; N, 4.97. Found: C, 34.57; H, 2.38; N, 5.11. The single crystal X-ray structure of Co$\left(\mathbf{B i m C}_{3} \mathbf{F}_{8}\right)_{3}$ was also obtained (for details, see Section 8). The other complexes were prepared analogously to Co- $\left(\mathbf{B i m C}_{3} \mathbf{F}_{8}\right)_{3}$ by refluxing the ligands with anhydrous $\mathrm{CoCl}_{2}$ in THF. 


\section{Preparation of Working Electrodes}

We selected fluorine-doped tin-oxide (FTO) coated glass substrates to prepare catalyst-functionalized electrodes. The FTO conductive layer is transparent, oxidatively stable, and tolerant to mechanical abrasion. FTO glass ( $15 \mathrm{ohm} / \mathrm{sq}$, Hartford Glass) substrates (size: $1 \mathrm{~cm} \times 3 \mathrm{~cm}$ ) were cleaned by consecutive 30-min sonication in detergent, deionized water, acetone and ethanol. After cleaning, the electrodes were dried at $120^{\circ} \mathrm{C}$ for at least $1 \mathrm{~h}$.

To prepare the working electrodes, planar FTO substrates were loaded with the molecular species by drop casting. A drop of a solution with a known concentration was deposited onto the surface of the electrode covering $1 \mathrm{~cm}^{2}$. A $10^{-3} \mathrm{M}$ solution of Co-complex in methanol was used to prepare stock solutions (total volume, $1 \mathrm{ml}$ ). Then, $10 \mu \mathrm{l}$ of the stock solution was deposited onto the FTO electrodes to obtain different loadings $0.5-2 \times 10^{-9} \mathrm{~mol} \cdot \mathrm{cm}^{-2}$.

Table S3. Contact angle measurement on silicon wafer slides modified with $5 \times 10^{-10} \mathrm{~mol} \cdot \mathrm{cm}^{-2}$ of Co-trisbenzimidazoles.

\begin{tabular}{ccc}
\hline & Sample & Contact angle $\left(^{\circ}\right)$ \\
\hline 1 & $\mathrm{Co}-(\mathrm{BimH})_{3}$ & 21 \\
2 & $\mathrm{Co}-\left(\mathrm{BimH} / \mathrm{Me}_{2}\right)_{3}$ & 51 \\
3 & $\mathrm{Co}-\left(\mathrm{BimH} / \mathrm{Cl}_{2}\right)_{3}$ & 49 \\
4 & $\mathrm{Co}-\left(\mathrm{BimH} / \mathrm{NO}_{2}\right)_{3}$ & 46 \\
5 & $\mathrm{Co}-\left(\mathrm{BimH} / \mathrm{F}_{4}\right)_{3}$ & 69 \\
6 & $\mathrm{Co}-\left(\mathrm{BimH} / \mathrm{SO}_{3} \mathrm{~K}\right)_{3}$ & 16 \\
7 & $\mathrm{Co}-\left(\mathrm{BimC}_{4} \mathrm{H}\right)_{3}$ & 57 \\
8 & $\mathrm{Co}-\left(\mathrm{BimC}_{6}+2\right)_{3}$ & 62 \\
9 & $\mathrm{Co}-\left(\mathrm{BimC}_{10} \mathrm{H}\right)_{3}$ & 64 \\
10 & $\mathrm{Co}-\left(\mathrm{BimC}_{3} \mathrm{~F}_{8}\right)_{3}$ & 109 \\
11 & $\mathrm{Co}-(\mathrm{BimH})_{2}\left(\mathrm{BimC}_{3} \mathrm{PA}\right)$ & 16 \\
12 & $\mathrm{Co}-\left(\mathrm{BimC} \mathrm{F}_{8}\right)_{2}\left(\mathrm{BimC}_{3} \mathrm{PA}\right)$ & 52 \\
13 & $\mathrm{Co}-\left(\mathrm{BimC}_{4} \mathrm{CO}_{2} \mathrm{~K}\right)_{3}$ & 23 \\
\hline
\end{tabular}




\section{Electrochemical Data}

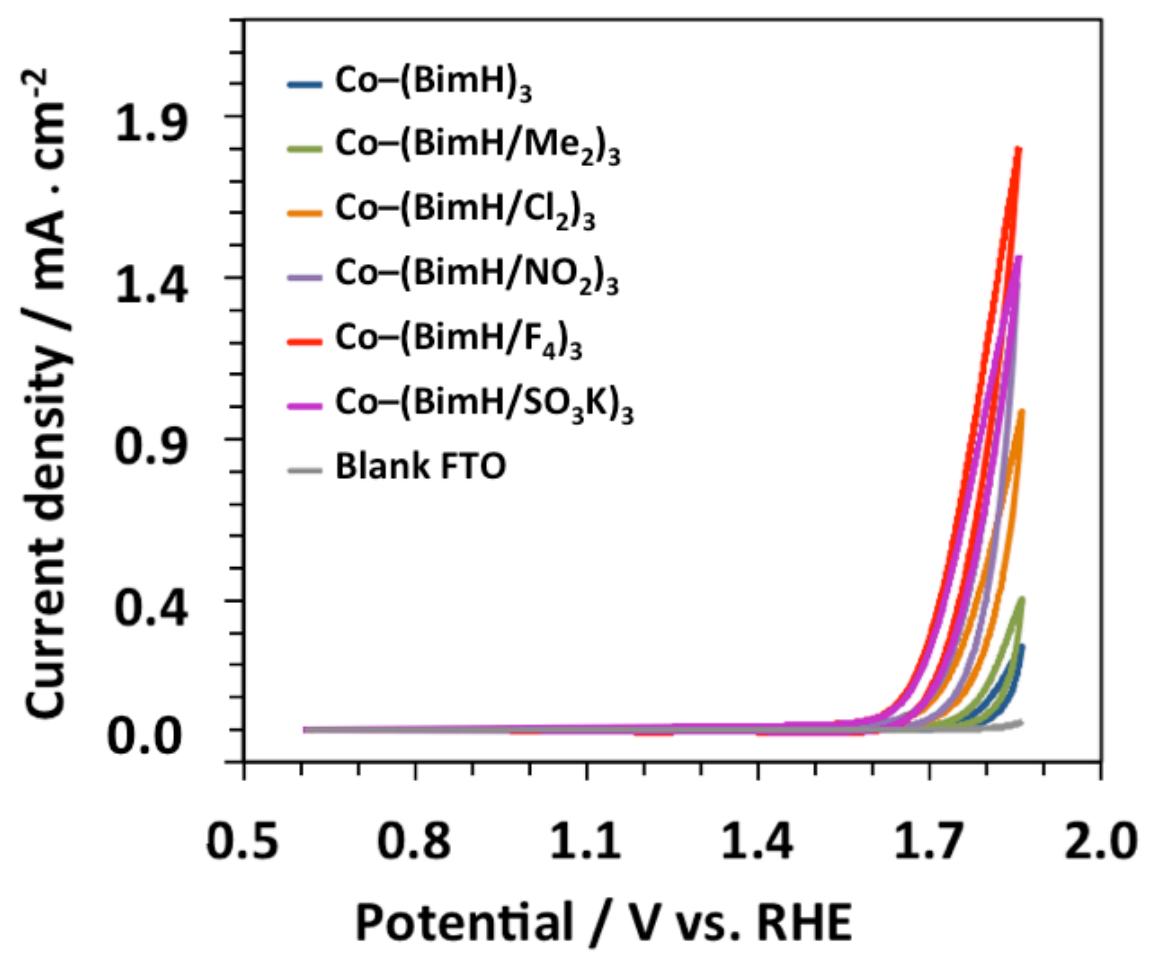

Figure S1. Cyclic voltammogram of trisbenzimidazole Co-complexes with benzene-ring-substituted variants $(\mathbf{B i m H} / \mathbf{R})_{3}$, in $0.1 \mathrm{M}$ potassium phosphate buffer, $\mathrm{pH} 7$ and $50 \mathrm{mV} \cdot \mathrm{s}^{-1}$ scan rate. Loading: $5 \times 10^{-10} \mathrm{~mol} \cdot \mathrm{cm}^{-2}$.

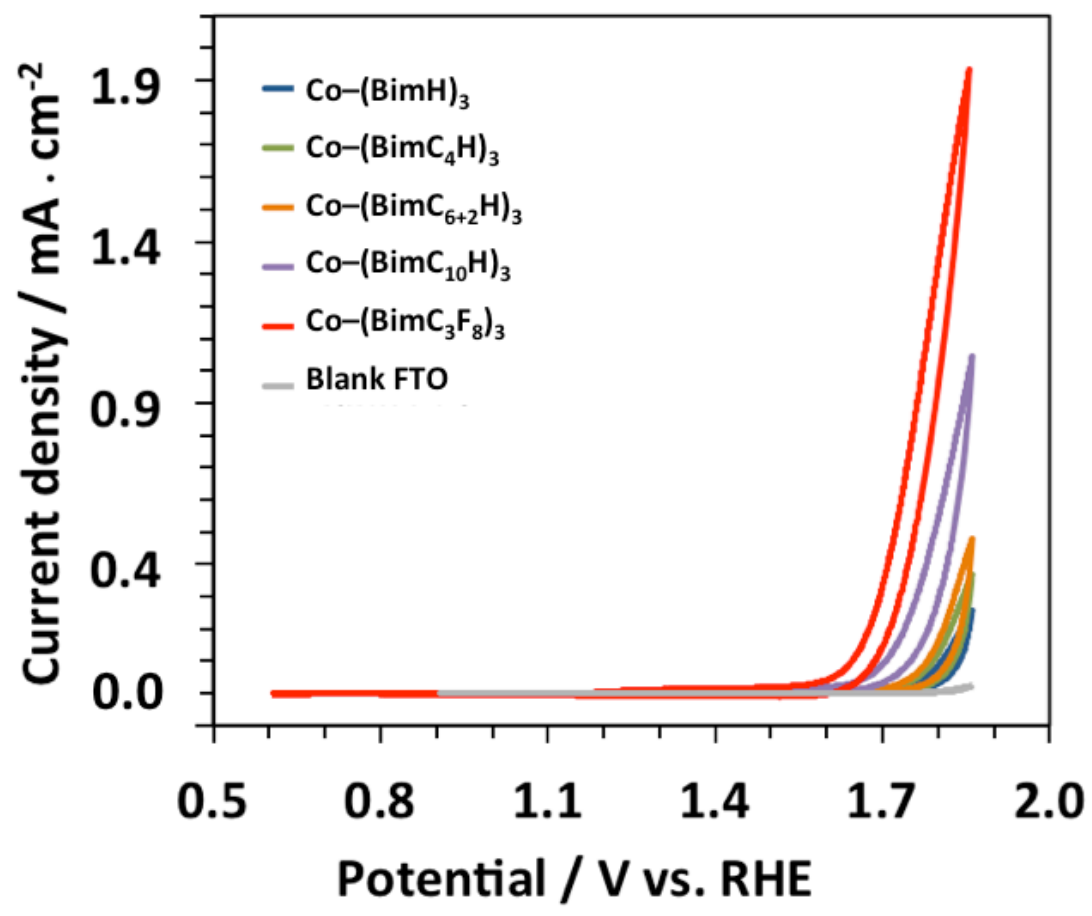

Figure S2. Cyclic voltammogram of Co-complexes with $\mathrm{N}$-alkylated trisbenzimidazole derivatives $(\mathbf{B i m R})_{3}$, in $0.1 \mathrm{M}$ potassium phosphate buffer, $\mathrm{pH} 7$ and $50 \mathrm{mV} \cdot \mathrm{s}^{-1}$ scan rate. Loading: $5 \times 10^{-10} \mathrm{~mol} \cdot \mathrm{cm}^{-2}$. 


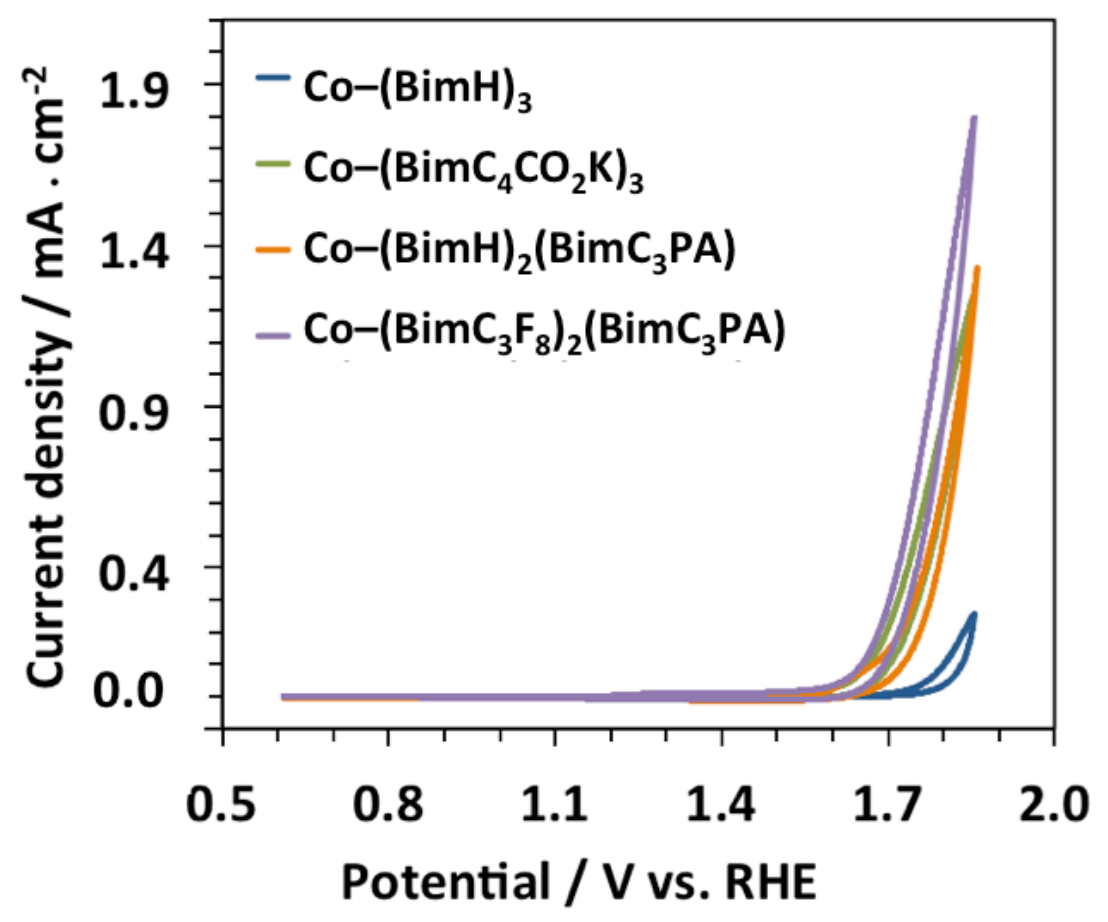

Figure S3. Cyclic voltammogram of Co-complexes with $\mathrm{N}$-alkylated trisbenzimidazole derivatives $(\mathbf{B i m R})_{3}$ with carboxylic and phosphonic acids, in $0.1 \mathrm{M}$ potassium phosphate buffer, $\mathrm{pH} 7$ and $50 \mathrm{mV} \cdot \mathrm{s}^{-1} \mathrm{scan}$ rate. Loading: $5 \times 10^{-10} \mathrm{~mol} \cdot \mathrm{cm}^{-2}$.

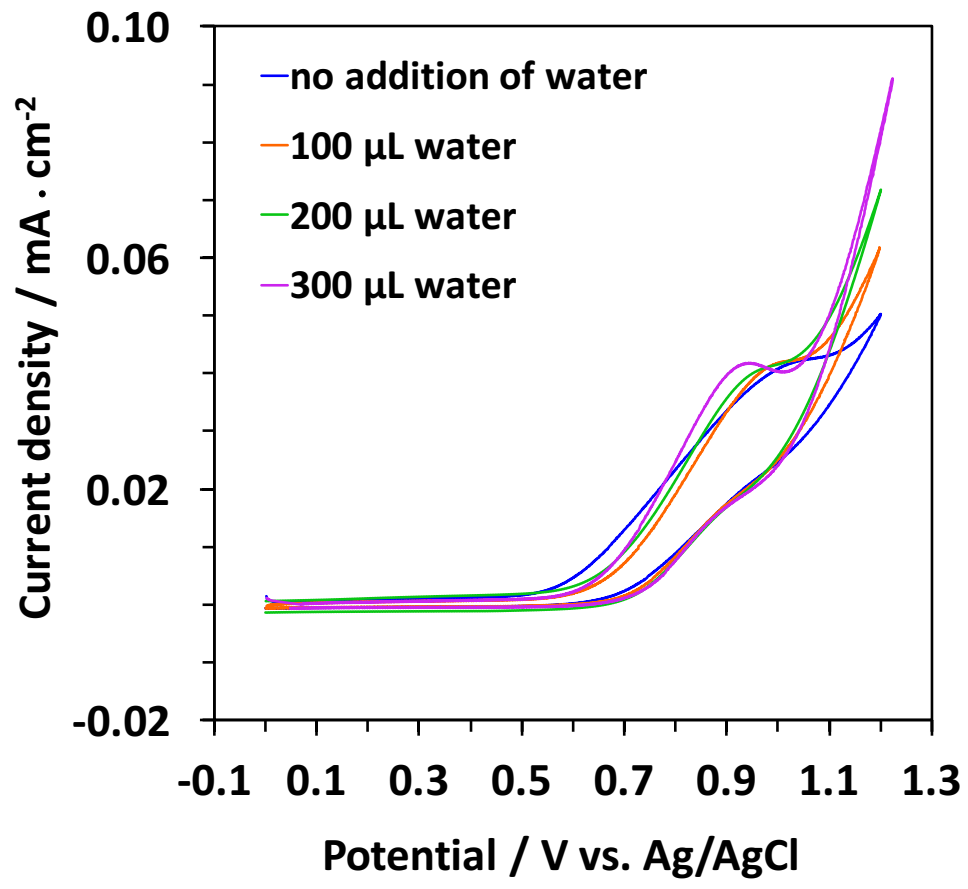

Figure S4. Cyclic voltammograms for $0.5 \mathrm{mM}$ Co- $\left(\mathbf{B i m C}_{3} \mathbf{F}_{\mathbf{8}}\right)_{3}$ in DMF $(10 \mathrm{~mL})$. No water added (blue curve) and $100-300 \mu \mathrm{L}$ of

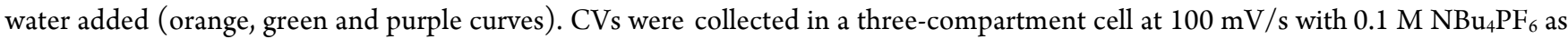
supporting electrolyte, $3 \mathrm{~mm}$ diameter glassy carbon working electrode, Pt counterelectrode, and $\mathrm{Ag} / \mathrm{AgCl}$ reference electrode. The midpoint potential for the redox event before the onset of the catalytic wave is $0.86 \mathrm{~V}$ vs $\mathrm{Ag} / \mathrm{AgCl}$. 


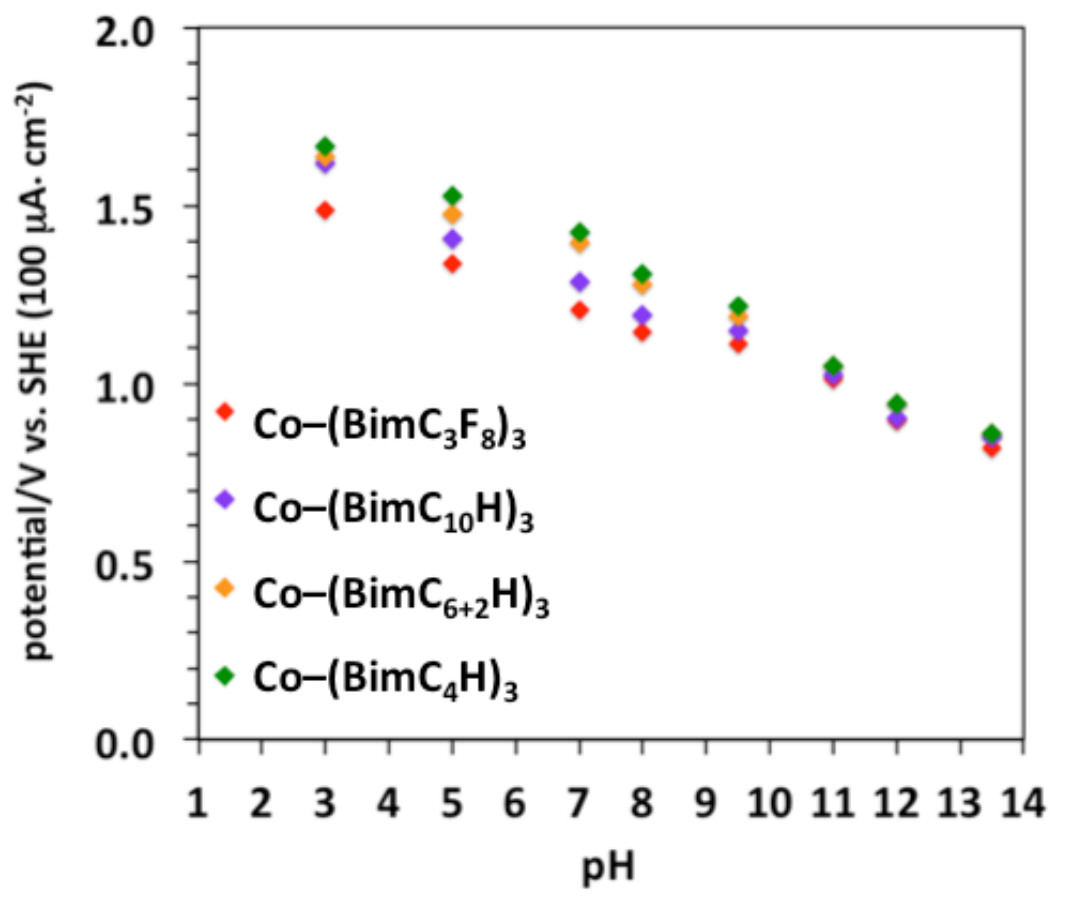

Figure S5. Dependence of the WOR onset potential (measured at $100 \mu \mathrm{A} \cdot \mathrm{cm}^{-2}$ ) on the pH for Co complexes with $\mathrm{N}$-alkylated trisbenzimidazole derivatives $(\operatorname{BimR})_{3}$ (electrolyte concentration $0.1 \mathrm{M}$, in the presence of phosphate; $\Gamma=5.0 \times 10^{-10} \mathrm{~mol} \cdot \mathrm{cm}^{-2} ; \mathrm{scan}$ rate $50 \mathrm{mV} / \mathrm{s})$. Slope is $-(60-75) \mathrm{mV}$ per $\mathrm{pH}$ unit. Electrolyte concentration: $0.1 \mathrm{M}$. Scan rate $50 \mathrm{mV} / \mathrm{s}$.

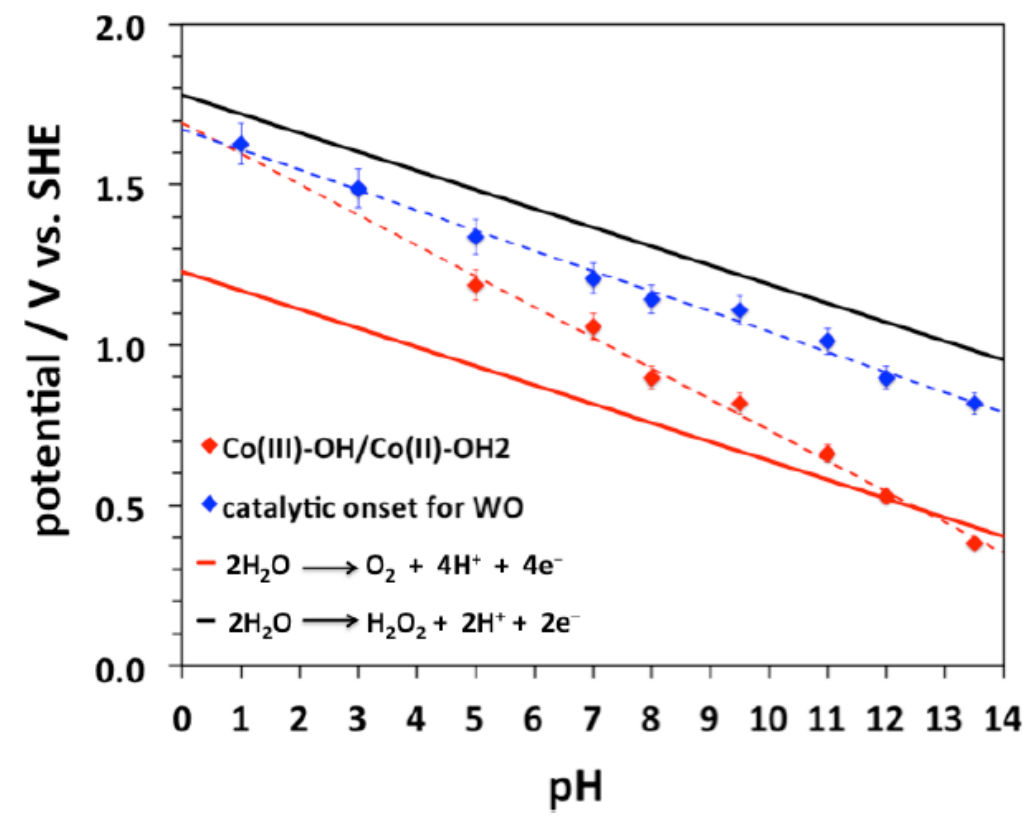

Figure S6. Blue diamonds $\diamond:$ Dependence of the WOR onset potential (measured at $\left.100 \mu \mathrm{A} \cdot \mathrm{cm}^{-2}\right)$ on the $\mathrm{pH}$ for $\mathbf{C o}-\left(\mathbf{B i m} \mathbf{C}_{3} \mathbf{F}_{\mathbf{8}}\right)_{3}$ (electrolyte concentration $0.1 \mathrm{M}$, in the presence of phosphate; $\Gamma=5.0 \times 10^{-10} \mathrm{~mol} \cdot \mathrm{cm}^{-2}$; scan rate $50 \mathrm{mV} / \mathrm{s}$ ). Slope is $-63 \mathrm{mV}$ per $\mathrm{pH}$ unit. Red diamonds $\diamond$ : Dependence of the redox couple midpoint potential on the $\mathrm{pH}$, slope $-95 \mathrm{mV}$ per $\mathrm{pH}$ unit. Red line: thermodynamic potential for water oxidation to oxygen via $4 \mathrm{H}^{+} / 4 \mathrm{e}^{-}$transfer. Black line: thermodynamic potential for water oxidation to $\mathrm{H}_{2} \mathrm{O}_{2}$ via $2 \mathrm{H}^{+} / 2 \mathrm{e}^{-}$transfer. 
A
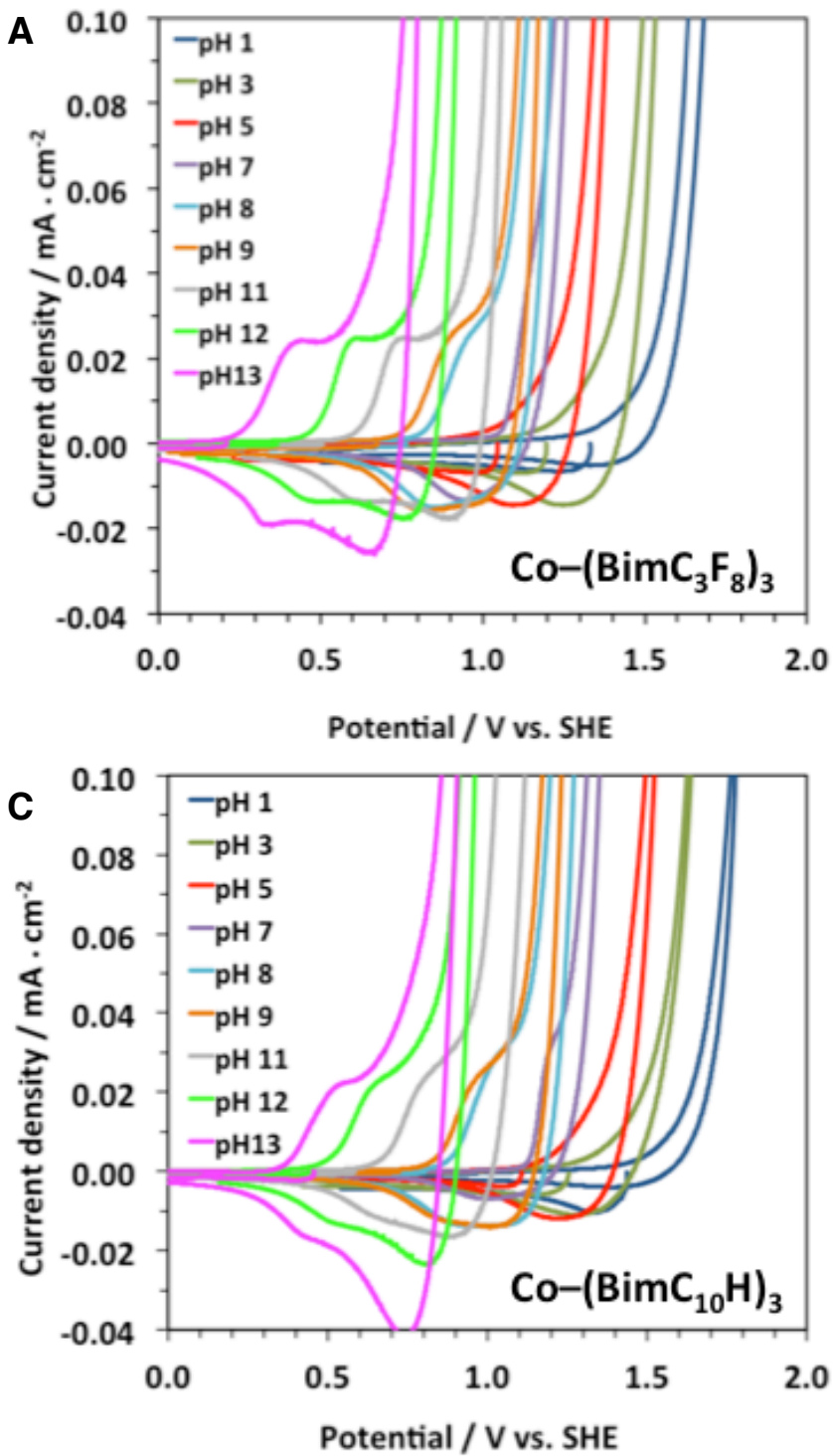
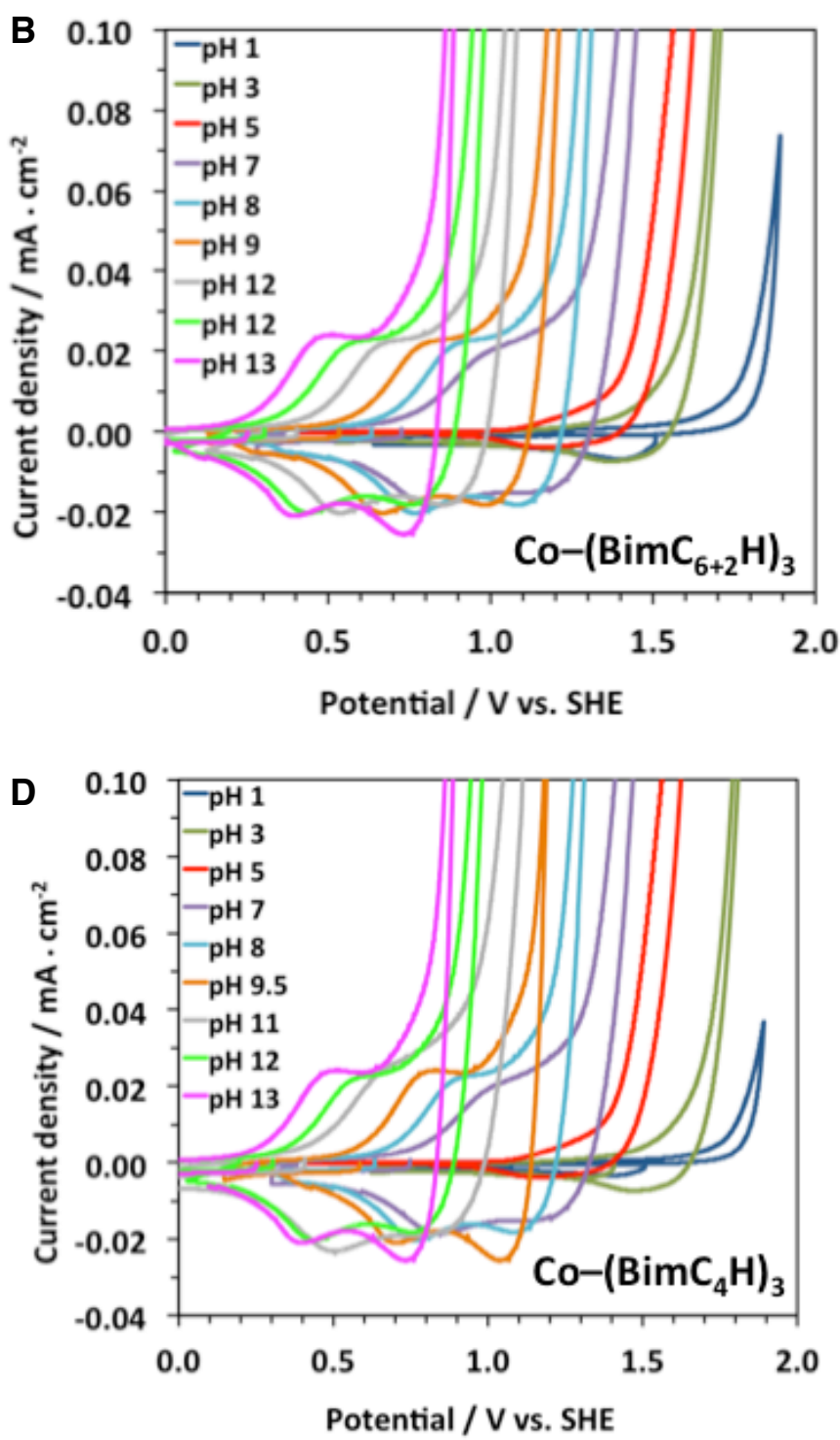

Figure S7. Magnified view of the CVs collected for Co complexes with $\mathrm{N}$-alkylated trisbenzimidazole derivatives $(\mathbf{B i m R})_{3}(\Gamma=5.0 \times$ $\left.10^{-10} \mathrm{~mol} \cdot \mathrm{cm}^{-2}\right)$ at different $\mathrm{pH}$ in the range $1-13(0.1 \mathrm{M}$ electrolyte concentration, scan rate $50 \mathrm{mV} / \mathrm{s})$. 


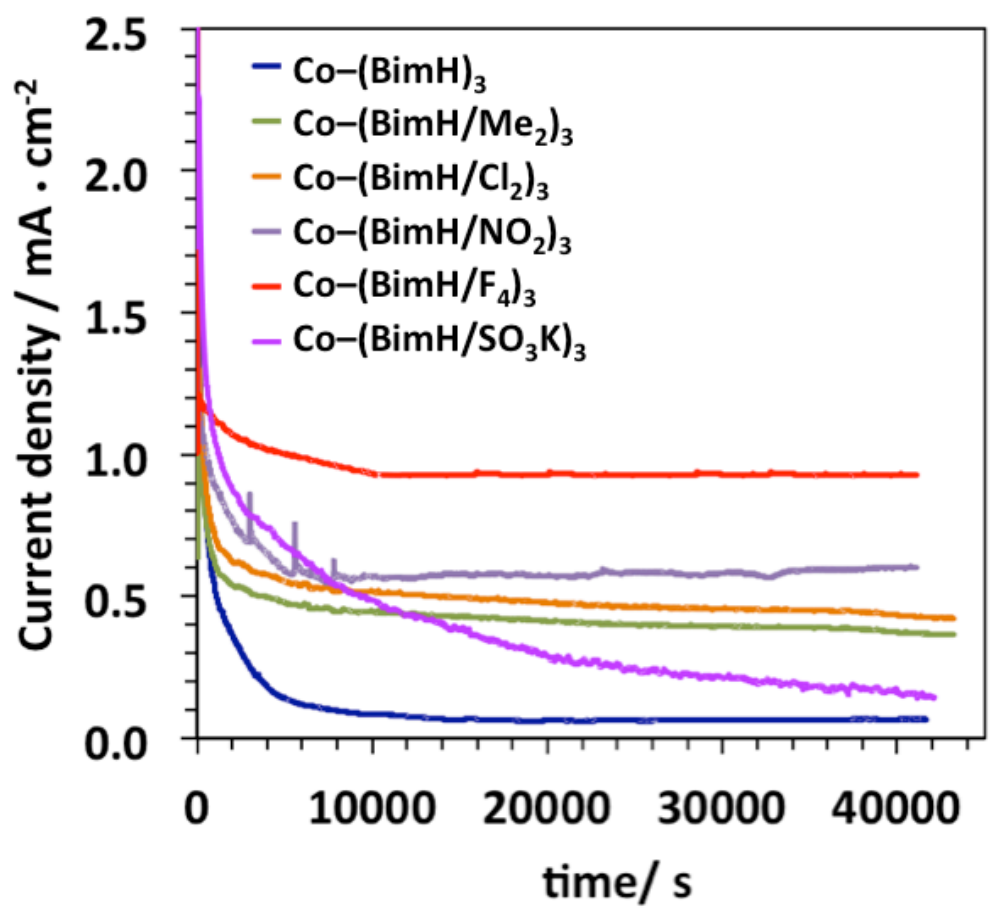

Figure S8. Controlled potential electrolysis of trisbenzimidazole Co-complexes with benzene-ring-substituted variants $(\mathbf{B i m} \mathbf{H} / \mathbf{R})_{3}$, at $1.91 \mathrm{~V}$ vs. RHE, in $0.1 \mathrm{M}$ potassium phosphate buffer, $\mathrm{pH}$. Loading: $2 \times 10^{-9} \mathrm{~mol} \cdot \mathrm{cm}^{-2}$.

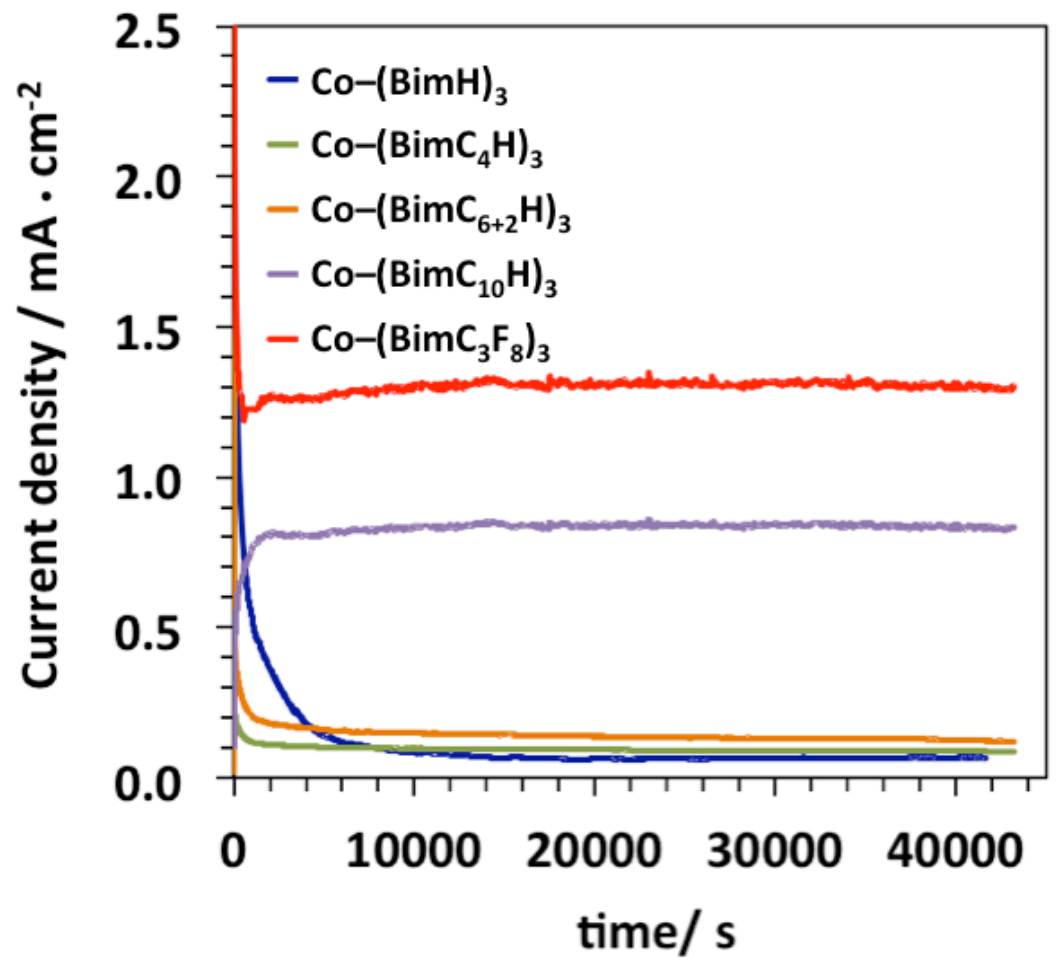

Figure S9. Controlled potential electrolysis of Co-complexes with $\mathrm{N}$-alkylated trisbenzimidazole derivatives $(\mathbf{B i m R})_{3}$, at $1.91 \mathrm{~V}$ vs. RHE, in $0.1 \mathrm{M}$ potassium phosphate buffer, $\mathrm{pH}$. Loading: $2 \times 10^{-9} \mathrm{~mol} \cdot \mathrm{cm}^{-2}$. 


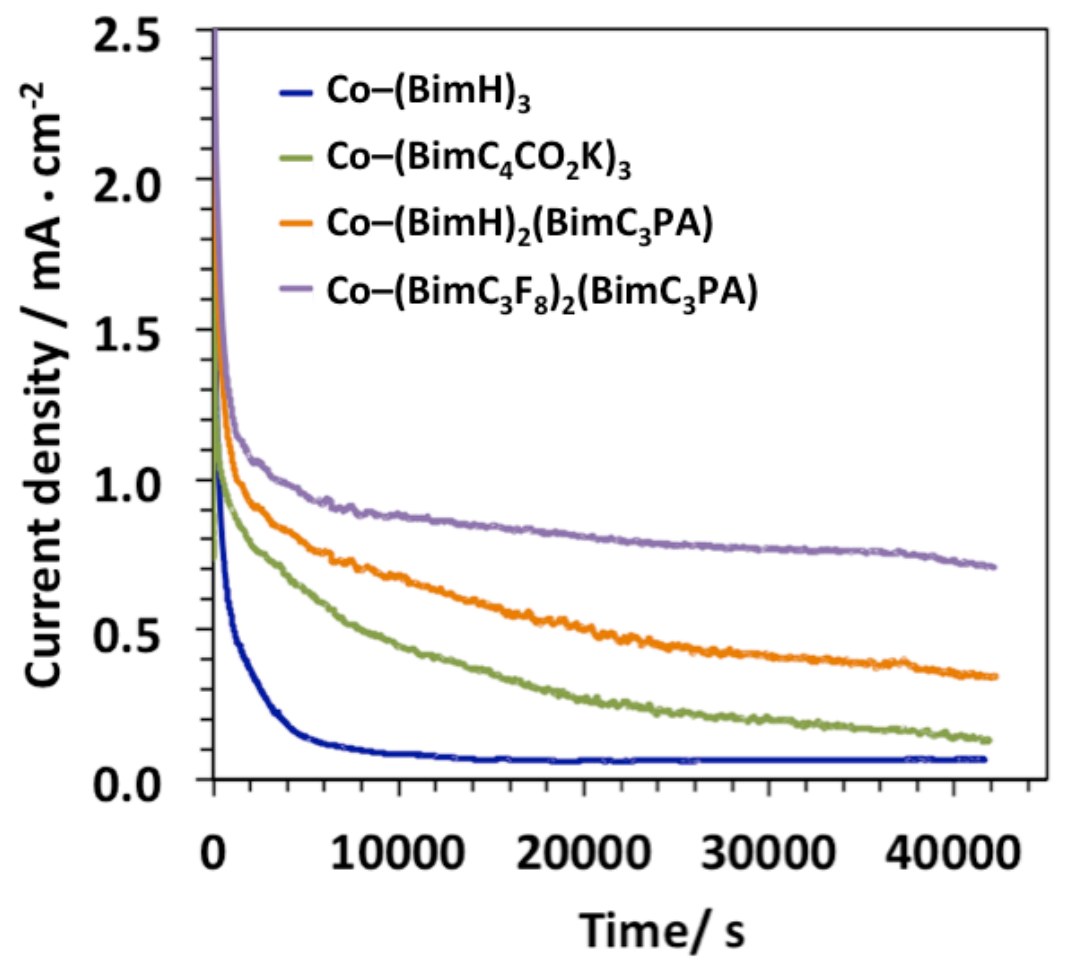

Figure S10. Controlled potential electrolysis of Co-complexes with $\mathrm{N}$-alkylated trisbenzimidazole derivatives (BimR) ${ }_{3}$ with carboxylic and phosphonic acids, at $1.91 \mathrm{~V}$ vs. RHE, in $0.1 \mathrm{M}$ potassium phosphate buffer, $\mathrm{pH} 7$. Loading: $2 \times 10^{-9} \mathrm{~mol} \cdot \mathrm{cm}^{-2}$.

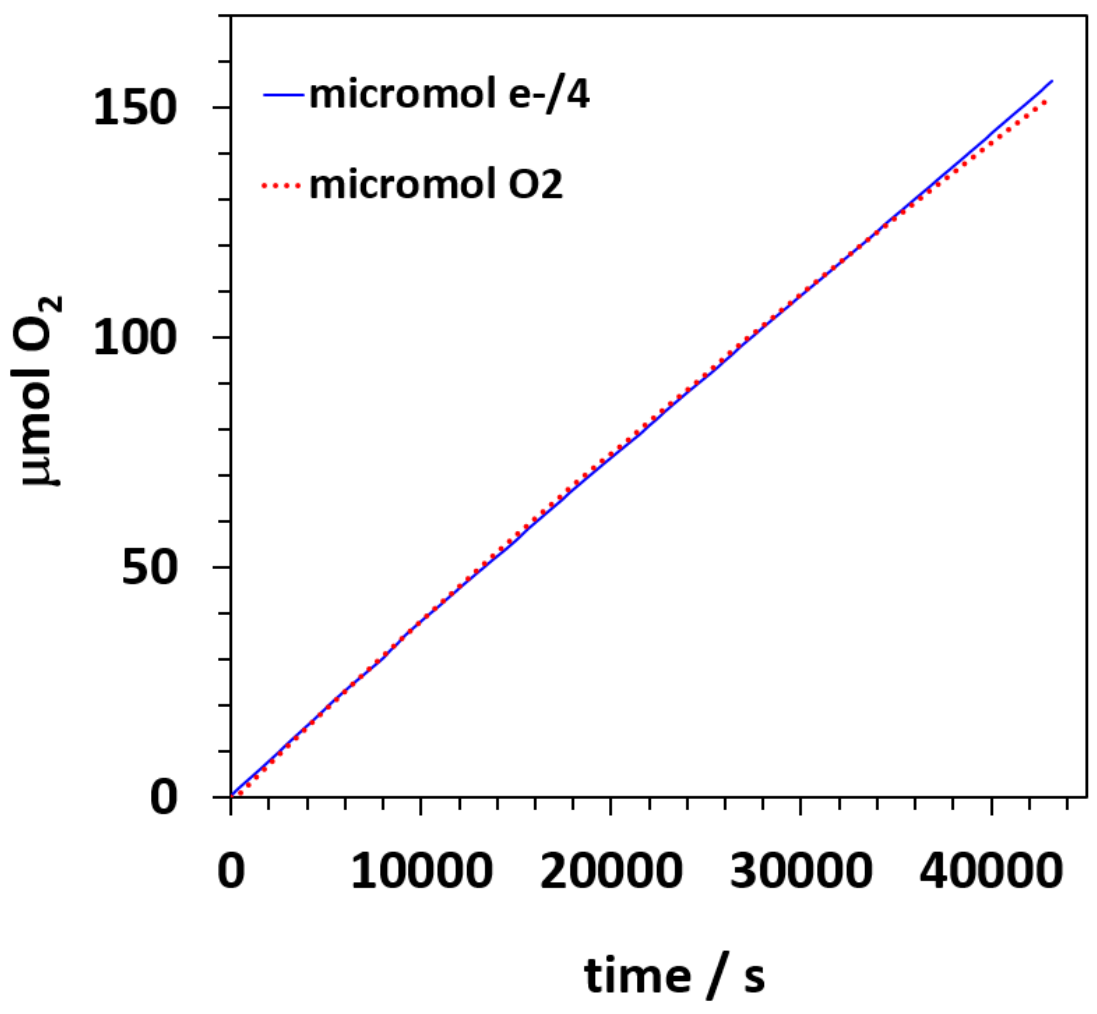

Figure S11. Oxygen evolution during controlled potential electrolysis Co- $\left(\mathbf{B i m C}_{3} \mathbf{F}_{8}\right)_{3}$ at $1.91 \mathrm{~V}$ vs. RHE, in $0.1 \mathrm{M}$ potassium phosphate buffer, pH 7 . Loading: $2 \times 10^{-9} \mathrm{~mol} \cdot \mathrm{cm}^{-2}$. 


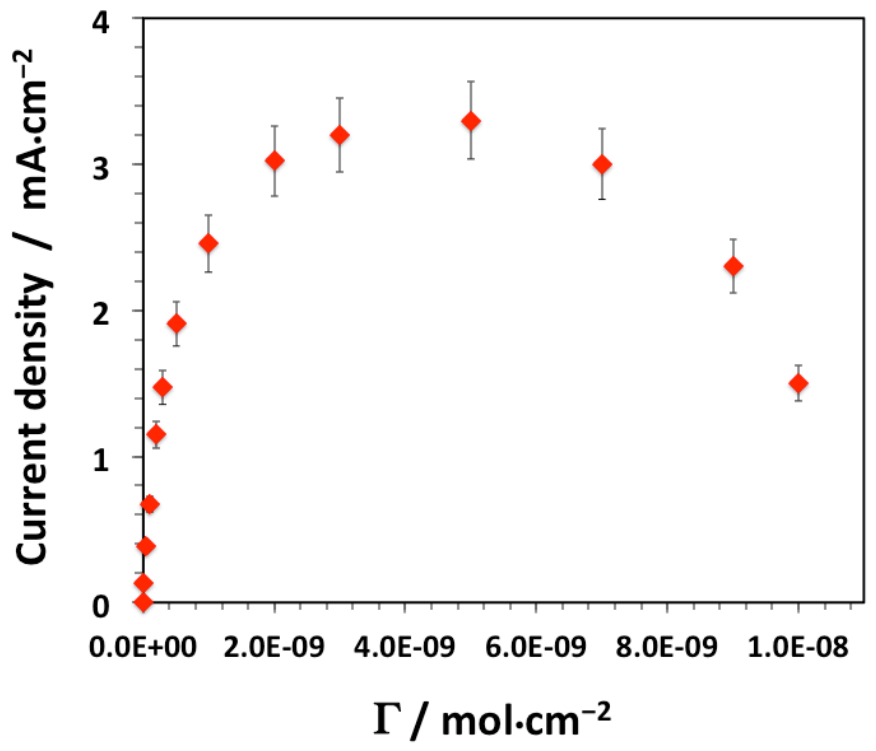

Figure S12. Dependence of peak current for the catalytic wave at $1.85 \mathrm{~V}$ vs. $\mathrm{RHE}$ with the catalyst loading, for $\mathrm{Co}-\left(\mathrm{BimC} \mathrm{F}_{8}\right)_{3}(0.1 \mathrm{M}$ potassium phosphate buffer, $\mathrm{pH} 7$, loadings $\Gamma=1.0 \times 10^{-11}-1.0 \times 10^{-8} \mathrm{~mol} \mathrm{~cm}{ }^{-2}$ ).

To investigate the ability of our catalytic system to perform in regimes with higher current densities, we have performed a chronopotentiometry experiment, with the current density fixed at $10 \mathrm{~mA} \mathrm{~cm}^{-2}$ (Figure S13). While it has been possible to achieve sustained water oxidation at this current density, the overpotential necessary for that is significantly higher than that necessary for our original range of current densities of $\sim 1-2 \mathrm{~mA} \mathrm{~cm}^{-2}$.

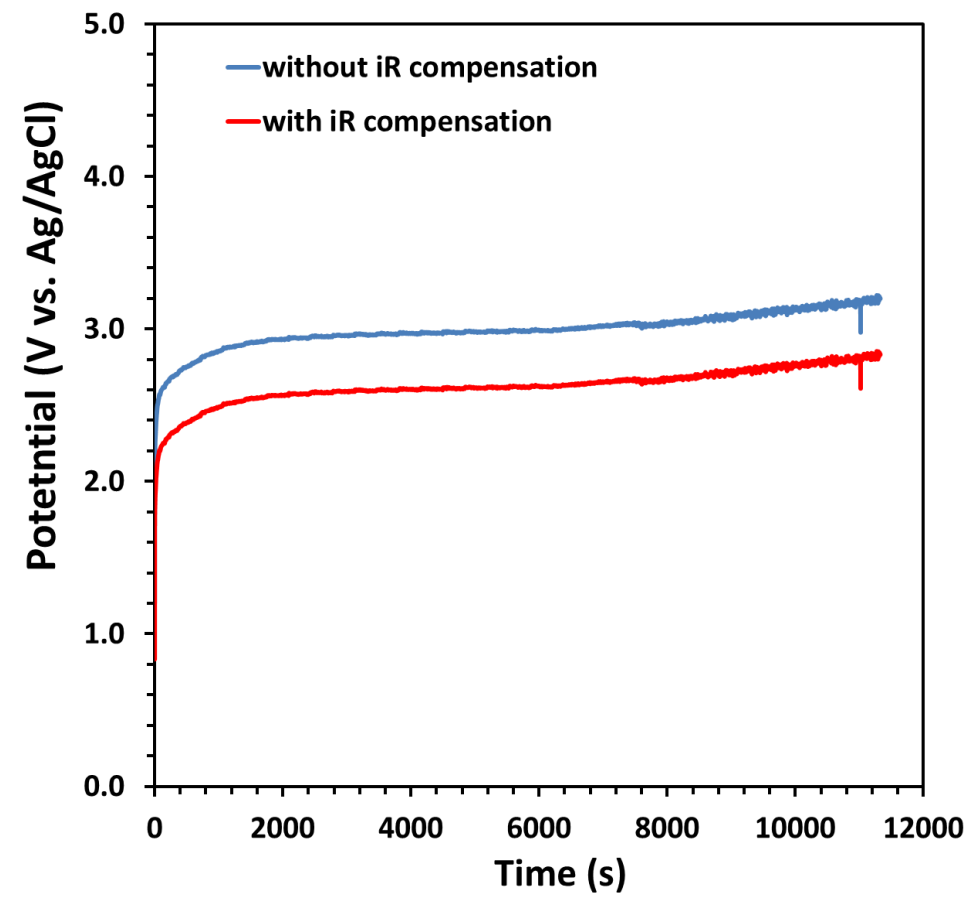

Figure S13. Chronopotentiometry of $\mathbf{C o -}\left(\mathbf{B i m C}_{3} \mathbf{F}_{\mathbf{8}}\right)_{3} @ \mathrm{FTO}$ electrode at $10 \mathrm{~mA} \mathrm{~cm} \mathrm{c}^{-2}$ in $0.1 \mathrm{M}$ potassium phosphate buffer, pH 7. Loading: $5 \times 10^{-9} \mathrm{~mol} \mathrm{~cm}^{-2}$ (chosen to maximize current density - refer to Figure S12). 
Table S4. Onset potential and TOF obtained with electrodes modified with $0.5-2 \times 10^{-9}$ mol. $\mathrm{cm}^{-2}$ loading of different Cotrisbenzimidazoles.

\begin{tabular}{|c|c|c|c|c|}
\hline & Sample & Onset potential (V vs. RHE) ${ }^{a}$ & Current density $\left(\mathrm{mA} \cdot \mathrm{cm}^{-2}\right)^{b}$ & TOF $\left(s^{-1}\right)^{c}$ \\
\hline 1 & $\mathrm{Co}-(\mathrm{BimH})_{3}$ & 1.86 & & - \\
\hline 2 & $\mathrm{Co}-\left(\mathrm{BimH} / \mathrm{Me}_{2}\right)_{3}$ & 1.74 & 0.37 & 0.48 \\
\hline 4 & $\mathrm{Co}-\left(\mathrm{BimH} / \mathrm{NO}_{2}\right)_{3}$ & 1.63 & 0.63 & 0.82 \\
\hline 5 & $\mathrm{Co}-\left(\mathrm{BimH} / \mathrm{F}_{4}\right)_{3}$ & 1.62 & 0.94 & 1.22 \\
\hline 7 & $\mathrm{Co}-\left(\mathrm{BimC}_{4} \mathrm{H}\right)_{3}$ & 1.83 & 0.09 & 0.12 \\
\hline 8 & $\mathrm{Co}-\left(\mathrm{BimC}_{6+2} \mathrm{H}\right)_{3}$ & 1.81 & 0.13 & 0.16 \\
\hline 9 & $\mathrm{Co}-\left(\mathrm{BimC}_{10} \mathrm{H}\right)_{3}$ & 1.69 & 0.86 & 1.11 \\
\hline 10 & $\mathrm{Co}-\left(\mathrm{BimC}_{3} \mathrm{~F}_{8}\right)_{3}$ & 1.61 & 1.42 & $1.83^{\mathrm{d}}$ \\
\hline
\end{tabular}

${ }^{\mathrm{a}}$ Onset potential: potential when current density reaches $100 \mu \mathrm{A} / \mathrm{cm}^{2}$ during $\mathrm{CV}$ at $1 \mathrm{mV} / \mathrm{s}$

${ }^{\mathrm{b}}$ Sustained current density reached during the CPE experiment at 1.91 V vs. RHE (see Figure S8 and Figure S9)

${ }^{\mathrm{c}}$ TOF: calculated from the steady current reached during the cpe experiment at $1.91 \mathrm{~V}$ vs. RHE

d TOF: calculated from the oxygen measurement during the cpe experiment at $1.91 \mathrm{~V}$ vs. RHE (see Figure S11).

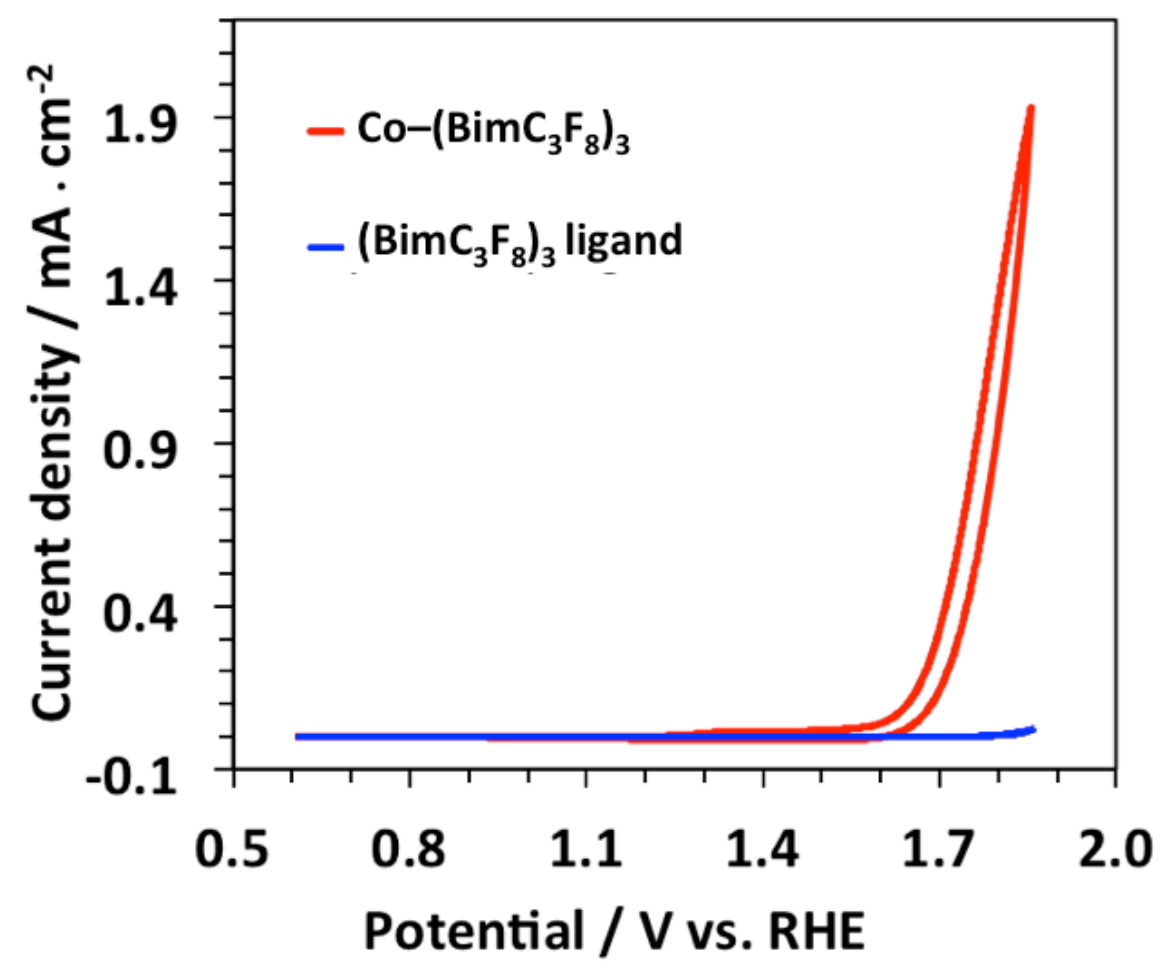

Figure S14. Cyclic voltammograms of $\mathbf{C o -}\left(\mathbf{B i m C}_{3} \mathbf{F}_{\mathbf{8}}\right)_{3}$ and $\left(\mathbf{B i m C}_{3} \mathbf{F}_{\mathbf{8}}\right)_{3}$ ligand $\left(\Gamma=5 \times 10^{-10} \mathrm{~mol} \cdot \mathrm{cm}^{-2}\right)$ at $\mathrm{pH} 7(0.1 \mathrm{M}$ potassium phosphate buffer and $50 \mathrm{mV} \cdot \mathrm{s}^{-1}$ scan rate). 


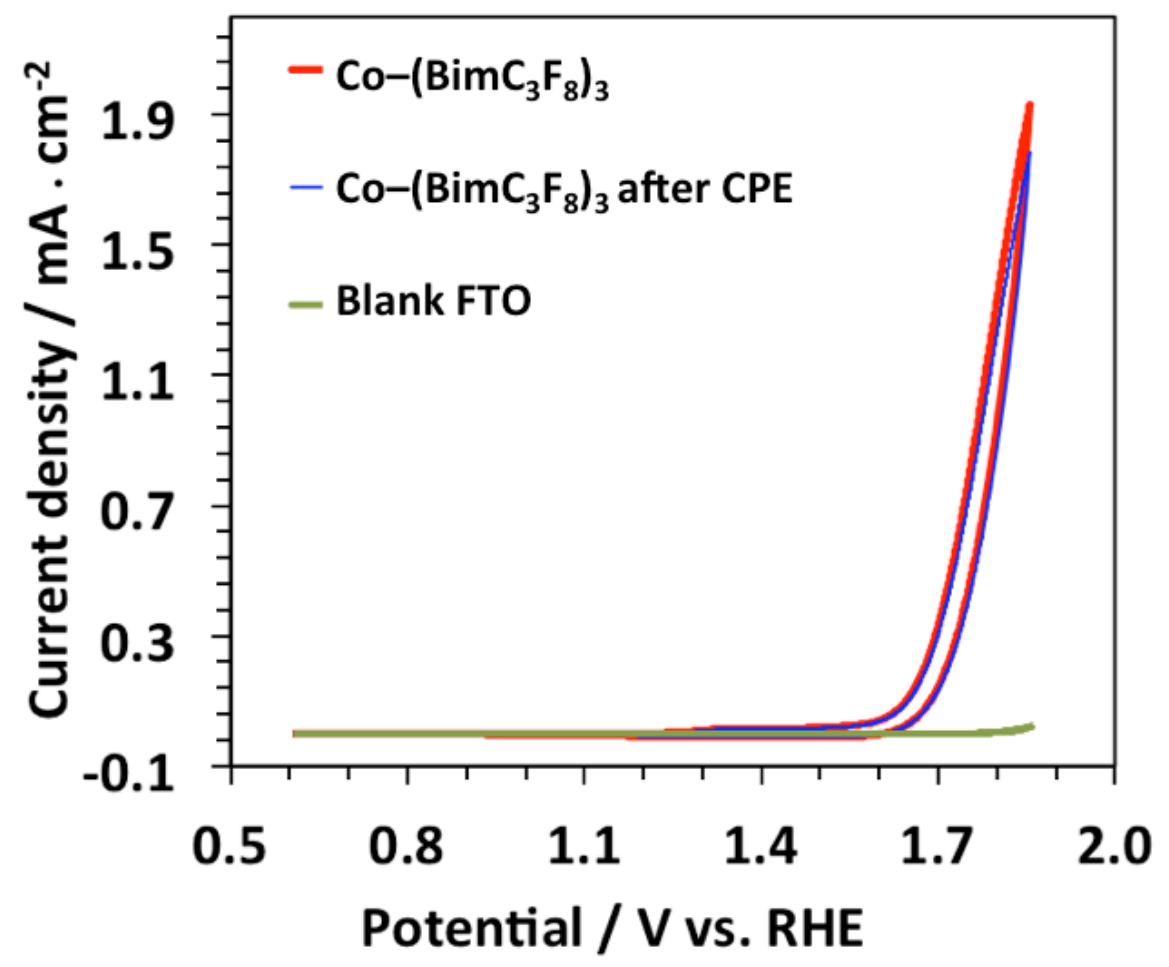

Figure S15. Cyclic voltammograms of Co- $\left(\mathbf{B i m C}_{3} \mathbf{F}_{8}\right)_{3}\left(\Gamma=5.0 \times 10^{-10} \mathrm{~mol} \cdot \mathrm{cm}^{-2}\right)$ before and after CPE for $12 \mathrm{~h}$ at $1.85 \mathrm{~V}$ vs. RHE at pH 7 (0.1 M potassium phosphate buffer).

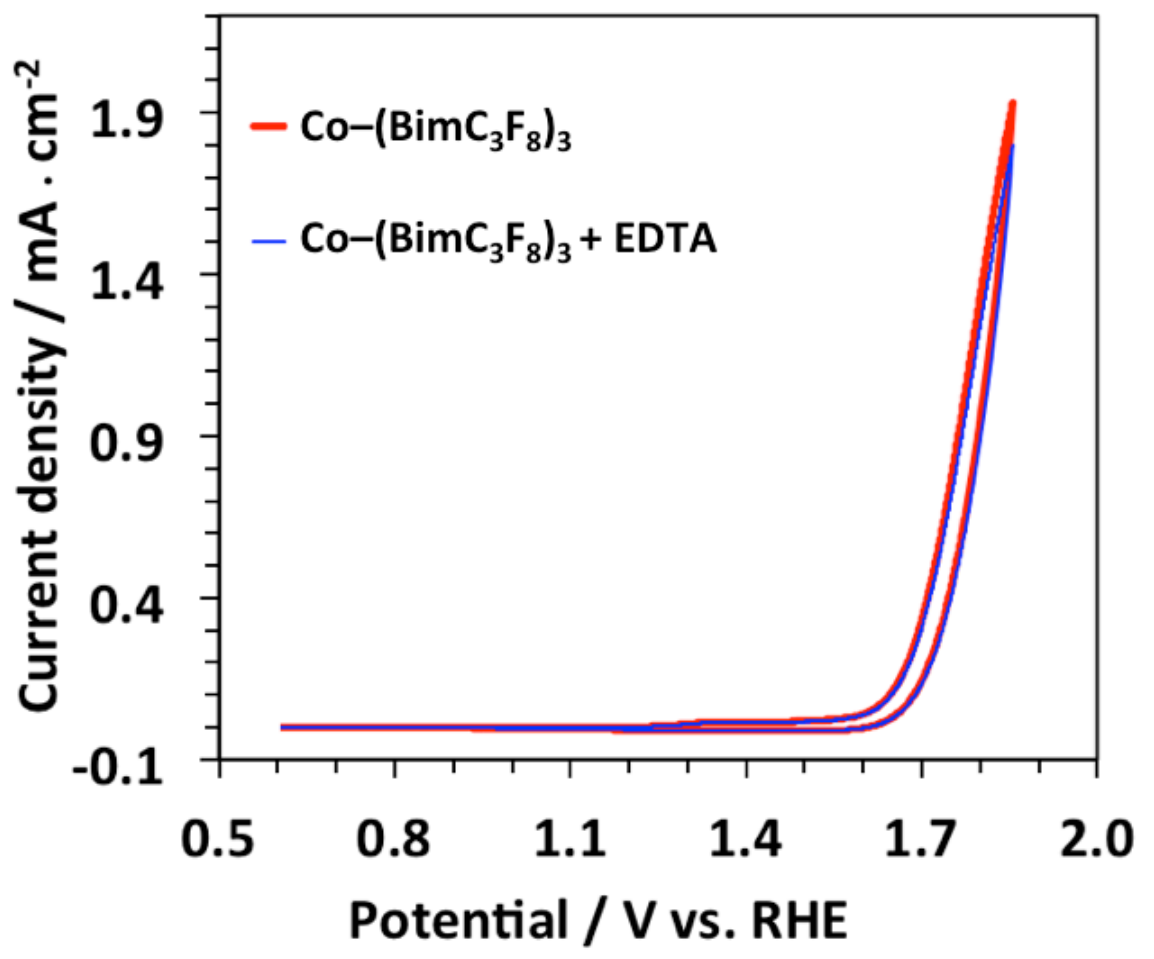

Figure S16. Cyclic voltammograms of $\mathbf{C o -}\left(\mathbf{B i m C}_{3} \mathbf{F}_{8}\right)_{3}\left(\Gamma=5.0 \times 10^{-10} \mathrm{~mol} \cdot \mathrm{cm}^{-2}\right)$ after addition of EDTA $0.05 \mathrm{mM}(0.1 \mathrm{M}$ potassium phosphate buffer $\mathrm{pH} 7$ and scan rate $50 \mathrm{mV} / \mathrm{s})$. 


\section{Spectroscopic Characterization of the Co-complexes after}

\section{electrochemistry}

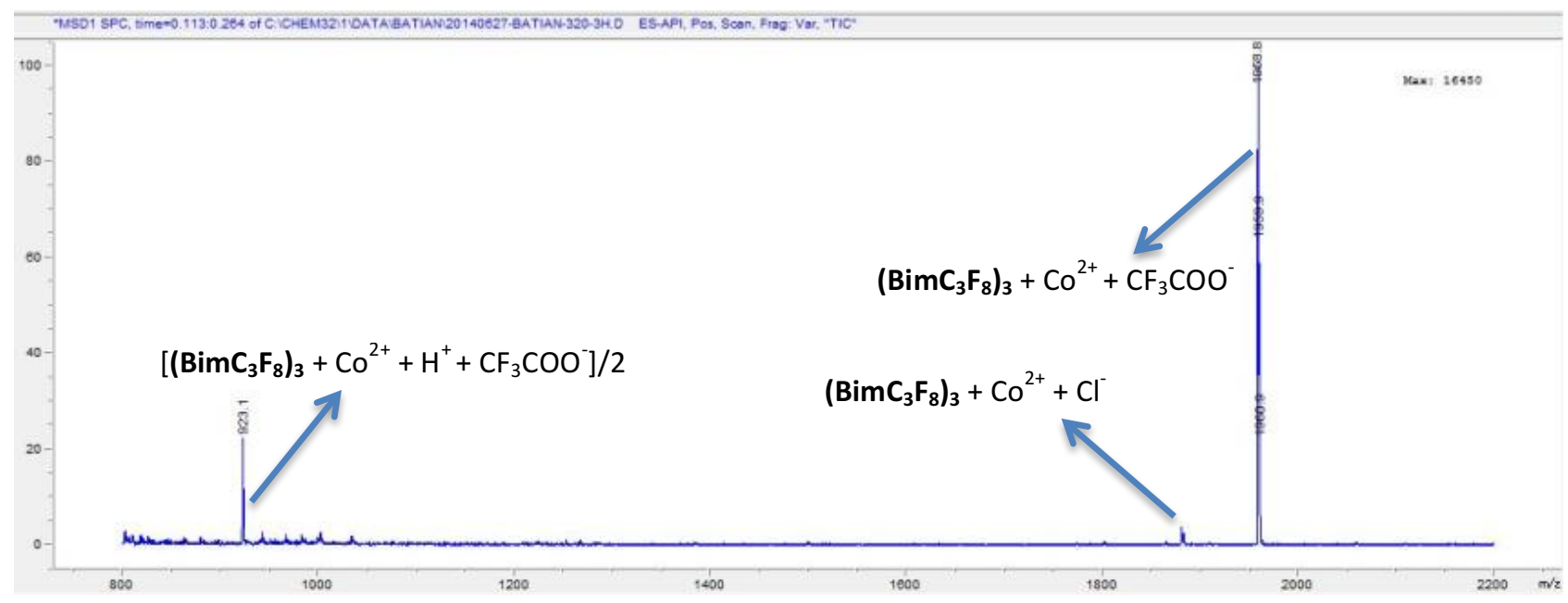

Figure S17. Mass spectra of $\mathbf{C o -}\left(\mathbf{B i m C}_{3} \mathbf{F}_{8}\right)_{3}$ after controlled potential electrolysis at $1.91 \mathrm{~V}$ vs. RHE for $12 \mathrm{~h}(0.1 \mathrm{M}$ potassium phosphate buffer, $\mathrm{pH} 7$ ).
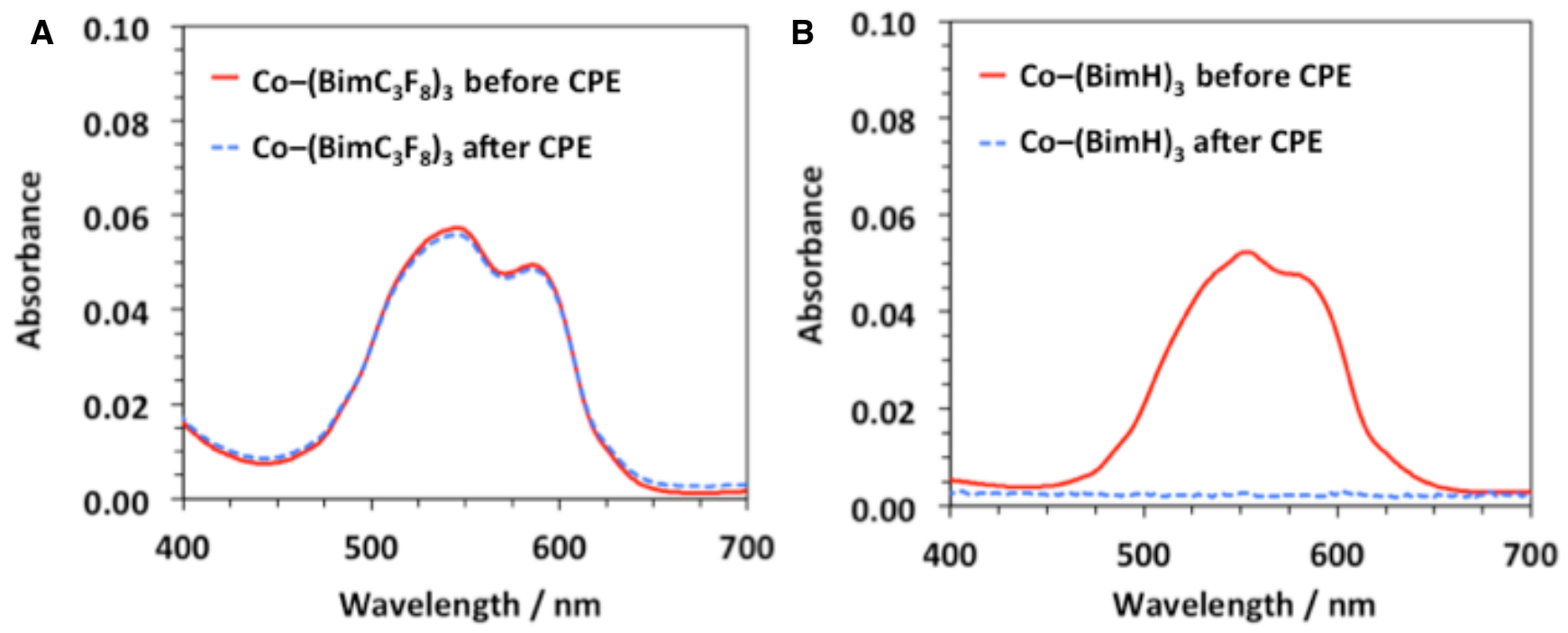

Figure S18. UV-Vis absorption spectra of $\mathbf{C o}-\left(\mathbf{B i m C}_{3} \mathbf{F}_{8}\right)_{3}(\mathrm{~A})$ and $\mathbf{C o}-(\mathbf{B i m H})_{3}(\mathrm{~B})$ in methanol $\left(2 \times 10^{-5} \mathrm{M}\right)$, before and after controlled potential electrolysis at $1.91 \mathrm{~V}$ vs. RHE for $12 \mathrm{~h}$ ( $0.1 \mathrm{M}$ potassium phosphate buffer, $\mathrm{pH} 7)$. 


\section{X-Ray Structure Determination}

\section{$\left[\mathrm{CoCl}\left(\mathrm{C}_{57} \mathrm{H}_{36} \mathrm{~F}_{51} \mathrm{~N}_{7}\right)\right] \mathrm{Cl} \cdot\left(\mathrm{C}_{4} \mathrm{H}_{8} \mathrm{O}\right)_{3} \cdot\left(\mathrm{H}_{2} \mathrm{O}\right)_{2}$}

Crystals grew as a six-sided plates showing a strong dichroism, appearing pink or deep purple depending on orientation. X-ray intensity data from were collected at 200(2) K using a Bruker D8 QUEST diffractometer equipped with a PHOTON 100 CMOS area detector and an Incoatec microfocus source (Mo K $\alpha$ radiation, $\lambda=0.71073 \AA$ ). ${ }^{11}$ Flash-cooling to lower temperatures resulted in visible cracking of the crystal. Some minor fractures were observed even at $200 \mathrm{~K}$. The material loses crystallinity at room temperature in air or under oil over a period of $\sim 1 \mathrm{hr}$. Data were collected on a large plate of approximate dimensions $0.56 \times$ $0.44 \times 0.24 \mathrm{~mm}^{3}$. Raw area detector data frames were reduced and corrected for absorption effects using the SAINT+ and SADABS programs. ${ }^{11}$ Final unit cell parameters were determined by least-squares refinement of 9691 reflections taken from the data set. The structure was solved by direct methods with SHELXT. ${ }^{12}$ Subsequent difference Fourier calculations and full-matrix leastsquares refinement against $F^{2}$ were performed with SHELXL-2014 ${ }^{12}$ using OLEX2. ${ }^{13}$

The compound crystallizes in the monoclinic system. The pattern of systematic absences in the intensity data was consistent with the space group $P 2_{1} / \mathcal{c}$, which was verified by structure solution. The asymmetric unit consists of one $\operatorname{CoCl}\left(\mathrm{C}_{57} \mathrm{H}_{36} \mathrm{~F}_{51} \mathrm{~N}_{7}\right)^{+}$cation, one chloride ion, three THF molecules of crystallization, and two water molecules. The crystal is afflicted with extensive disorder, affecting primarily the long fluorinated chains, but also interstitial solvent species. Two of the three independent $-\mathrm{C}_{8} \mathrm{~F}_{17}$ arms of the $\mathrm{C}_{57} \mathrm{H}_{36} \mathrm{~F}_{51} \mathrm{~N}_{7}$ ligand show pronounced positional disorder. The disorder was modeled with two components for chains C31-C38/F18-F34 and C50C57/F35-F51. No disorder was modeled for the third chain (C12-C19/F1-F17), but $d(\mathrm{C}-\mathrm{C})=1.55(2) \AA$ and $d(\mathrm{C}-\mathrm{F})=1.35 \AA$ distance restraints were applied, in addition to rigid-bond restraints (RIGU) for the $\mathrm{C}$ and $\mathrm{F}$ displacement parameters. The largest residual electron density peaks remaining after convergence are located near atoms of this arm, but trial modeling showed the minor disorder fraction to be small, $c a$. $<20 \%$, and difficulty was encountered in achieving a stable model. This is the reason the single 'average' positional model for this ligand arm was retained. The geometry of the other two $-\mathrm{C}_{8} \mathrm{~F}_{17}$ arms was then restrained to have similar 1,2- and 1,3- C-C and C-F distances using SHELX SAME instructions. Rigid-bond (RIGU) restraints were also applied to the anisotropic displacement parameters of atoms in these chains. One THF molecule (O3S) was restrained to adopt a similar geometry as the more well-ordered THF molecule O2S. The two water molecules are each disordered over two closely spaced positions, whose total occupancy was constrained to 1.0. In total 1116 restraints were used for the disorder modeling. All non-hydrogen atoms were refined with anisotropic displacement parameters except for the solvent species (isotropic). Hydrogen atoms bonded to carbon were in general located in difference maps before being included as riding atoms. Hydrogen atoms of the water molecules could not be located and were not calculated. The largest residual electron density peak in the final difference map is $1.03 \mathrm{e}^{-} / \AA^{3}$, located $1.30 \AA$ from $\mathrm{F}^{2}$. 


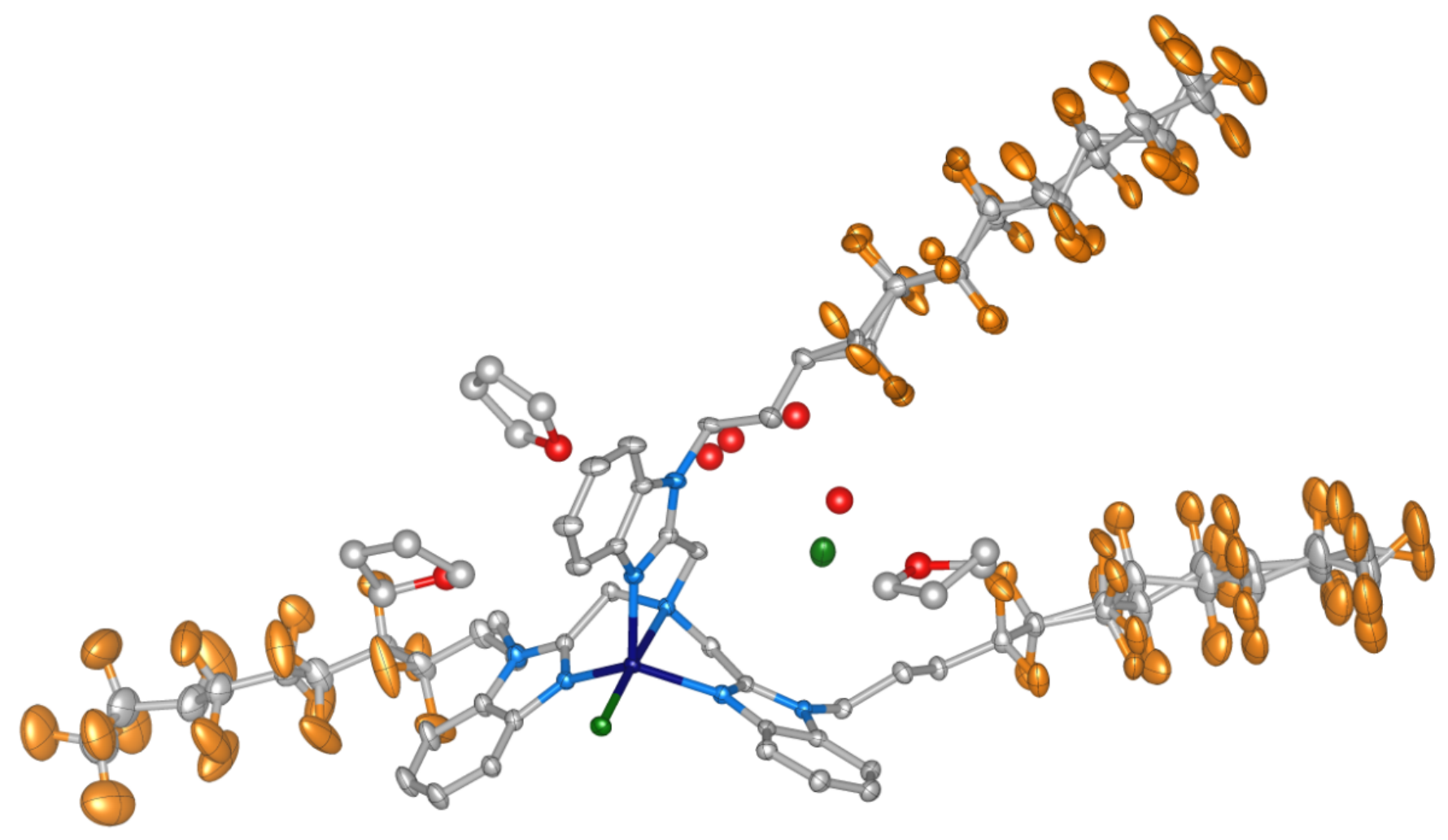

Figure S19. X-ray single crystal structure of $\mathbf{C o}\left(\mathbf{B i m C}_{3} \mathbf{F}_{8}\right)_{3}$. Hydrogen atoms are omitted for clarity. Cobalt (dark blue), nitrogen (blue), carbon (gray), chloride (green), oxygen (red) and fluorine (orange).

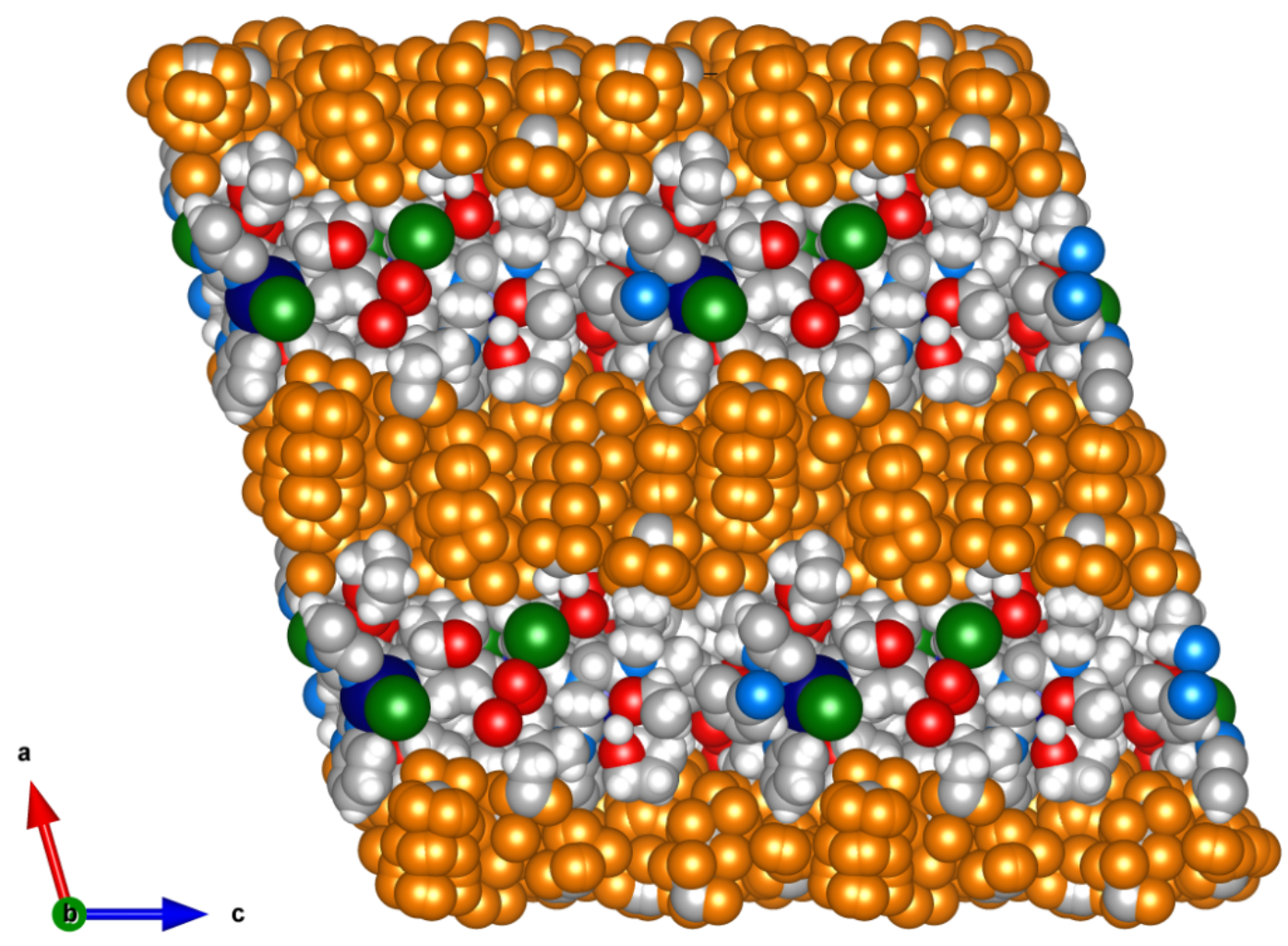

Figure S20. Packing viewed down the $b$ axis (Van Der Waals space-filling). Cobalt (dark blue), nitrogen (blue), carbon (gray), chloride (green), oxygen (red), hydrogen (white) and fluorine (orange). 


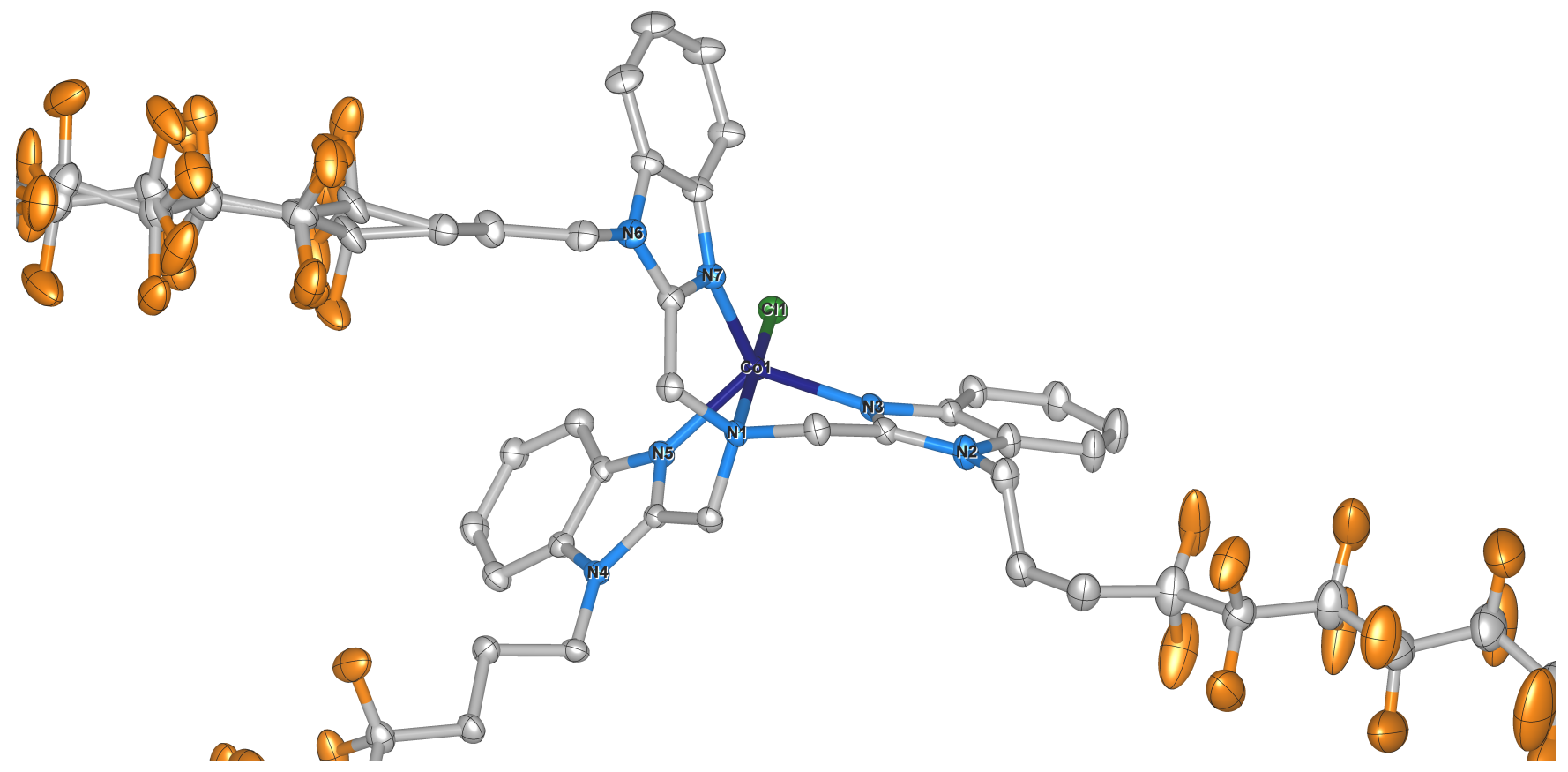

Figure S21. The coordination center of $\mathbf{C o}-\left(\mathbf{B i m C}_{3} \mathbf{F}_{8}\right)_{3}$. Hydrogen atoms, counter ions and solvents are omitted for clarity. Cobalt (dark blue), nitrogen (blue), carbon (gray), chloride (green) and fluorine (orange). 
Table S5. Crystal Data and Structure Refinement Details for Co- $\left(\mathbf{B i m C}_{3} \mathbf{F}_{8}\right)_{3}$.

\begin{tabular}{|c|c|}
\hline Identification code & $\mathrm{Co}-\left(\mathrm{BimC}_{3} \mathrm{~F}_{8}\right)_{3}$ \\
\hline Empirical formula & $\mathrm{C}_{69} \mathrm{H}_{63} \mathrm{Cl}_{2} \mathrm{CoF}_{51} \mathrm{~N}_{7} \mathrm{O}_{5}$ \\
\hline Formula weight & 2169.10 \\
\hline Temperature/K & $200(2)$ \\
\hline Crystal system & monoclinic \\
\hline Space group & $\mathrm{P} 2_{1} / \mathrm{c}$ \\
\hline $\mathrm{a} / \AA ̊ \AA$ & $24.5991(11)$ \\
\hline $\mathrm{b} / \AA$ & $14.5605(6)$ \\
\hline$c / \AA ̊$ & $25.6393(11)$ \\
\hline$\alpha /^{\circ}$ & 90 \\
\hline$\beta /{ }^{\circ}$ & $106.1210(10)$ \\
\hline$\gamma /{ }^{\circ}$ & 90 \\
\hline Volume $/ \AA^{3}$ & $8822.2(7)$ \\
\hline $\mathrm{Z}$ & 4 \\
\hline$\rho$ calcg $/ \mathrm{cm}^{3}$ & 1.634 \\
\hline$\mu / \mathrm{mm}^{-1}$ & 0.414 \\
\hline $\mathrm{F}(000)$ & 4348.0 \\
\hline Crystal size $/ \mathrm{mm}^{3}$ & $0.56 \times 0.44 \times 0.24$ \\
\hline Radiation & $\operatorname{MoK} \alpha(\lambda=0.71073)$ \\
\hline $2 \Theta$ range for data collection $/^{\circ}$ & 4.31 to 50.052 \\
\hline Index ranges & $-29 \leq \mathrm{h} \leq 29,-17 \leq \mathrm{k} \leq 17,-30 \leq 1 \leq 30$ \\
\hline Reflections collected & 439740 \\
\hline Independent reflections & $15563\left[\mathrm{R}_{\text {int }}=0.0758, \mathrm{R}_{\text {sigma }}=0.0183\right]$ \\
\hline Data/restraints/parameters & $15563 / 1116 / 1397$ \\
\hline Goodness-of-fit on $\mathrm{F}^{2}$ & 1.057 \\
\hline Final R indexes $[\mathrm{I}>=2 \sigma(\mathrm{I})]$ & $\mathrm{R}_{1}=0.0871, \mathrm{wR}_{2}=0.2466$ \\
\hline Final $\mathrm{R}$ indexes [all data ] & $\mathrm{R}_{1}=0.1033, \mathrm{wR}_{2}=0.2662$ \\
\hline Largest diff. peak/hole / e $\AA^{-3}$ & $1.03 /-0.78$ \\
\hline
\end{tabular}


Table S6. Selected bond distances and angles of $\mathbf{C o -}\left(\mathbf{B i m C}_{3} \mathbf{F}_{8}\right)_{3}$.

\begin{tabular}{|c|c|c|c|}
\hline \multicolumn{5}{|c|}{ Bond Length/A } \\
\hline Co1-N1 & $2.332(3)$ & Co1-N3 & $2.033(4)$ \\
\hline Co1-N5 & $2.048(3)$ & Co1-N7 & \\
\hline Co1-Cl1 & $2.2975(11)$ & N3-Co1-Cl1 & $103.34(10)$ \\
\hline \multicolumn{2}{|c|}{ Angle $^{\circ}$} \\
\hline Cl1-Co1-N1 & $177.18(9)$ & N3-Co1-N5 & $119.90(14)$ \\
\hline N3-Co1-N1 & $76.37(13)$ & N5-Co1-N1 & $75.24(13)$ \\
\hline N5-Co1-Cl1 & $102.66(10)$ & N7-Co1-N1 & $75.82(13)$ \\
\hline N7-Co1-Cl1 & $106.77(10)$ & N7-Co1-N5 & $109.15(14)$ \\
\hline N7-Co1-N3 & $113.45(14)$ &
\end{tabular}




\section{NMR Spectra}

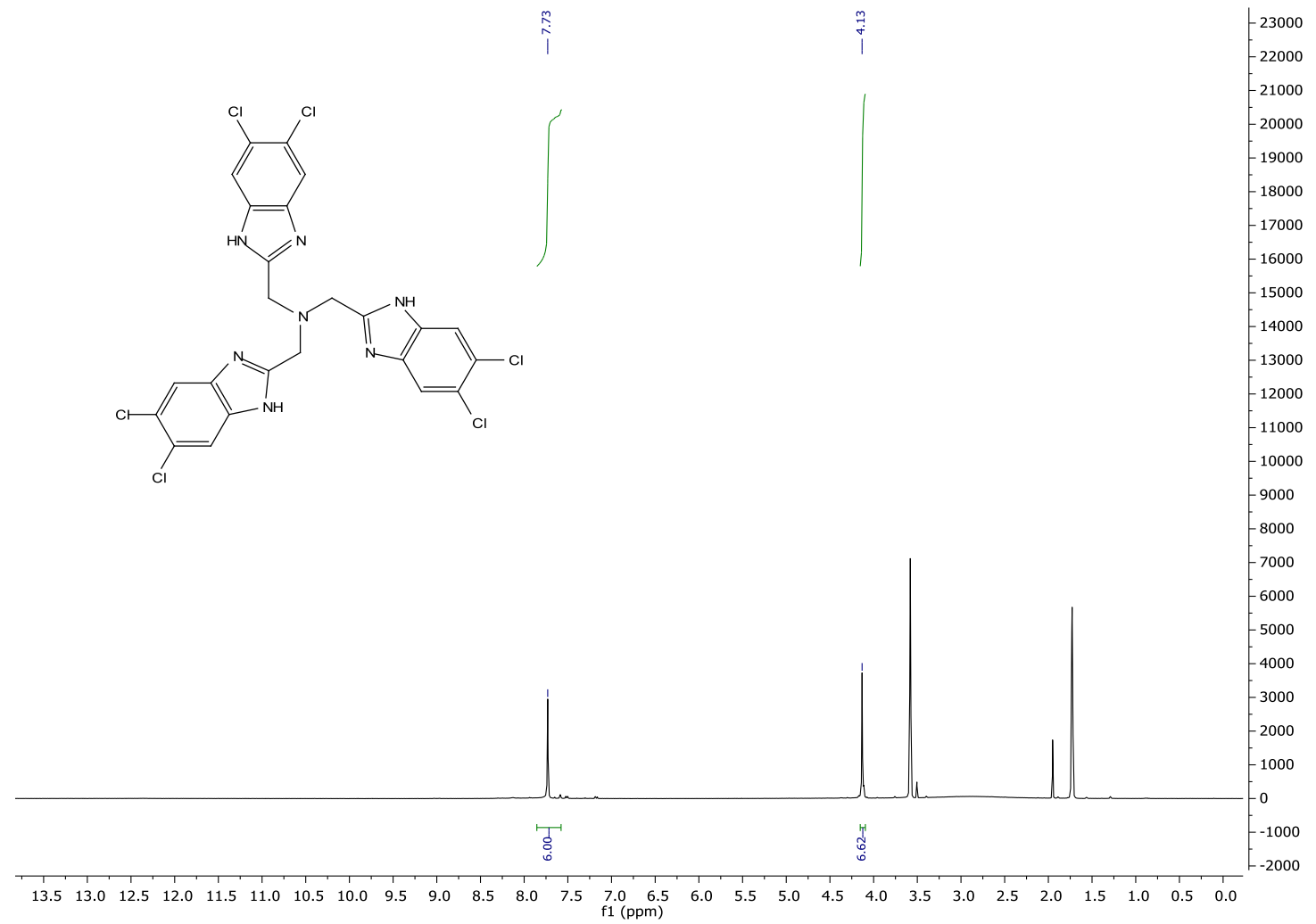

Figure S22. ${ }^{1} \mathrm{H}$ NMR of $\left(\mathbf{B i m H} / \mathbf{C l}_{2}\right)_{3}$ in THF- $d 8$.

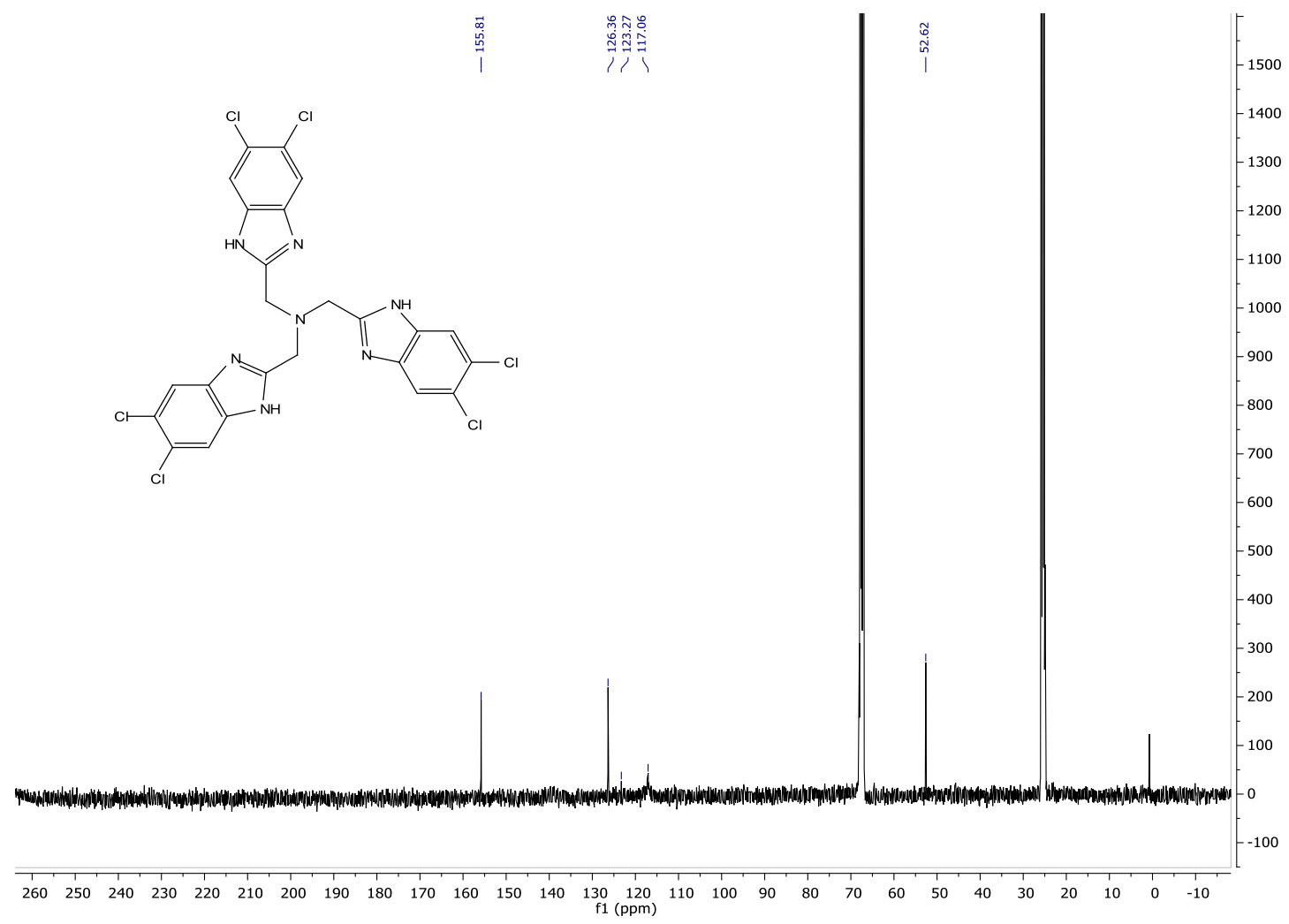

Figure S23. ${ }^{13} \mathrm{C}$ NMR of $\left(\mathbf{B i m H} / \mathbf{C l}_{2}\right)_{3}$ in THF- $d 8$. 


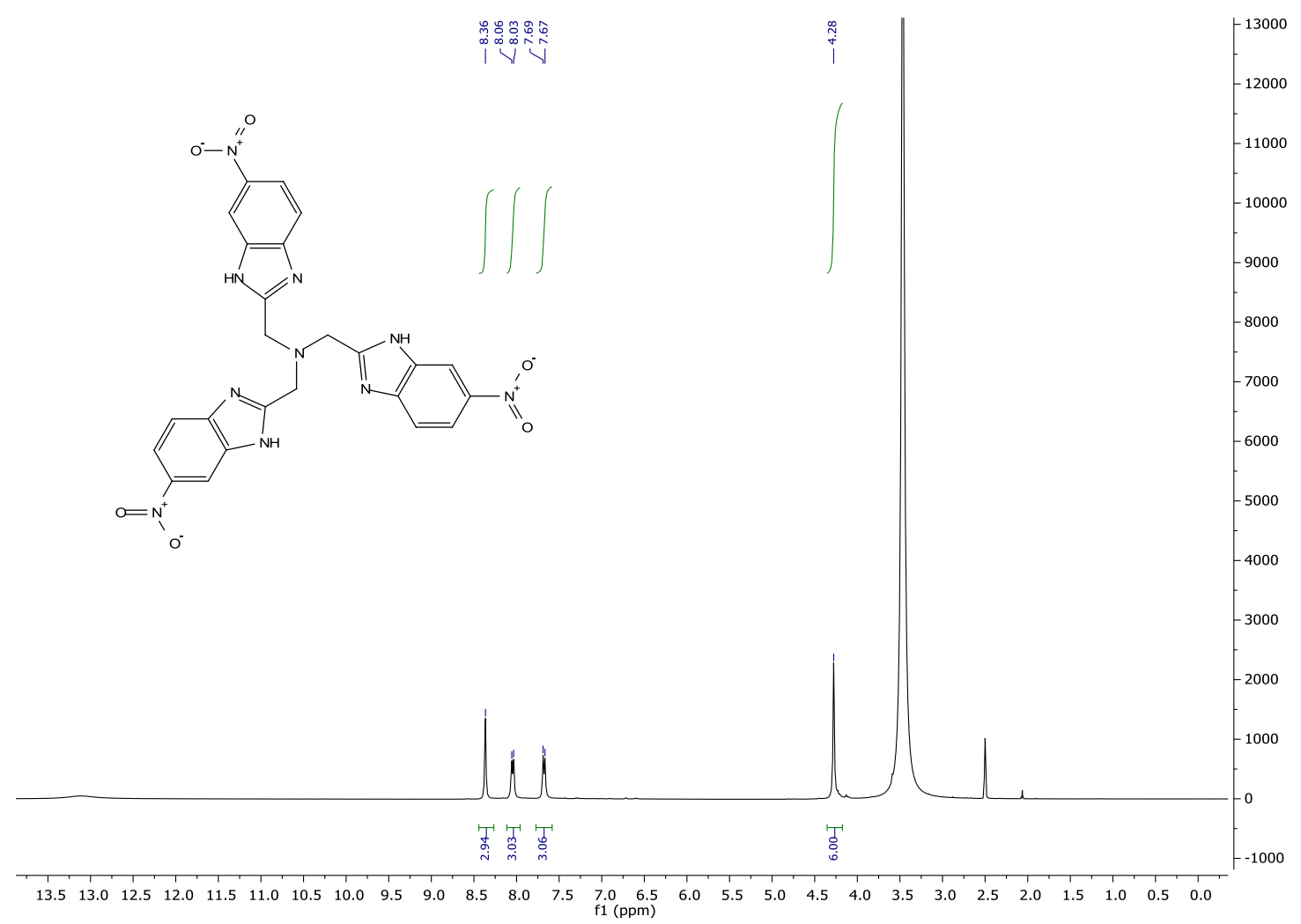

Figure S24. ${ }^{1} \mathrm{H}$ NMR of $\left(\mathrm{BimH} / \mathrm{NO}_{2}\right)_{3}$ in DMSO-d6.

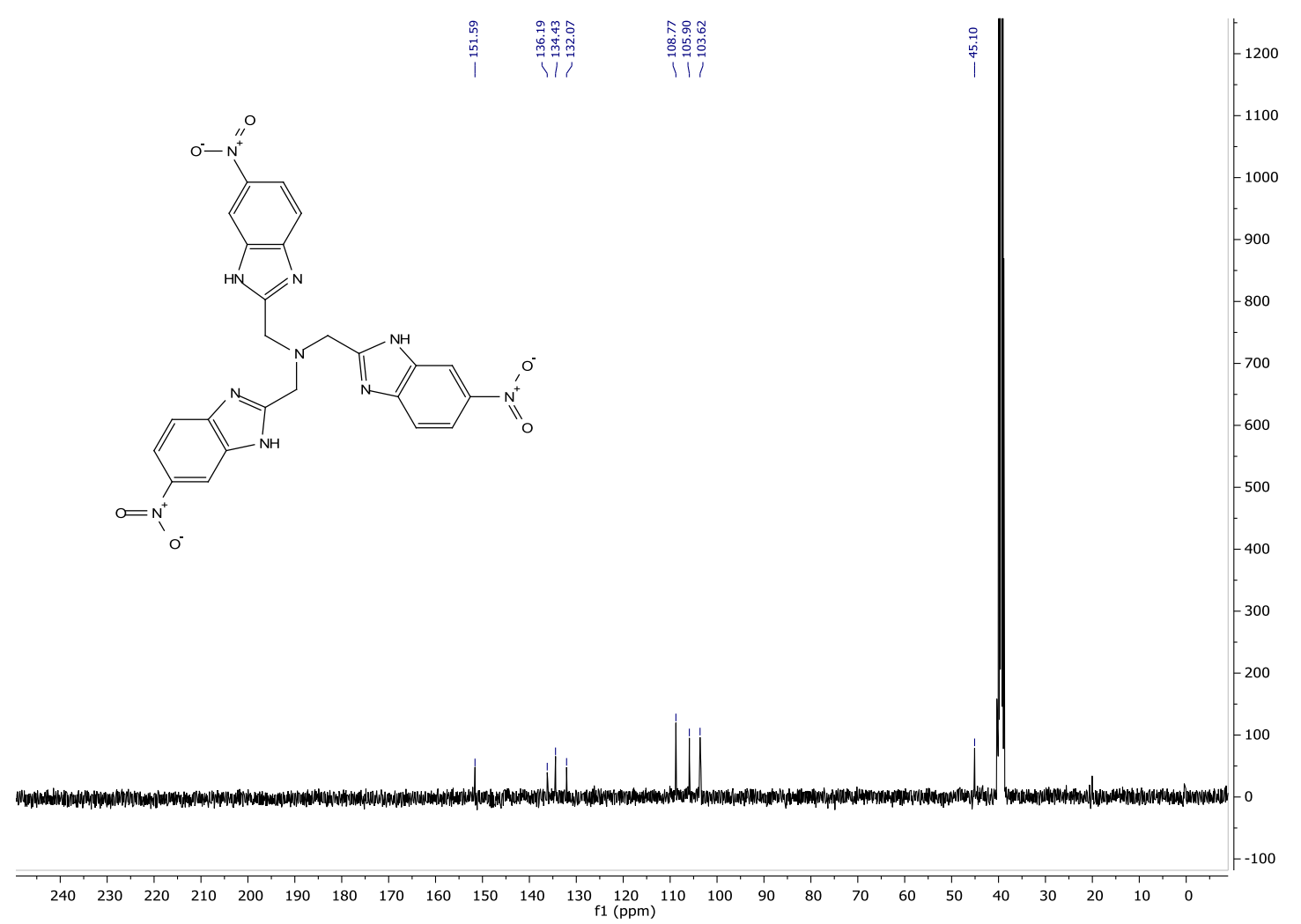

Figure S25. ${ }^{13} \mathrm{C}$ NMR of $\left(\mathbf{B i m H} / \mathbf{N O}_{2}\right)_{3}$ in DMSO-d6. 


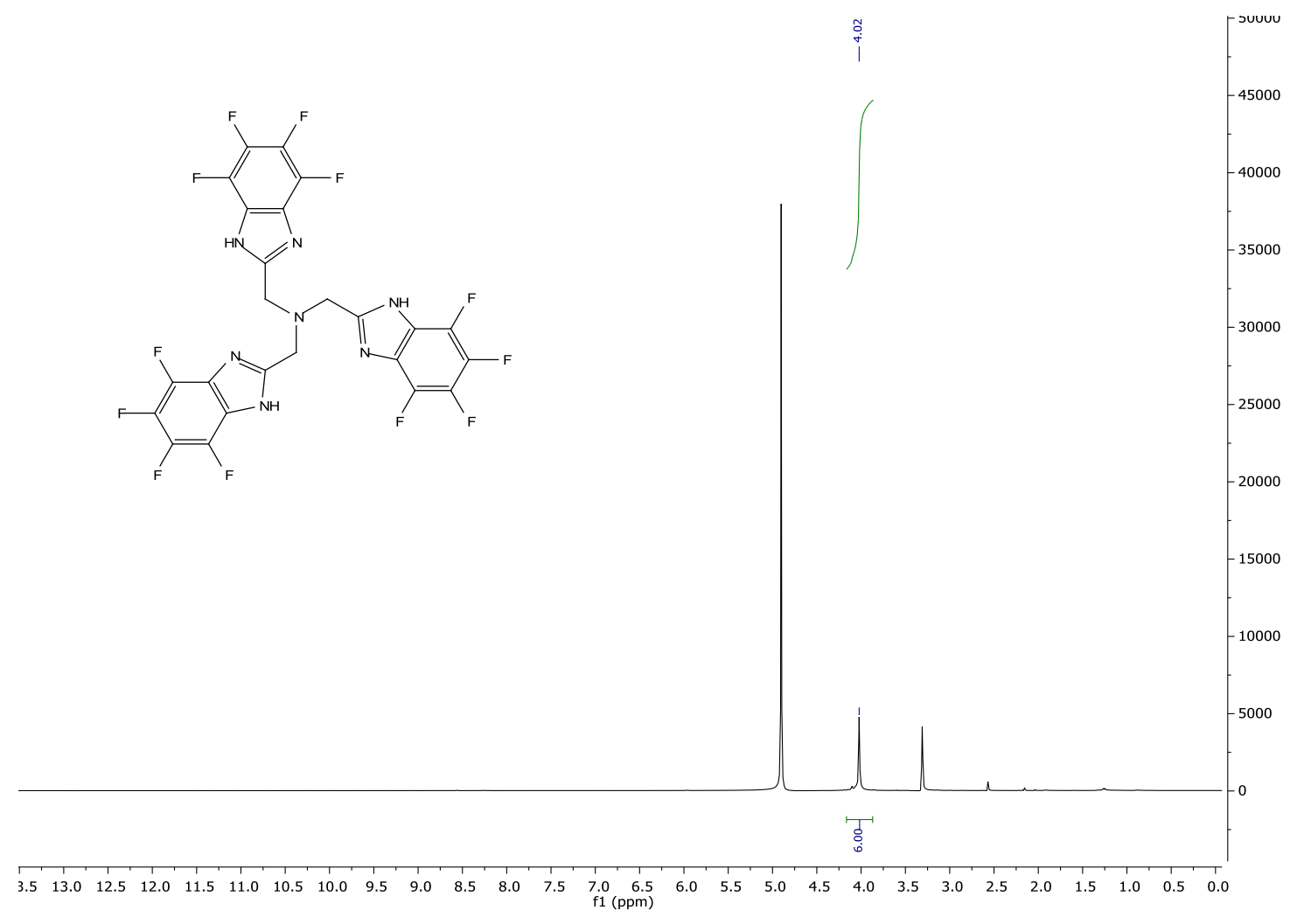

Figure S26. ${ }^{1} \mathrm{H}$ NMR of $\left(\mathbf{B i m H} / \mathbf{F}_{4}\right)_{3}$ in methanol- $d_{4}$.

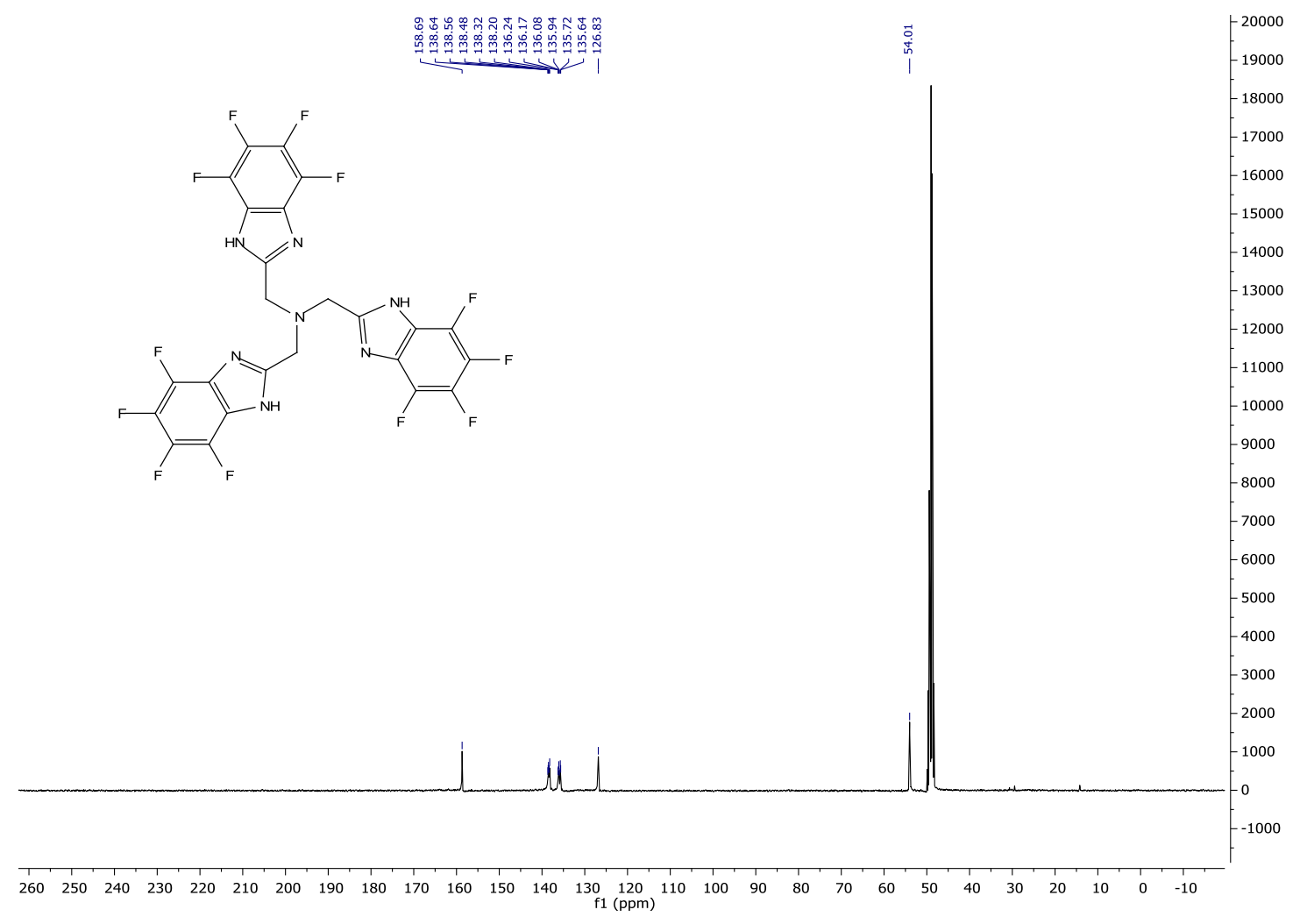

Figure S27. ${ }^{13} \mathrm{C}$ NMR of $\left(\mathbf{B i m H} / \mathbf{F}_{4}\right)_{3}$ in methanol- $d_{4}$. 


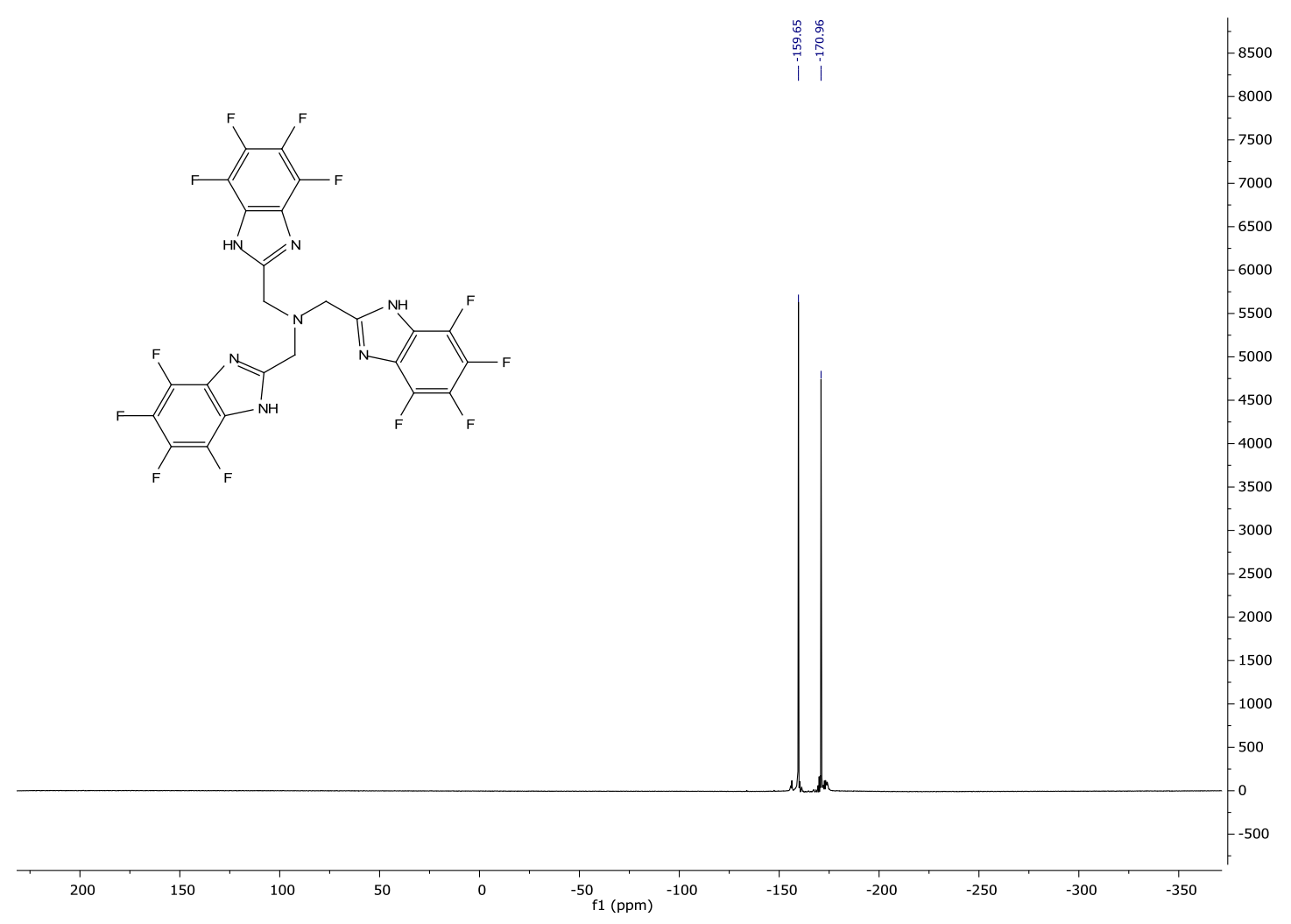

Figure S28. ${ }^{19} \mathrm{~F}$ NMR of $\left(\mathbf{B i m H} / \mathbf{F}_{4}\right)_{3}$ in methanol- $d_{4}$.

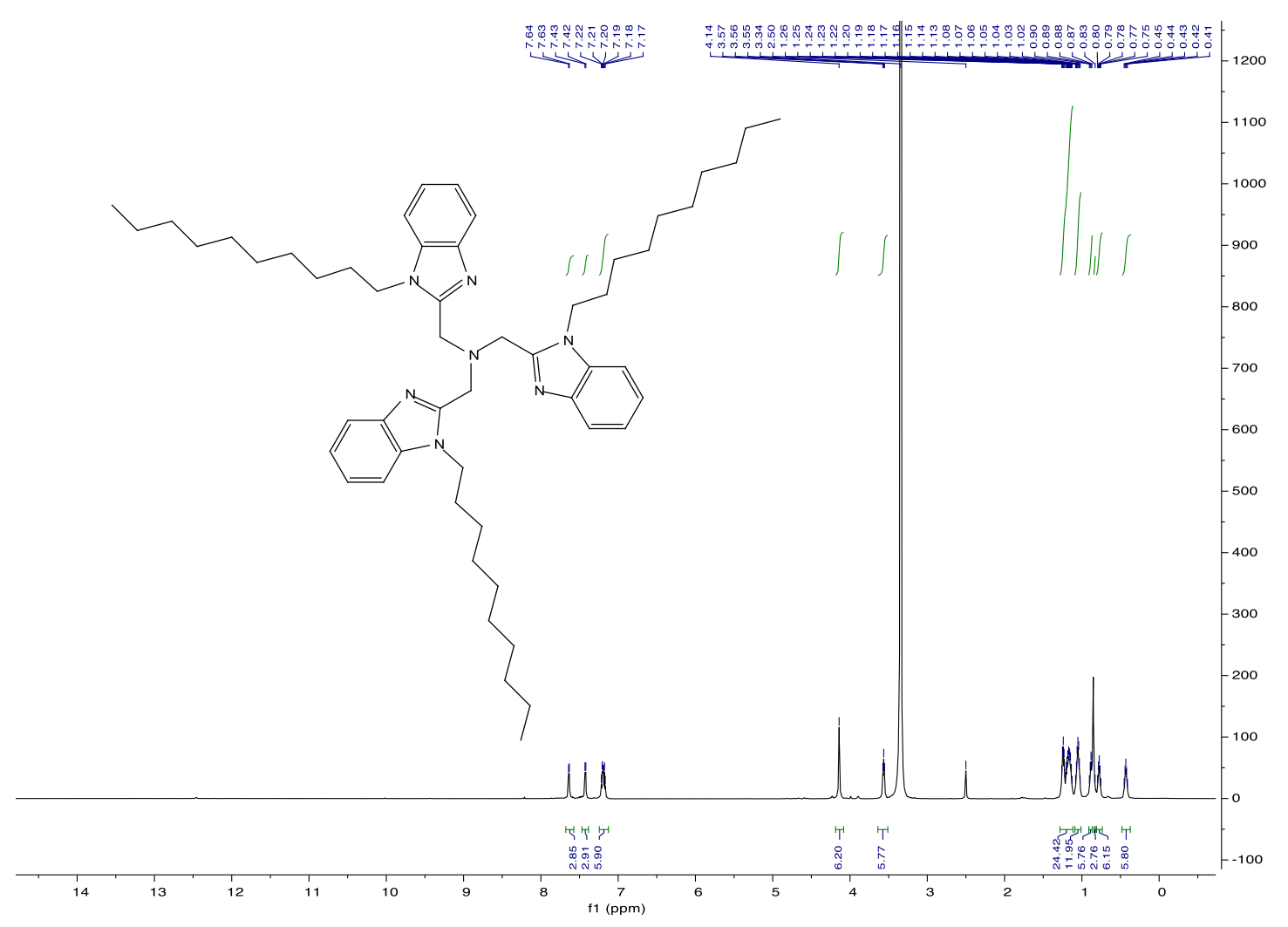

Figure S29. ${ }^{1} \mathrm{H}$ NMR of $\left(\operatorname{BimC}_{10} \mathbf{H}\right)_{3}$ in DMSO-d6. 


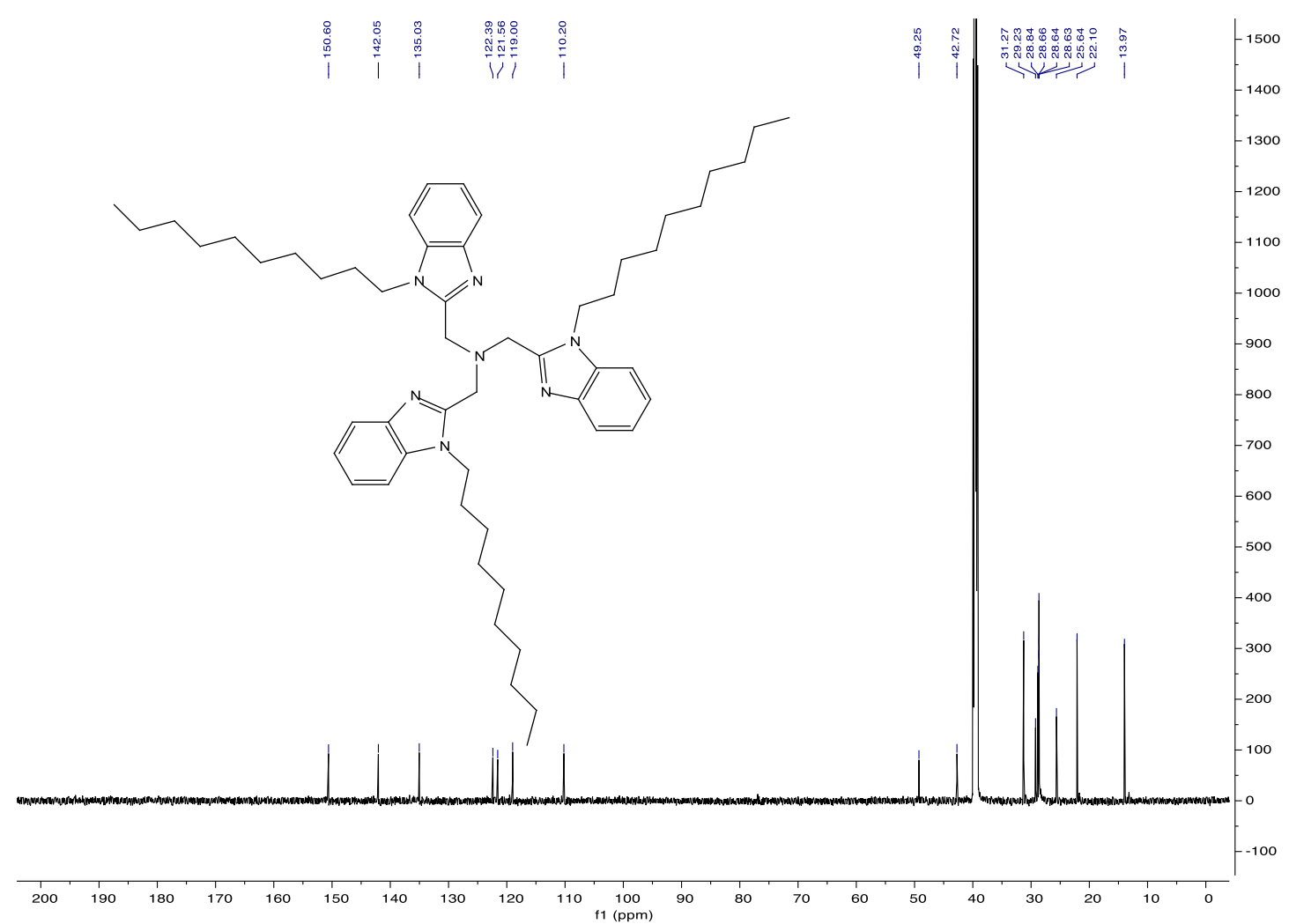

Figure S30. ${ }^{13} \mathrm{C}$ NMR of $\left(\mathbf{B i m C}_{10} \mathbf{H}\right)_{3}$ in DMSO-d6.

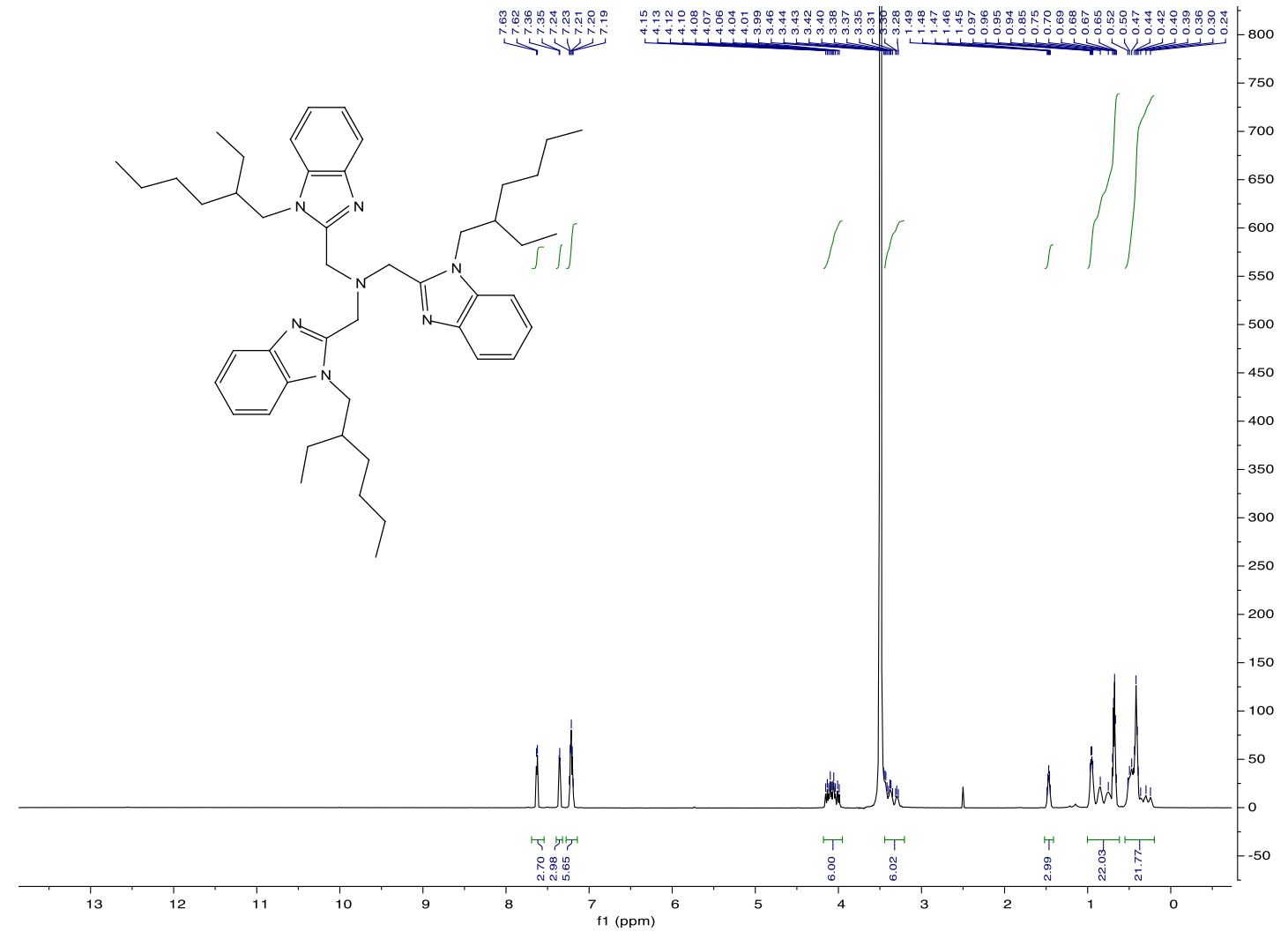

Figure S31. ${ }^{1} \mathrm{H}$ NMR of $\left(\mathbf{B i m C}_{6+2} \mathbf{H}\right)_{3}$ in DMSO-d6. 


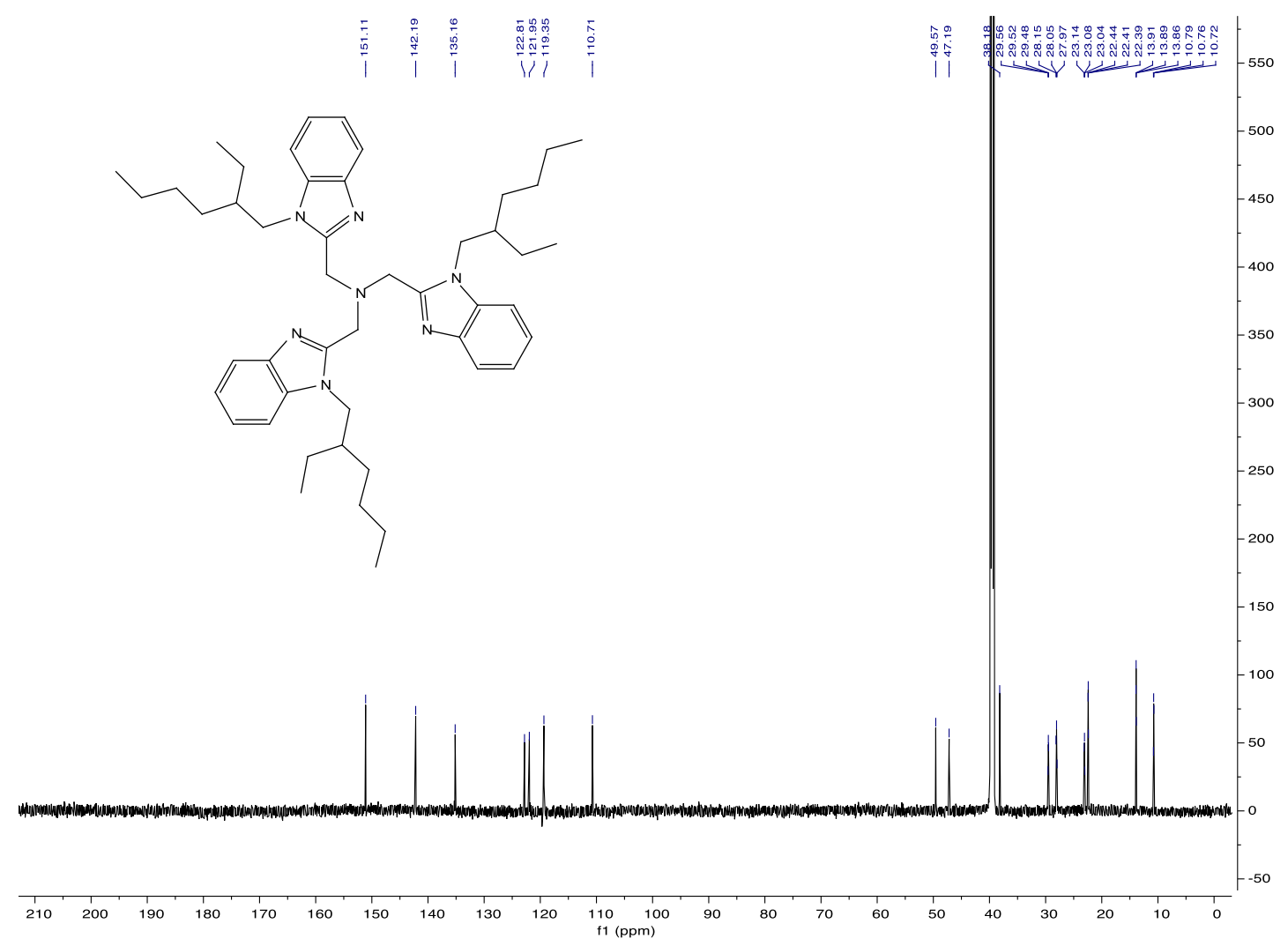

Figure S32. ${ }^{13} \mathrm{C}$ NMR of $\left(\mathrm{BimC}_{6+2} \mathbf{H}\right)_{3}$ in DMSO-d6.

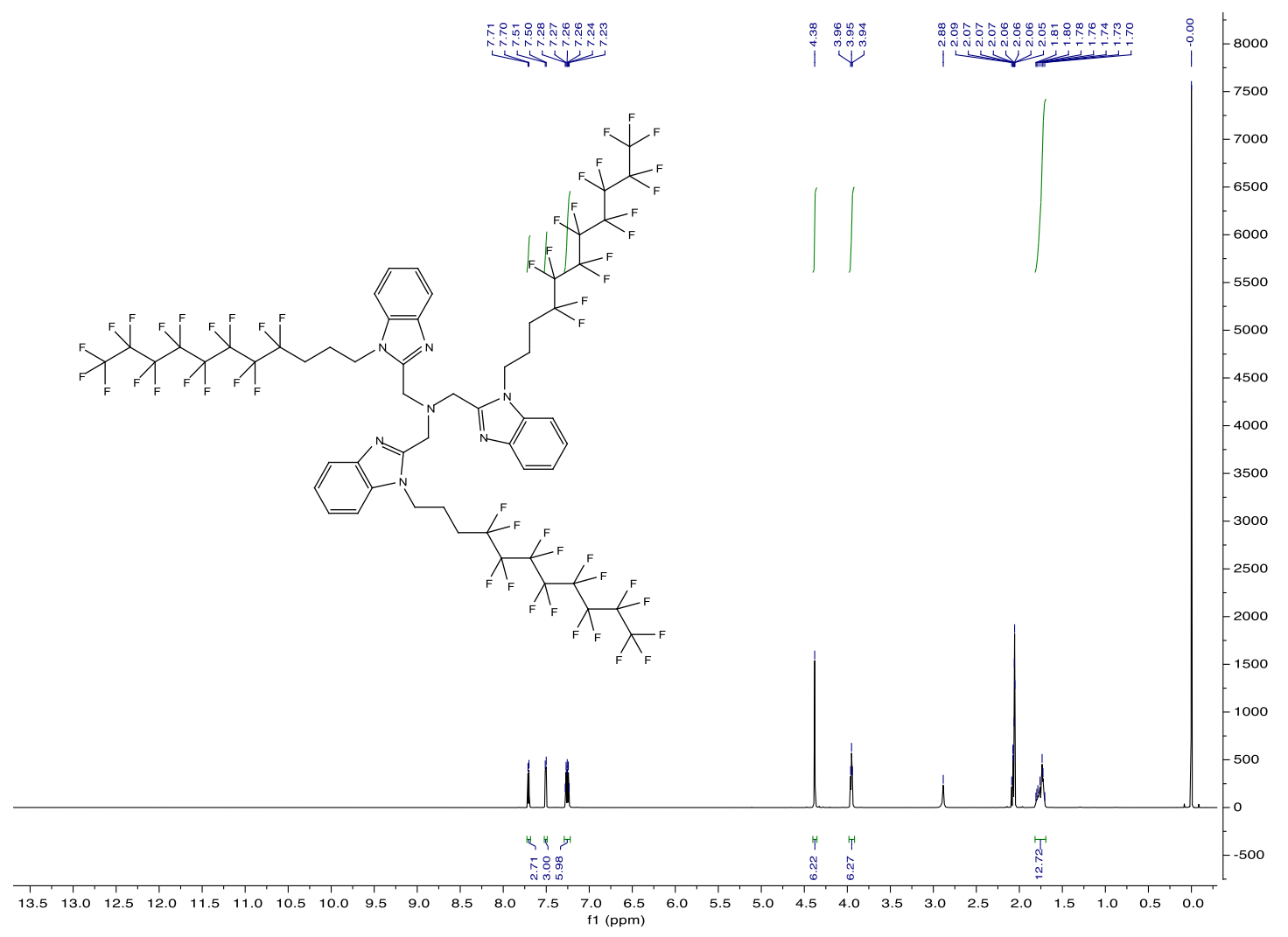

Figure S33. ${ }^{1} \mathrm{H}$ NMR of $\left(\mathbf{B i m C}_{3} \mathbf{F}_{8}\right)_{3}$ in $\left(\mathrm{CD}_{3}\right)_{2} \mathrm{CO}$. 


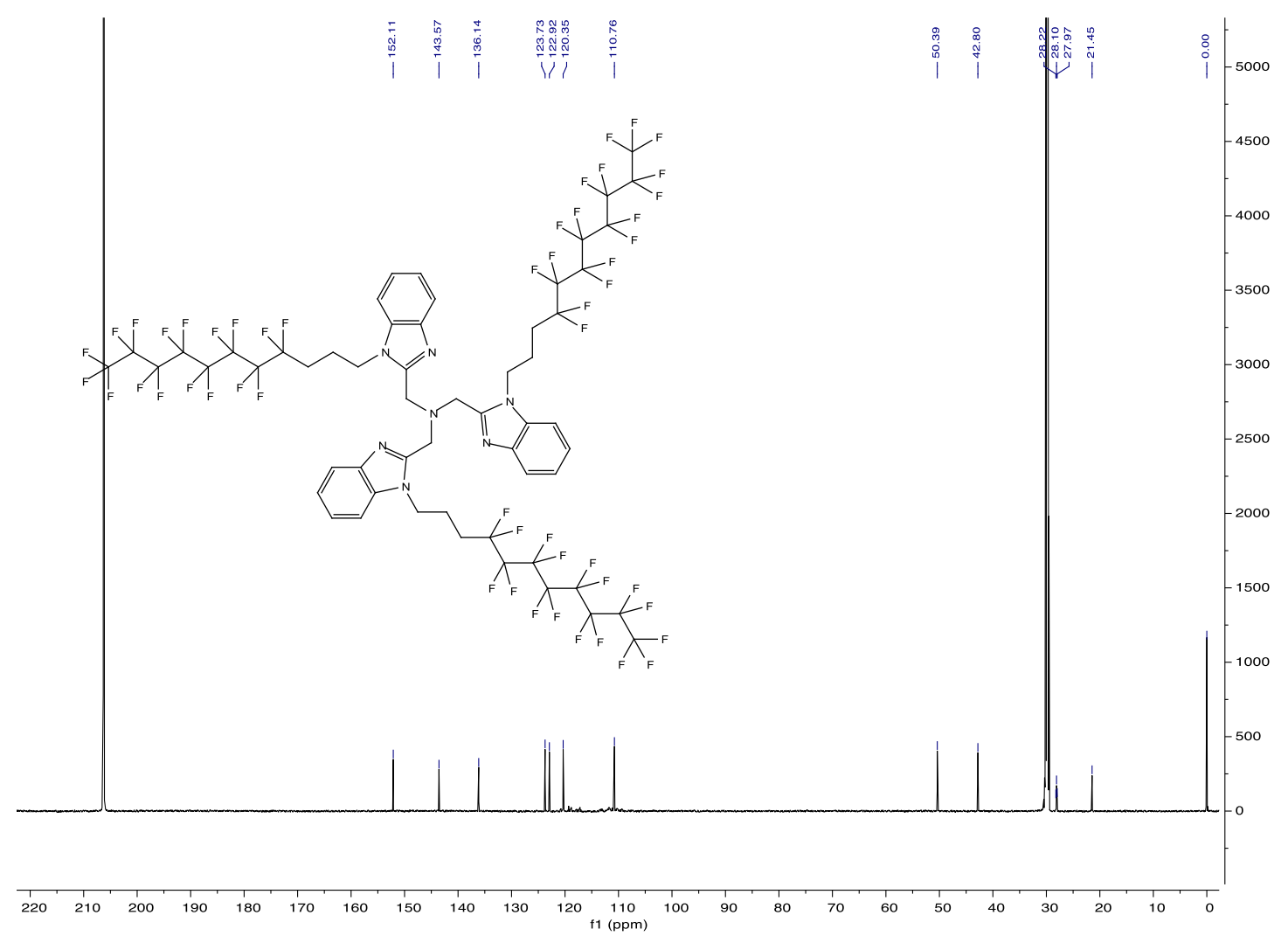

Figure S34. ${ }^{13} \mathrm{C}$ NMR of $\left(\mathbf{B i m C}_{3} \mathbf{F}_{8}\right)_{3}$ in $\left(\mathrm{CD}_{3}\right)_{2} \mathrm{CO}$.

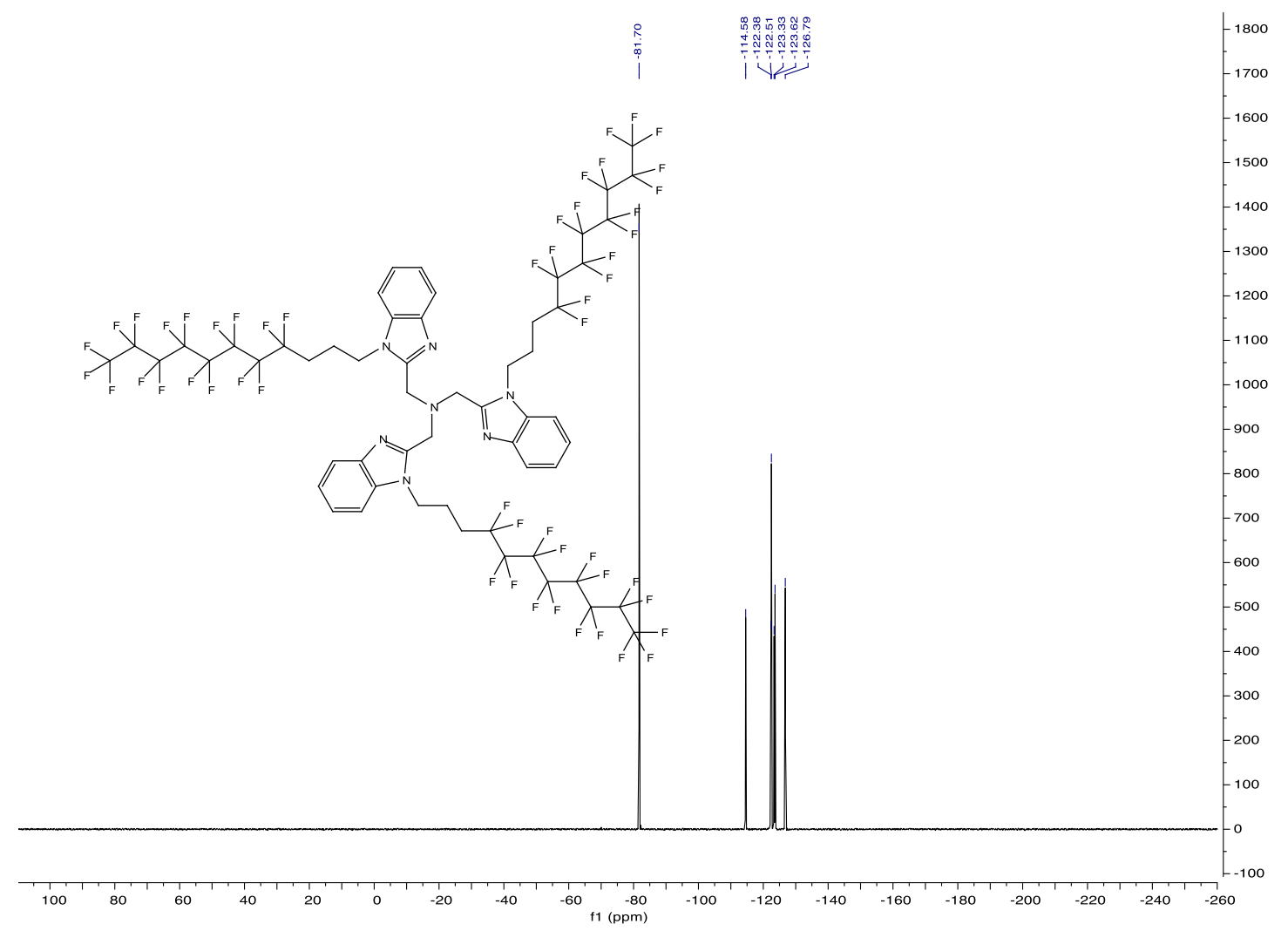

Figure S35. ${ }^{19} \mathrm{~F}$ NMR of $\left(\mathbf{B i m C}_{3} \mathbf{F}_{8}\right)_{3}$ in $\left(\mathrm{CD}_{3}\right)_{2} \mathrm{CO}$. 


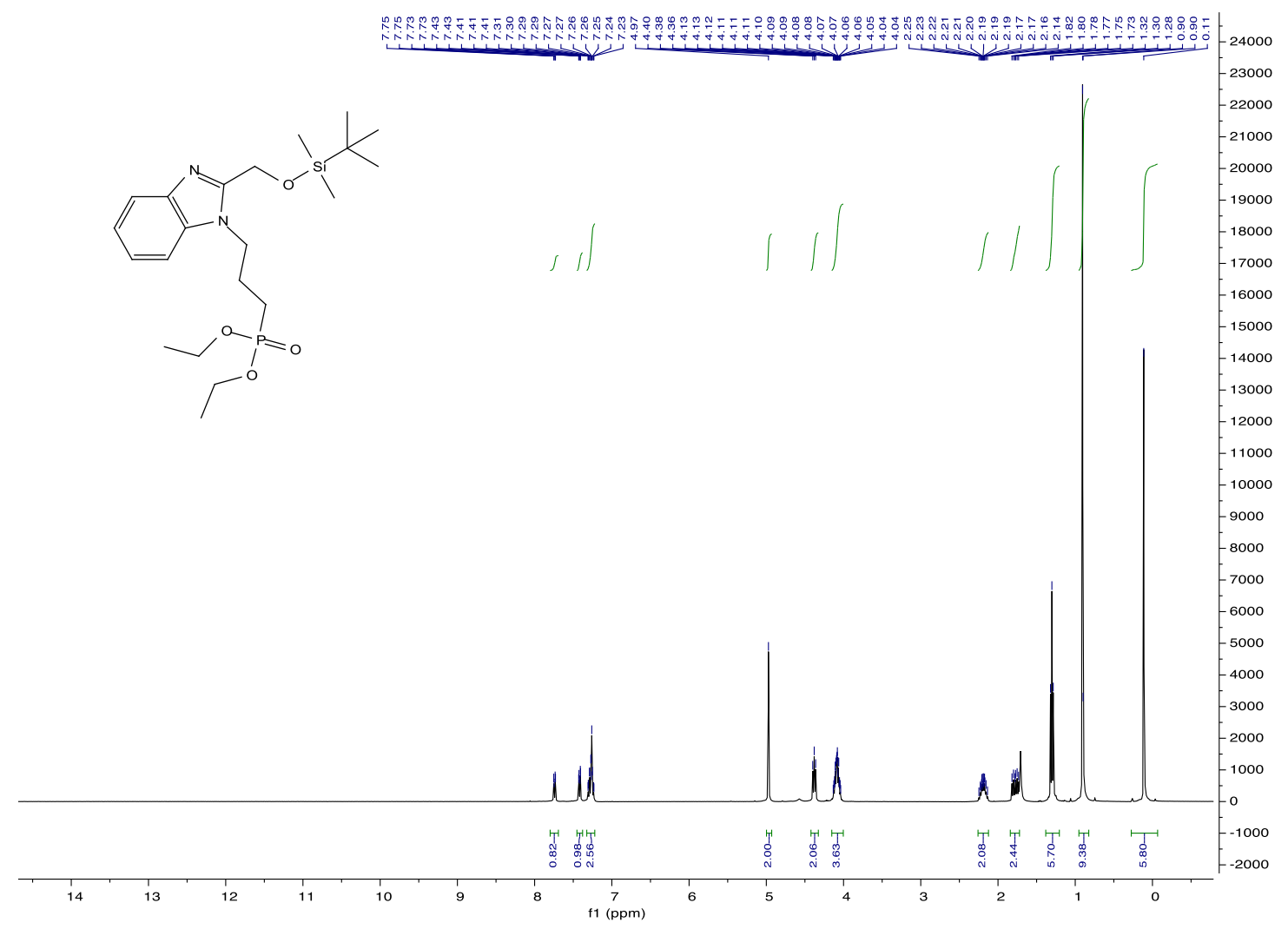

Figure S36. ${ }^{1} \mathrm{H}$ NMR of $\left(\mathbf{B i m C}_{3} \mathbf{P E}\right) \mathbf{C O S i}$ in $\mathrm{CDCl}_{3}$.

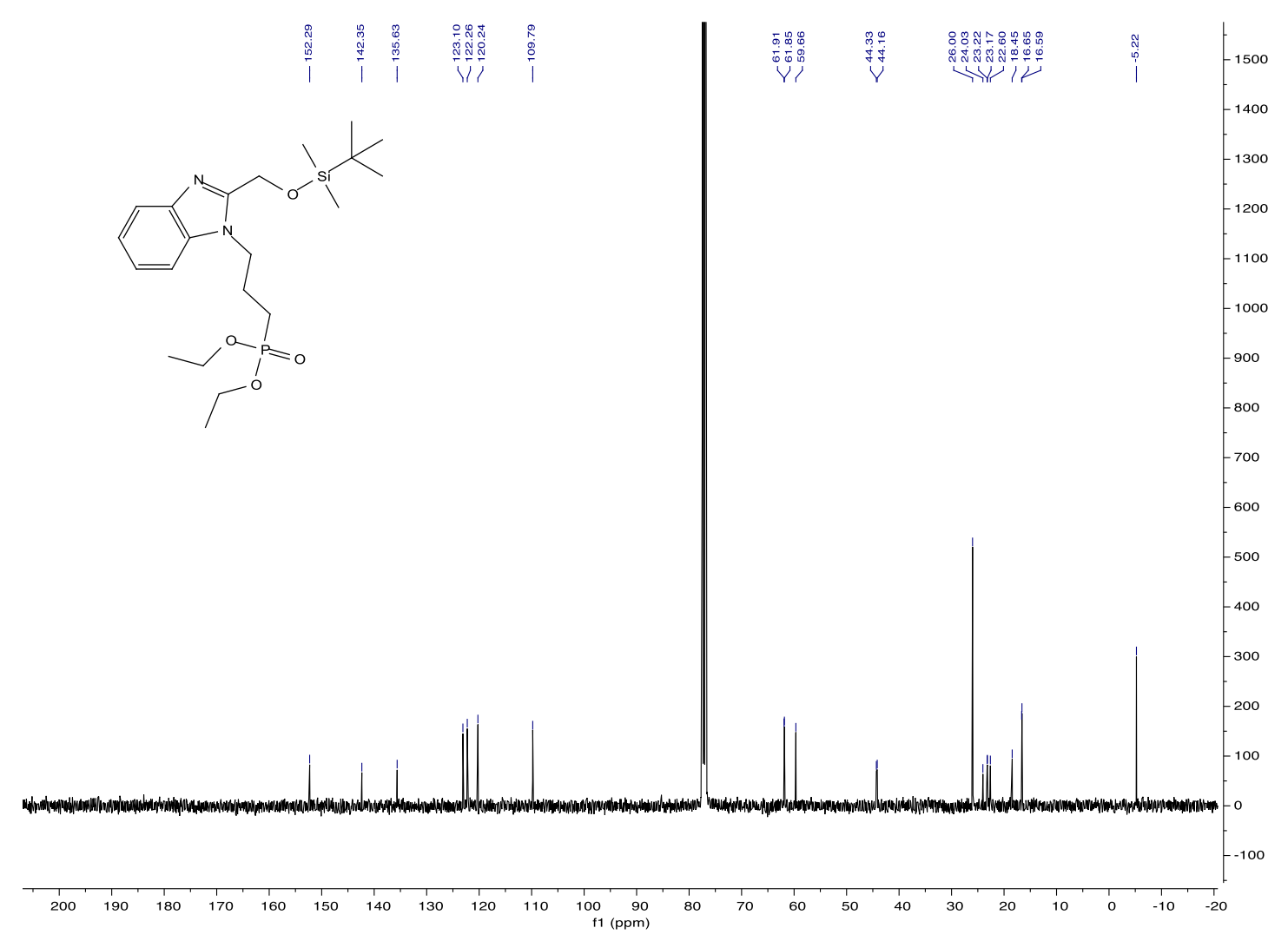

Figure S37. ${ }^{13} \mathrm{C}$ NMR of $\left(\right.$ BimC $\left._{3} \mathbf{P E}\right) \mathbf{C O S i}$ in $\mathrm{CDCl}_{3}$. 


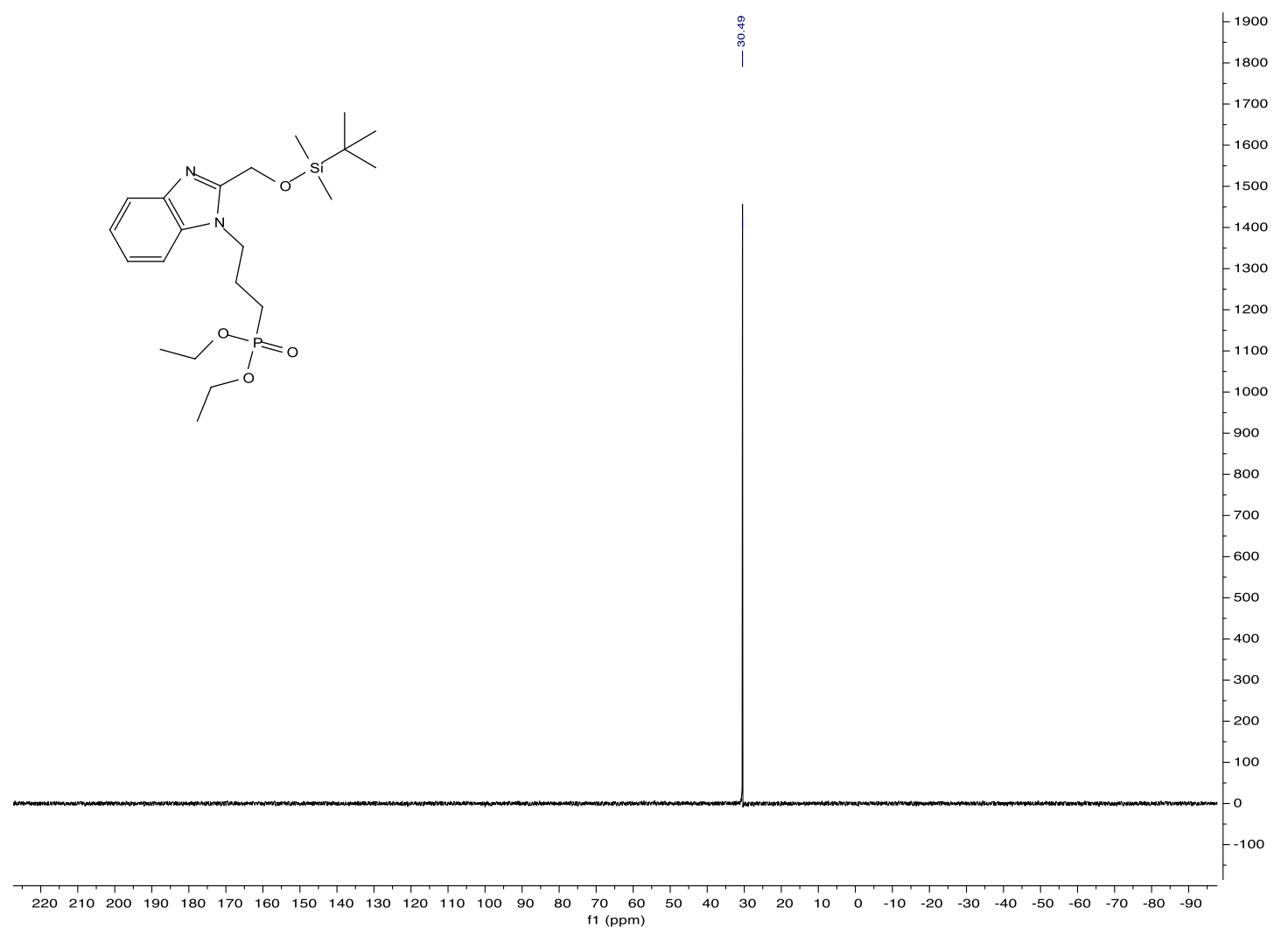

Figure S38. ${ }^{31} \mathrm{P}$ NMR of $\left(\mathbf{B i m C}_{3} \mathbf{P E}\right) \mathbf{C O S i}$ in $\mathrm{CDCl}_{3}$.

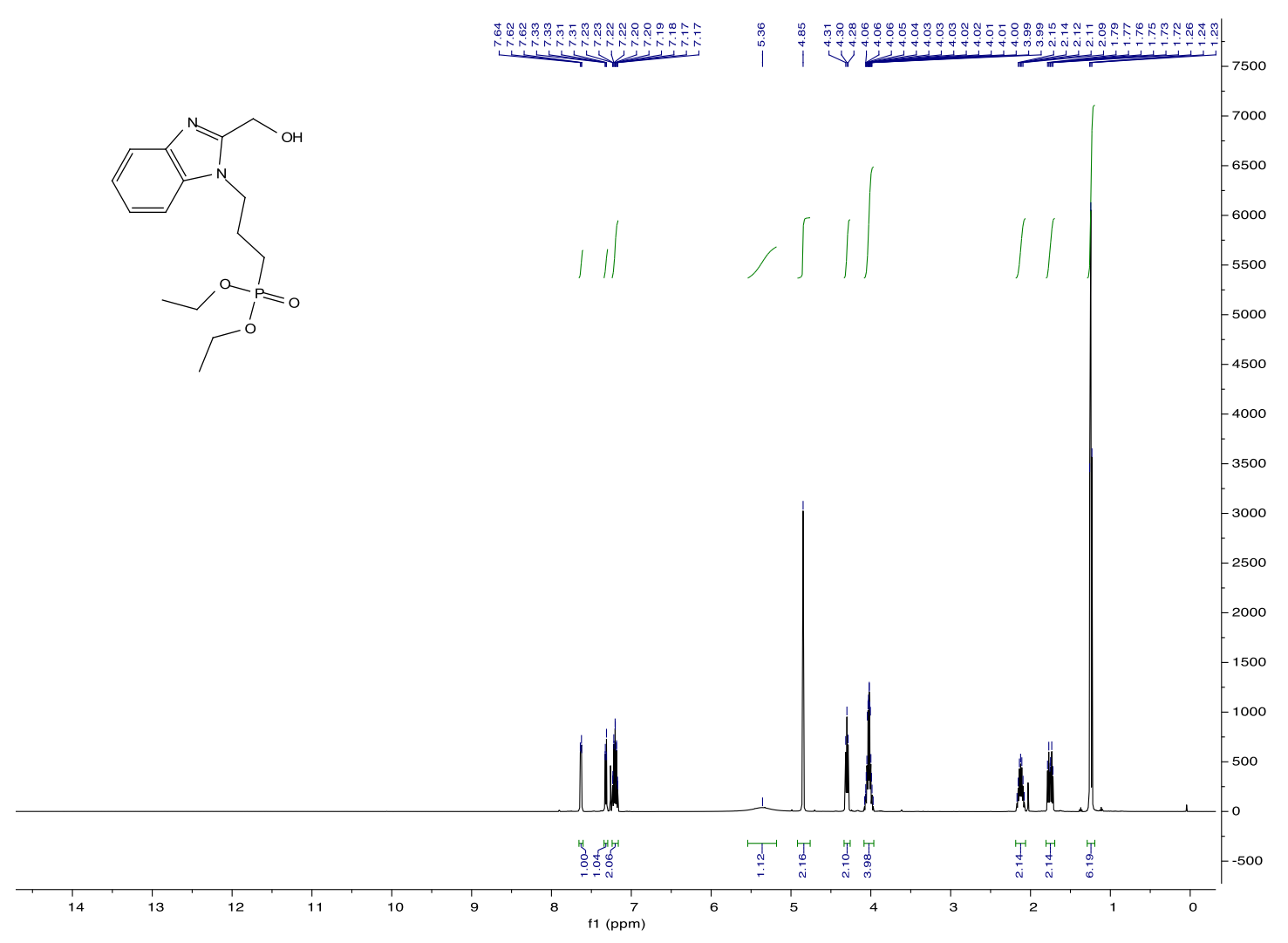

Figure S39. ${ }^{1} \mathrm{H}$ NMR of $\left(\mathbf{B i m C}_{3} \mathbf{P E}\right) \mathbf{C O H}$ in $\mathrm{CDCl}_{3}$. 


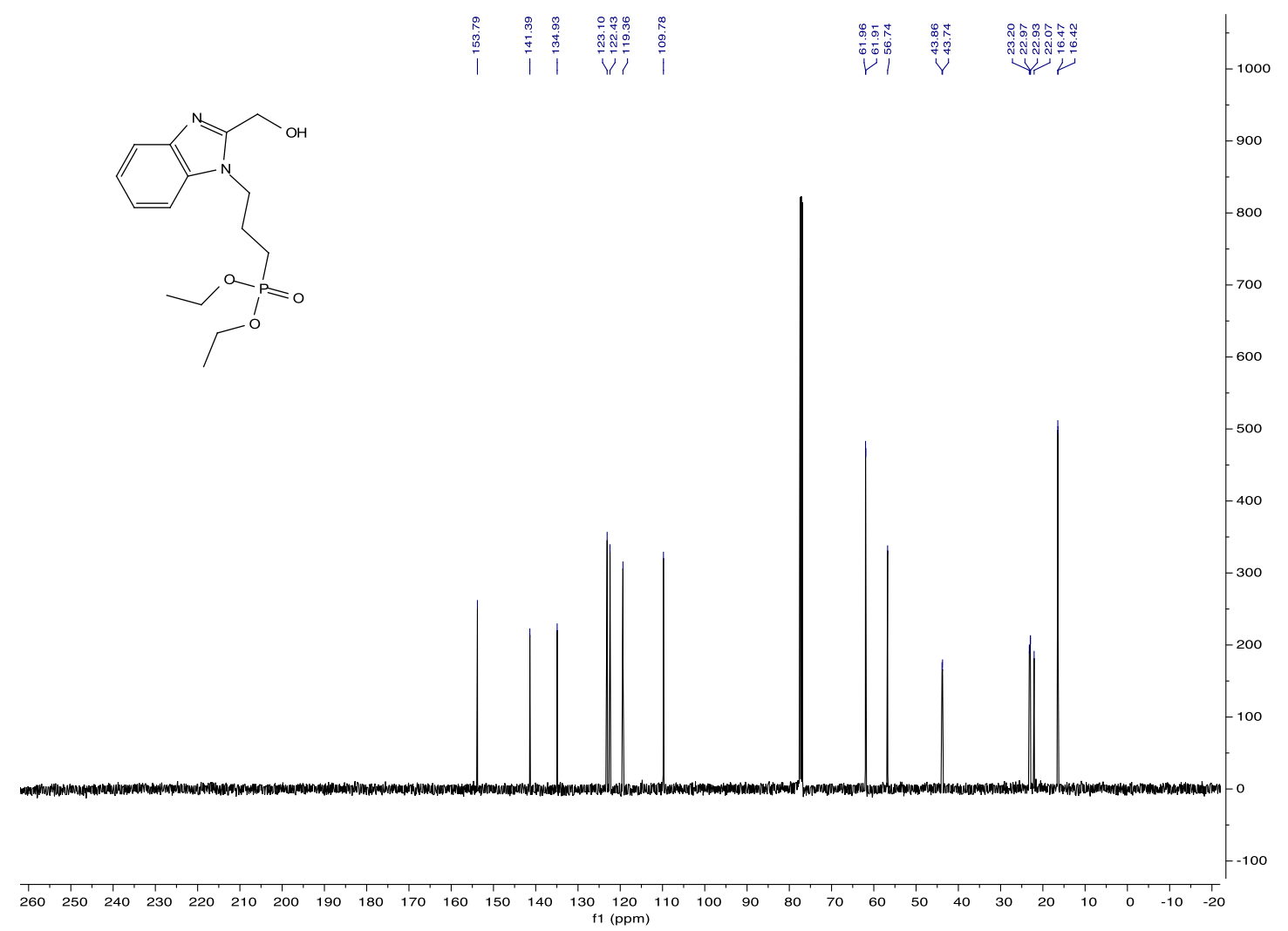

Figure S40. ${ }^{13} \mathrm{C}$ NMR of $\left(\mathbf{B i m C}_{3} \mathbf{P E}\right) \mathbf{C O H}$ in $\mathrm{CDCl}_{3}$.

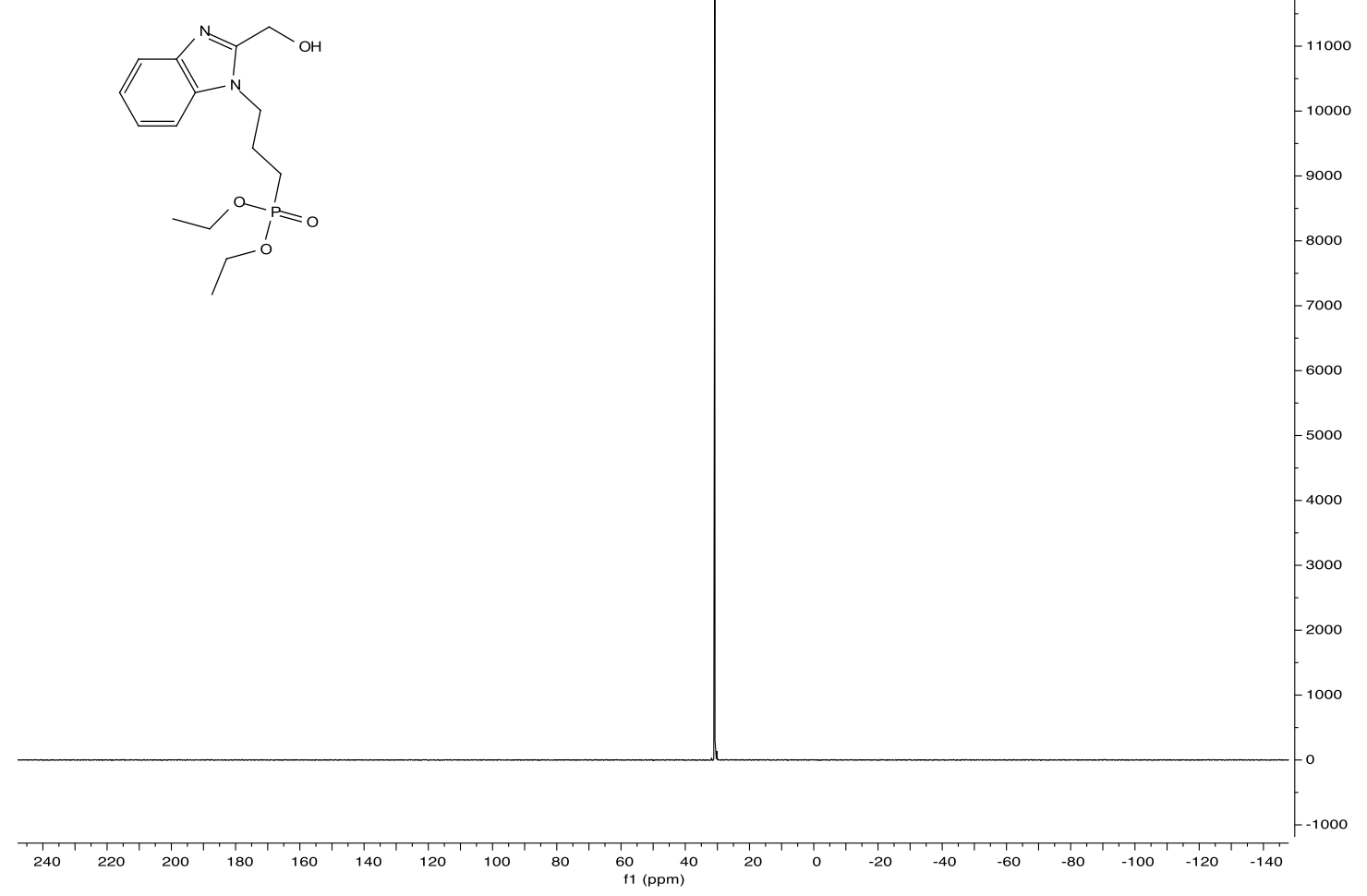

Figure S41. ${ }^{31} \mathrm{P}$ NMR of $\left(\mathbf{B i m C}_{3} \mathbf{P E}\right) \mathbf{C O H}$ in $\mathrm{CDCl}_{3}$. 


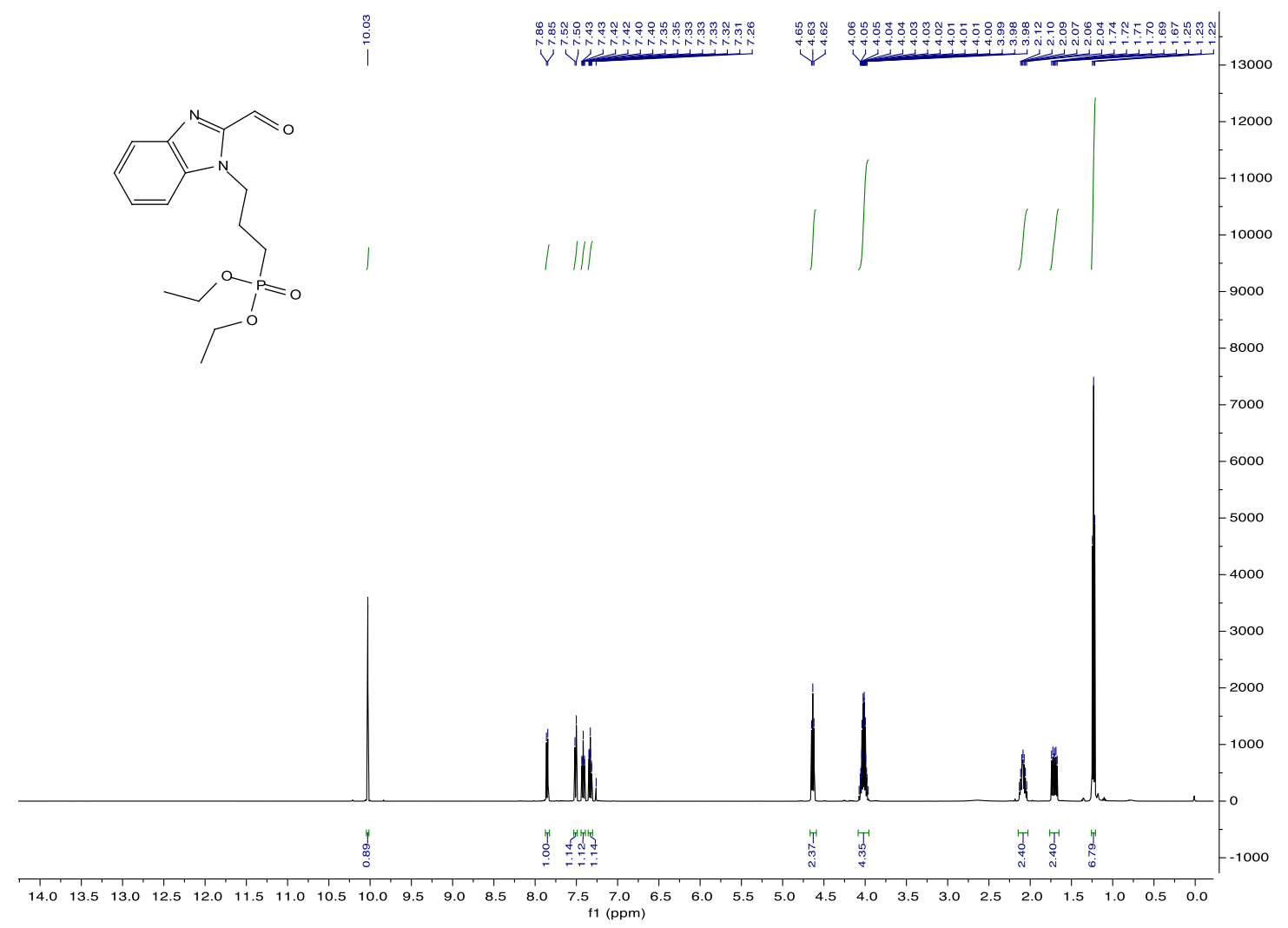

Figure S42. ${ }^{1} \mathrm{H} \mathrm{NMR}$ of $\left(\mathbf{B i m C}_{3} \mathbf{P E}\right) \mathbf{C H O}$ in $\mathrm{CDCl}_{3}$.

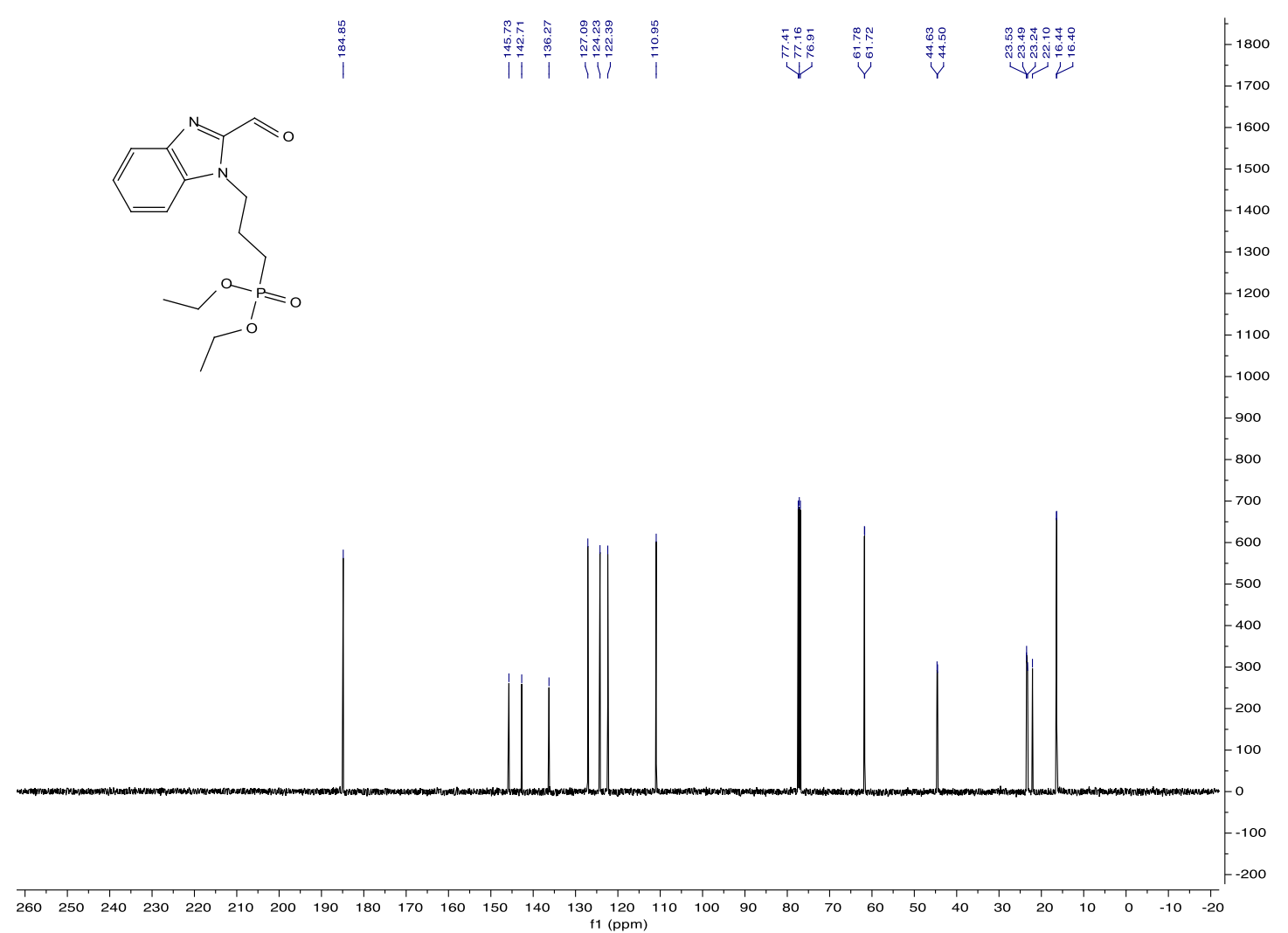

Figure S43. ${ }^{13} \mathrm{C}$ NMR of $\left(\mathrm{BimC}_{3} \mathbf{P E}\right) \mathbf{C H O}$ in $\mathrm{CDCl}_{3}$. 


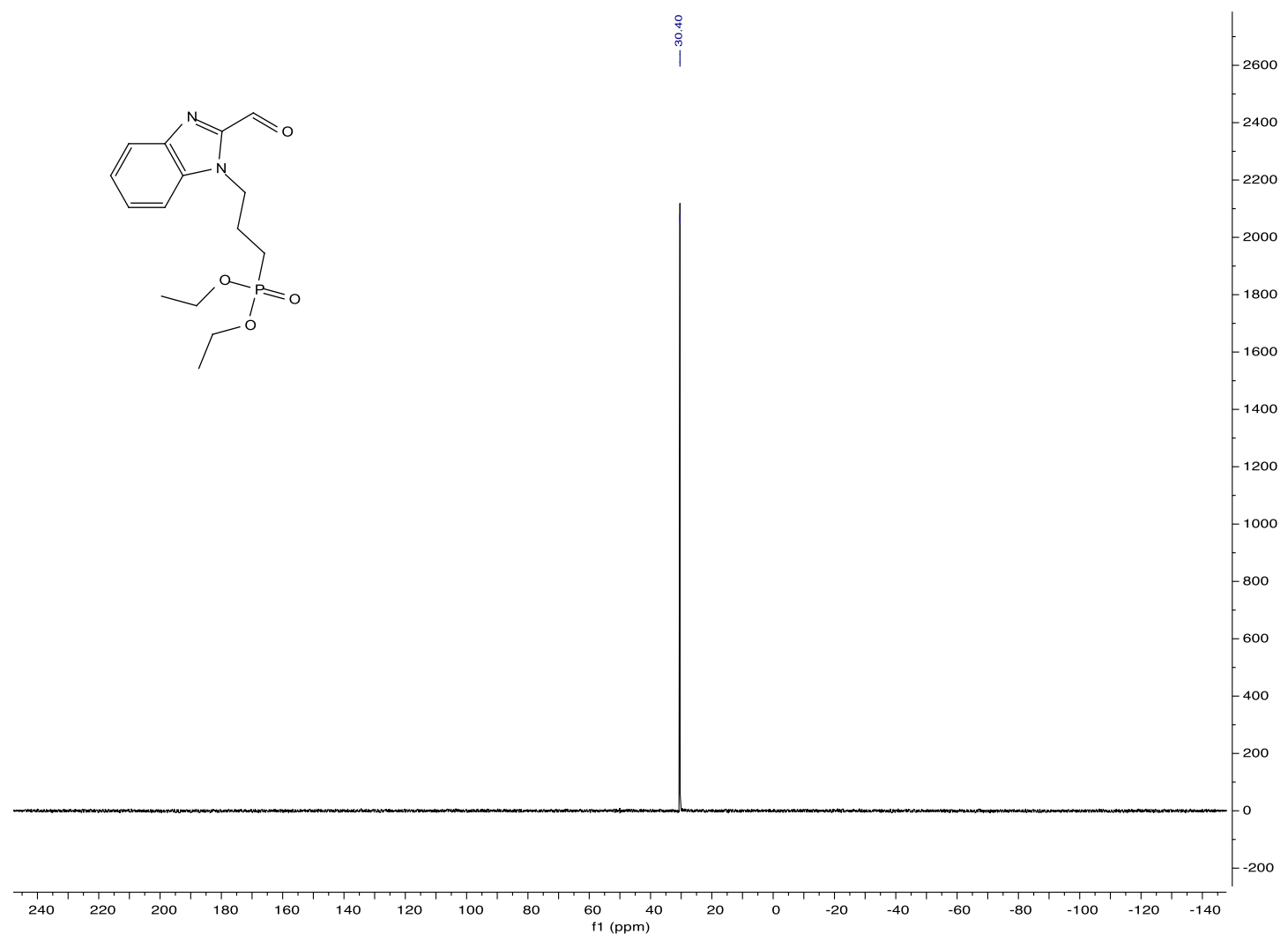

Figure S44. ${ }^{31} \mathrm{P}$ NMR of $\left(\mathbf{B i m C}_{3} \mathbf{P E}\right) \mathbf{C H O}$ in $\mathrm{CDCl}_{3}$.

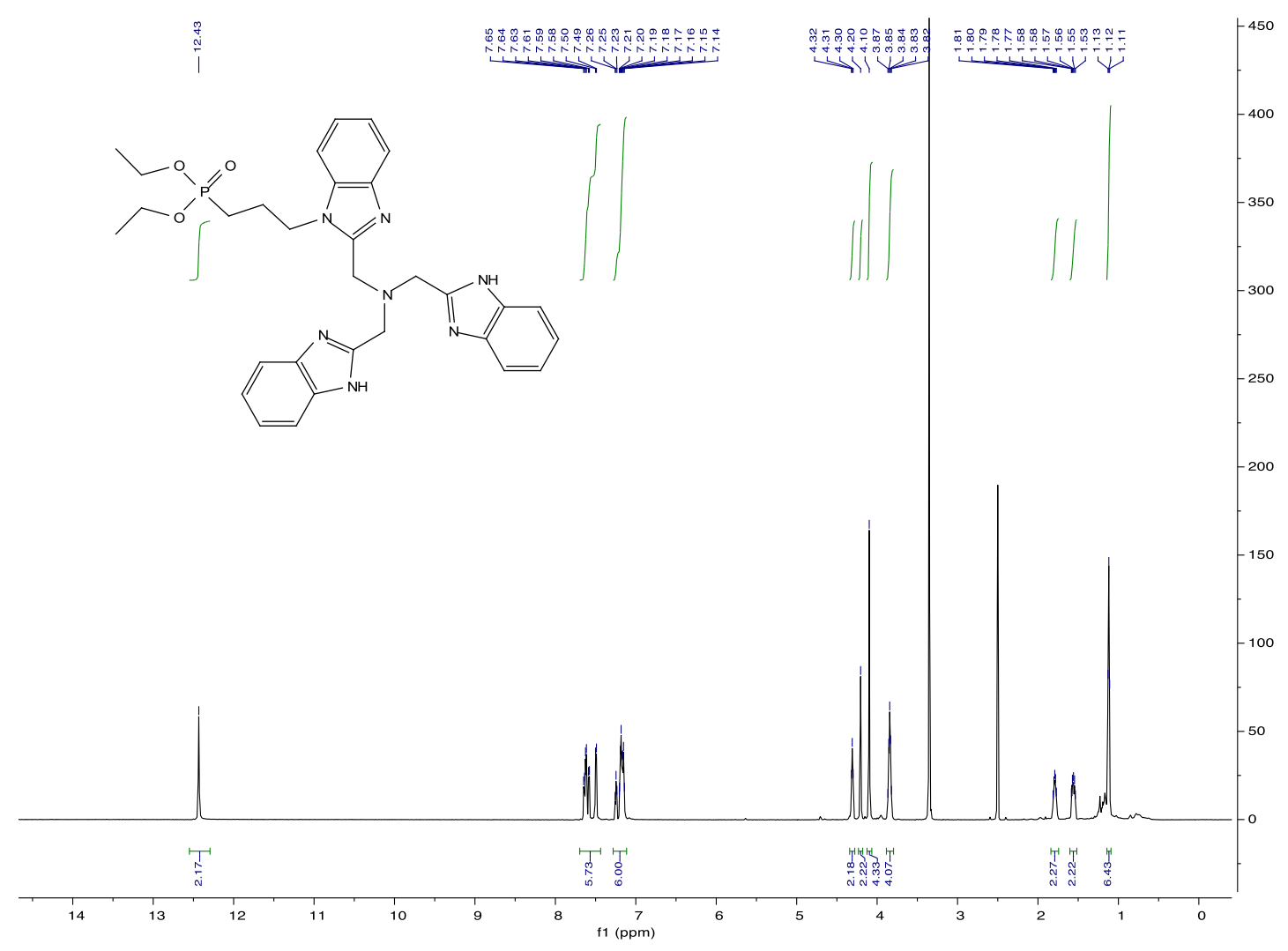

Figure S45. ${ }^{1} \mathrm{H}$ NMR of $(\mathbf{B i m H})_{2}\left(\mathbf{B i m C}_{3} \mathbf{P E}\right)$ in DMSO- $d_{6}$. 


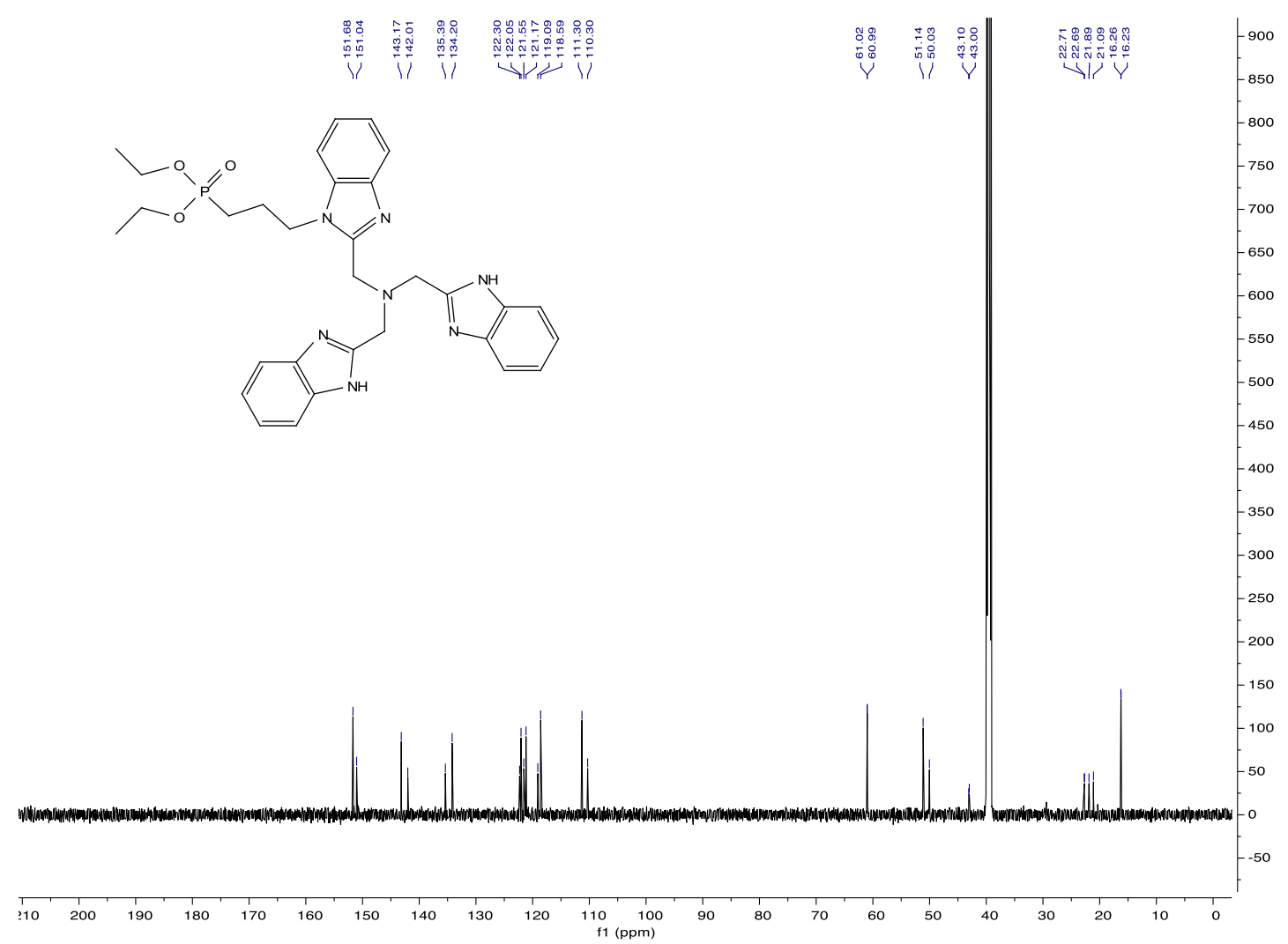

Figure S46. ${ }^{13} \mathrm{C}$ NMR of $(\mathrm{BimH})_{2}\left(\mathrm{BimC}_{3} \mathrm{PE}\right)$ in DMSO- $d_{6}$.

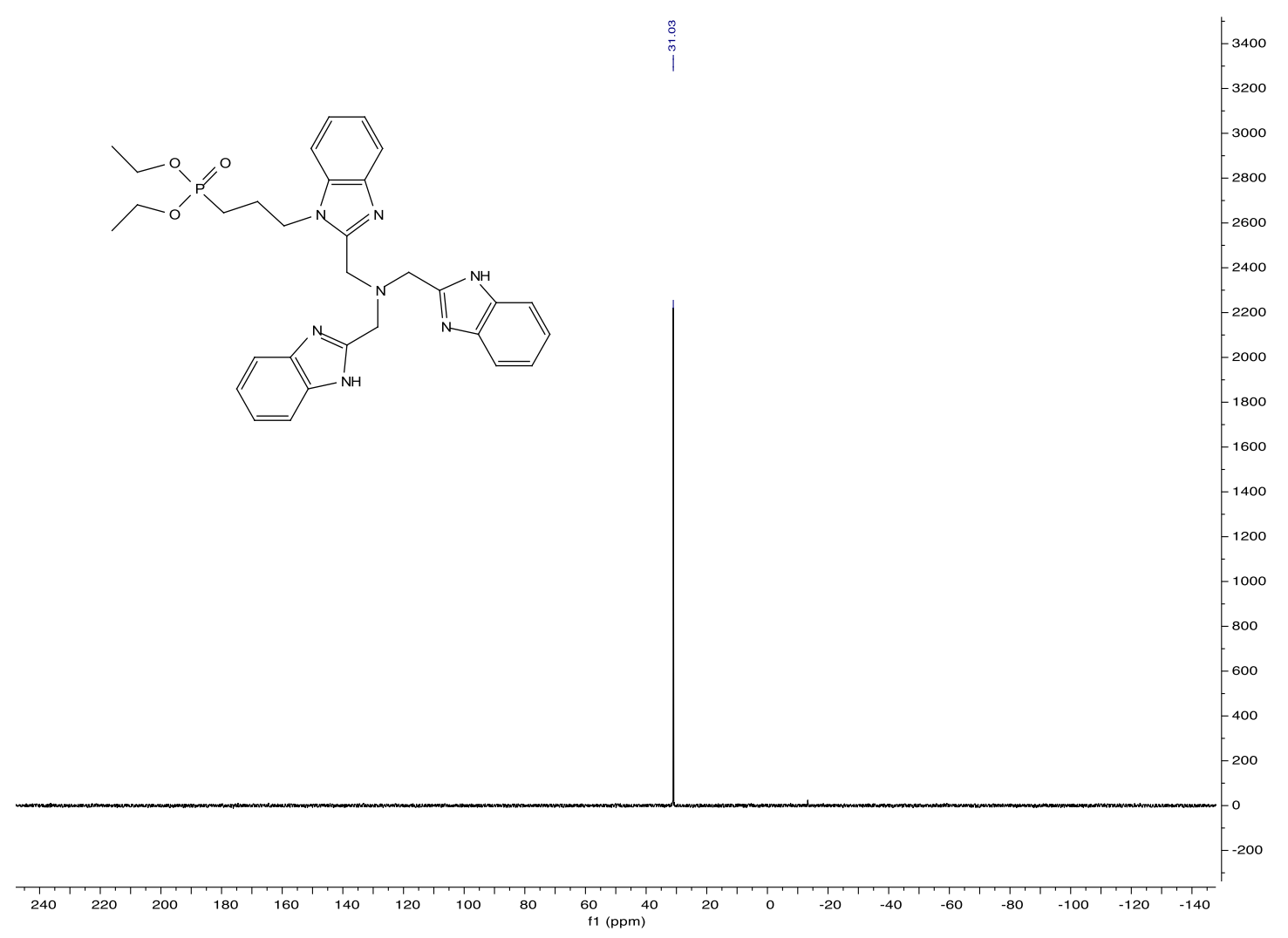

Figure S47. ${ }^{31} \mathrm{P}$ NMR of $(\mathbf{B i m H})_{2}\left(\right.$ BimC $\left._{3} \mathbf{P E}\right)$ in DMSO- $d_{6}$. 


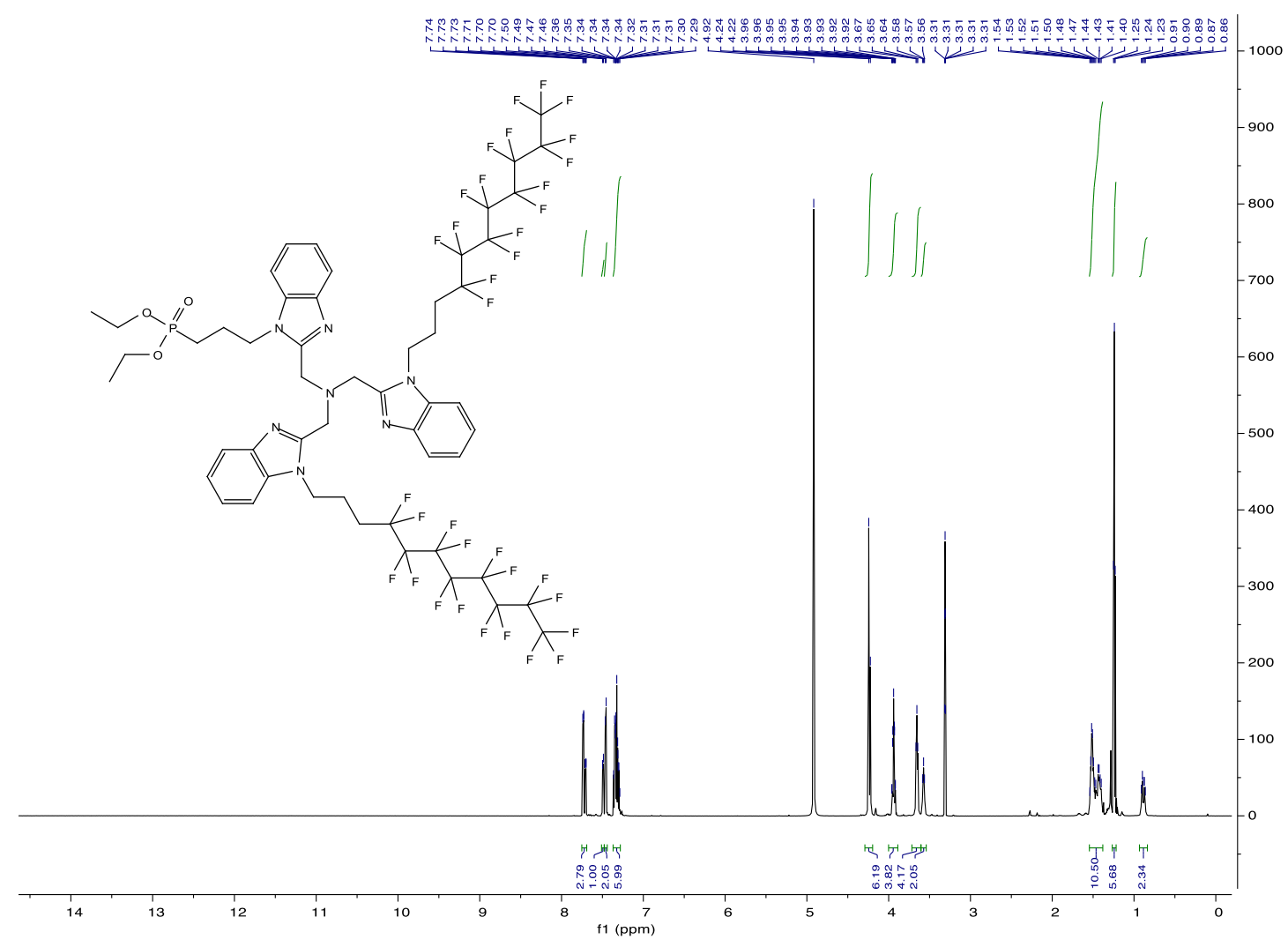

Figure S48. ${ }^{1} \mathrm{H}$ NMR of $\left(\operatorname{BimC}_{3} \mathbf{F}_{8}\right)_{2}\left(\mathbf{B i m C}_{3} \mathbf{P E}\right)$ in methanol- $d_{4}$.

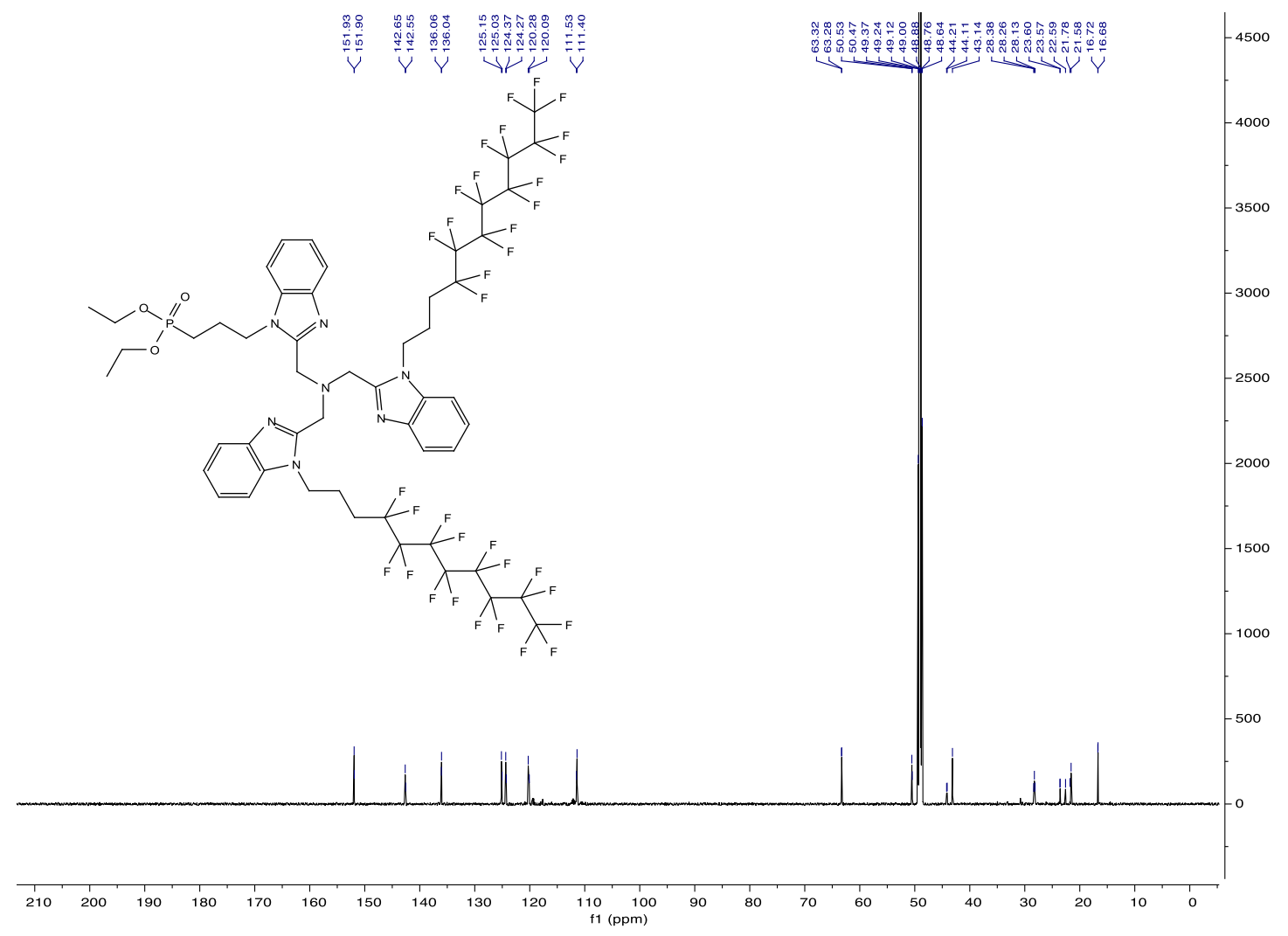

Figure S49. ${ }^{13} \mathrm{C}$ NMR of $\left(\operatorname{BimC}_{3} \mathbf{F}_{8}\right)_{2}\left(\mathbf{B i m C}_{3} \mathbf{P E}\right)$ in methanol- $d_{4}$. 


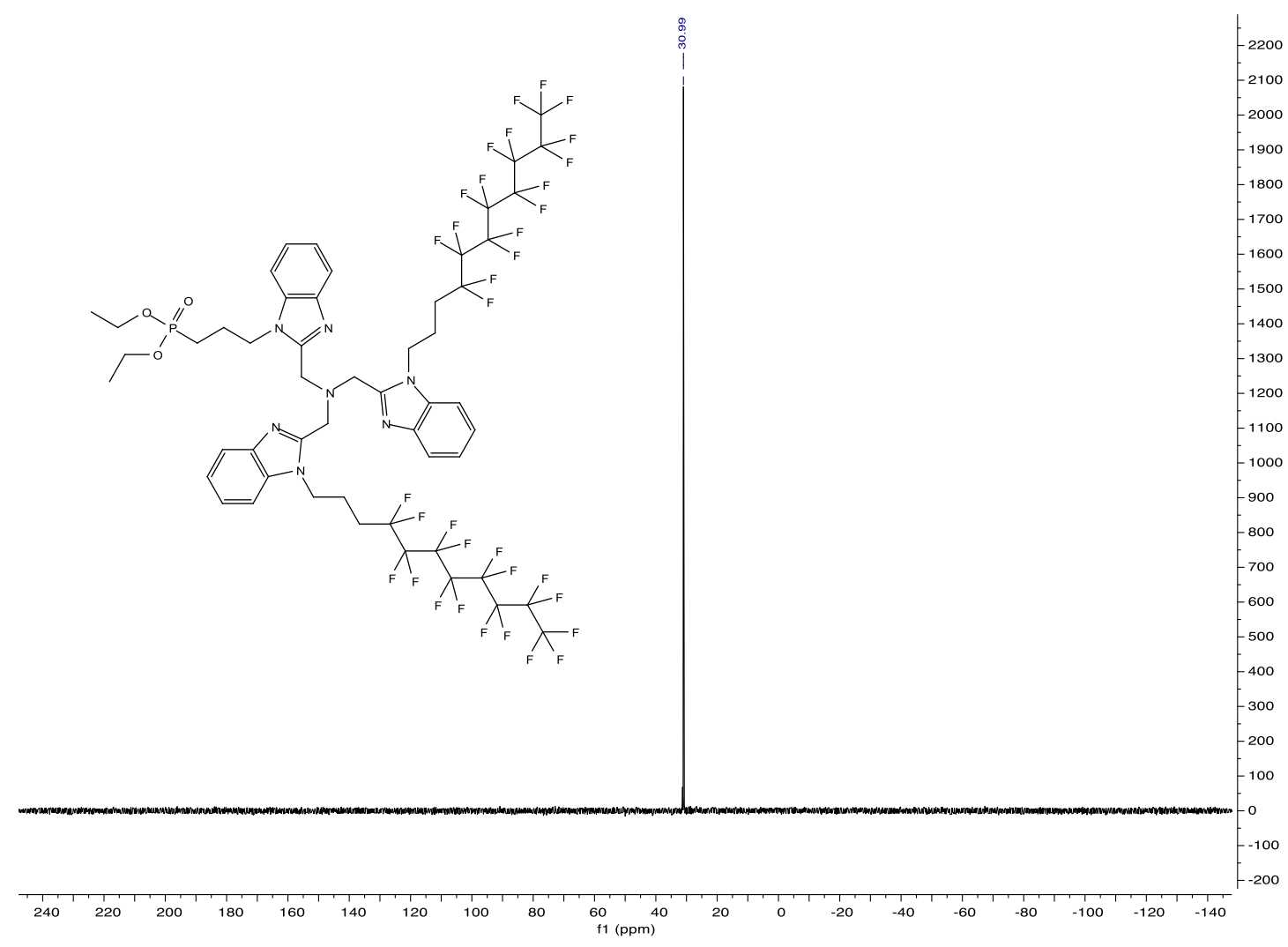

Figure S50. ${ }^{31} \mathrm{P}$ NMR of $\left(\mathrm{BimC}_{3} \mathrm{~F}_{8}\right)_{2}\left(\mathbf{B i m C}_{3} \mathbf{P E}\right)$ in methanol- $d_{4}$.

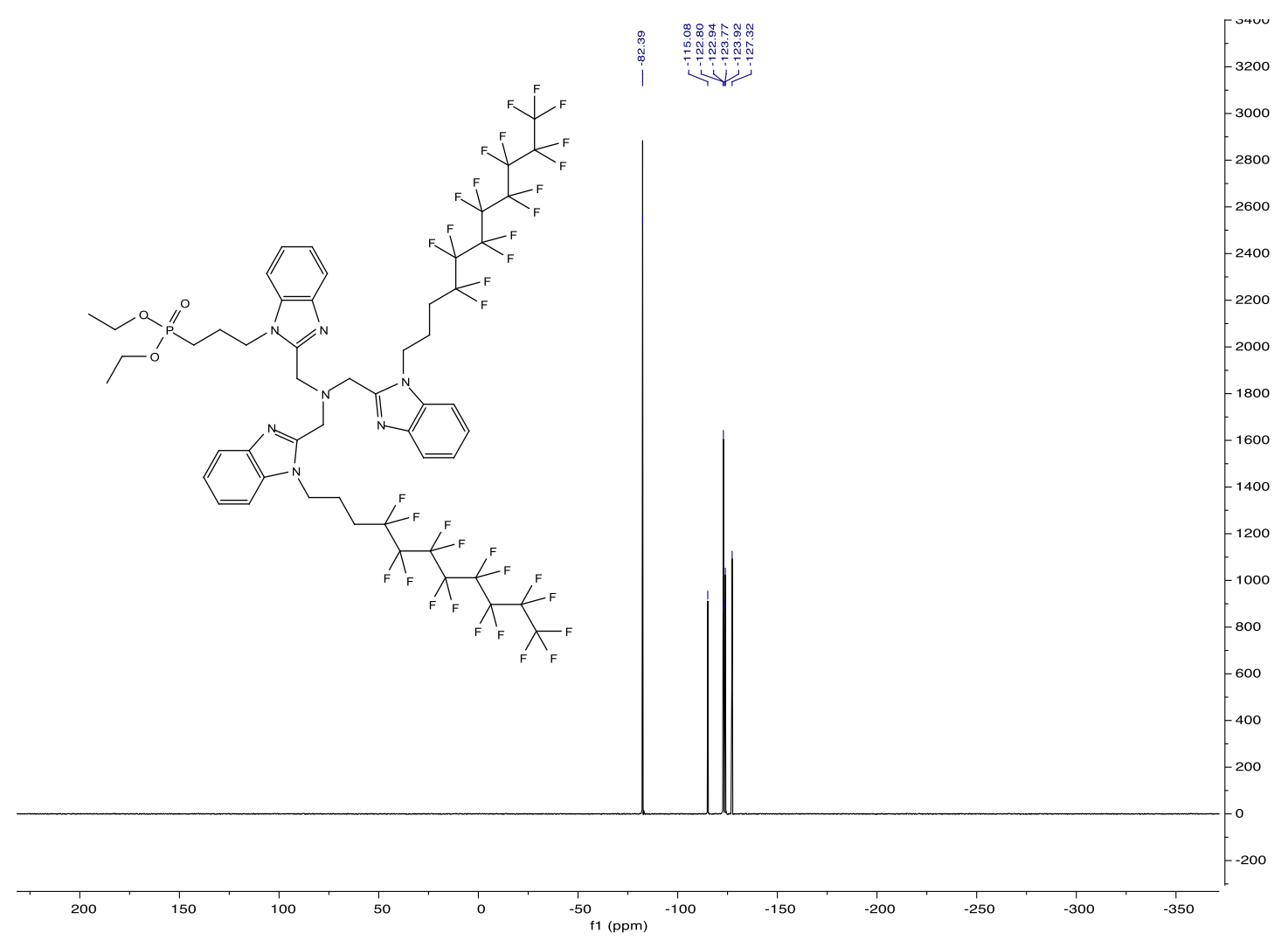

Figure S51. ${ }^{19} \mathrm{~F}$ NMR of $\left(\mathbf{B i m C}_{3} \mathbf{F}_{8}\right)_{2}\left(\mathbf{B i m C}_{3} \mathbf{P E}\right)$ in methanol- $d_{4}$. 


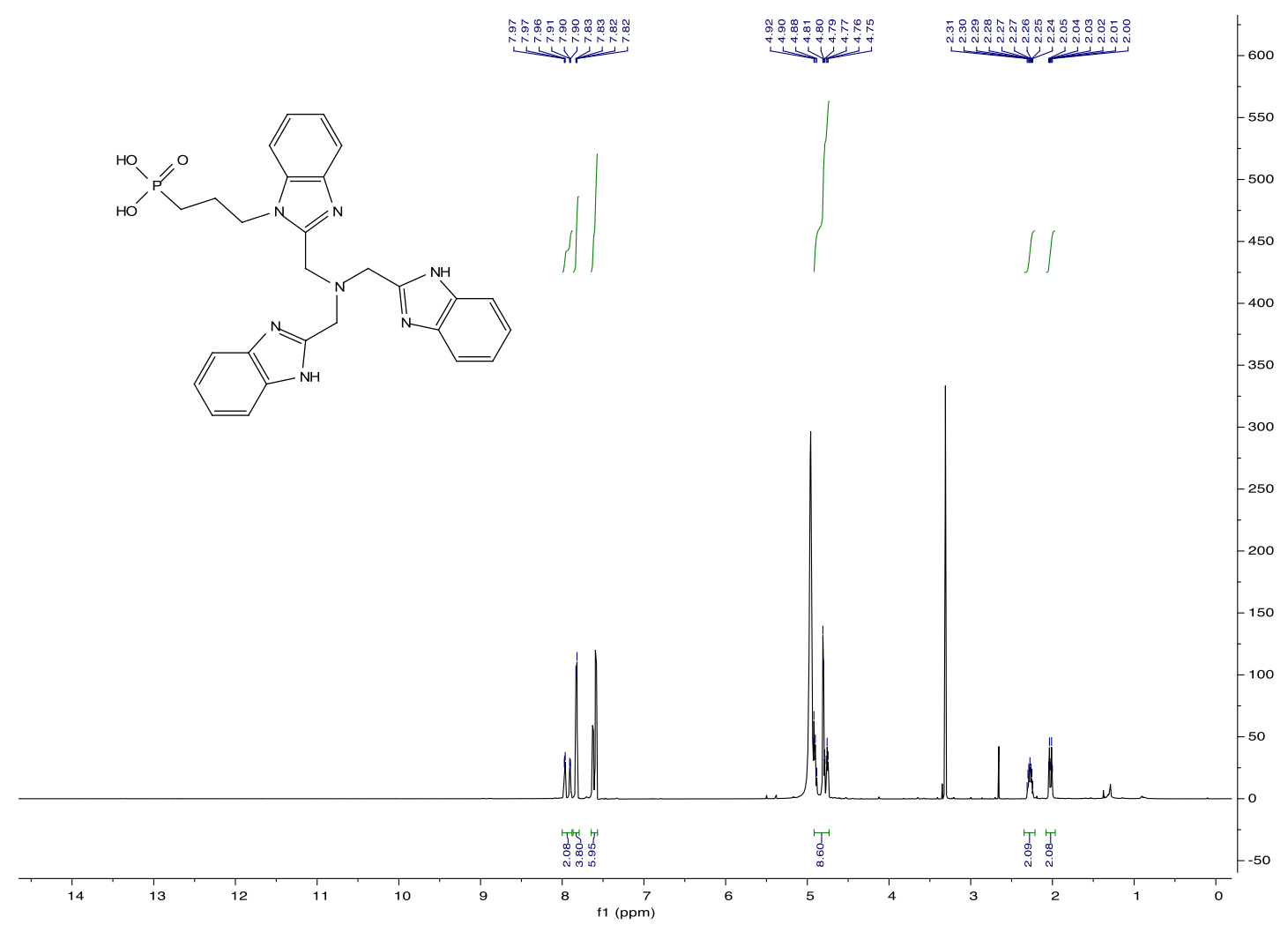

Figure S52. ${ }^{1} \mathrm{H}$ NMR of $(\mathbf{B i m H})_{2}\left(\mathbf{B i m C}_{3} \mathbf{P A}\right)$ in methanol- $d_{4}$.

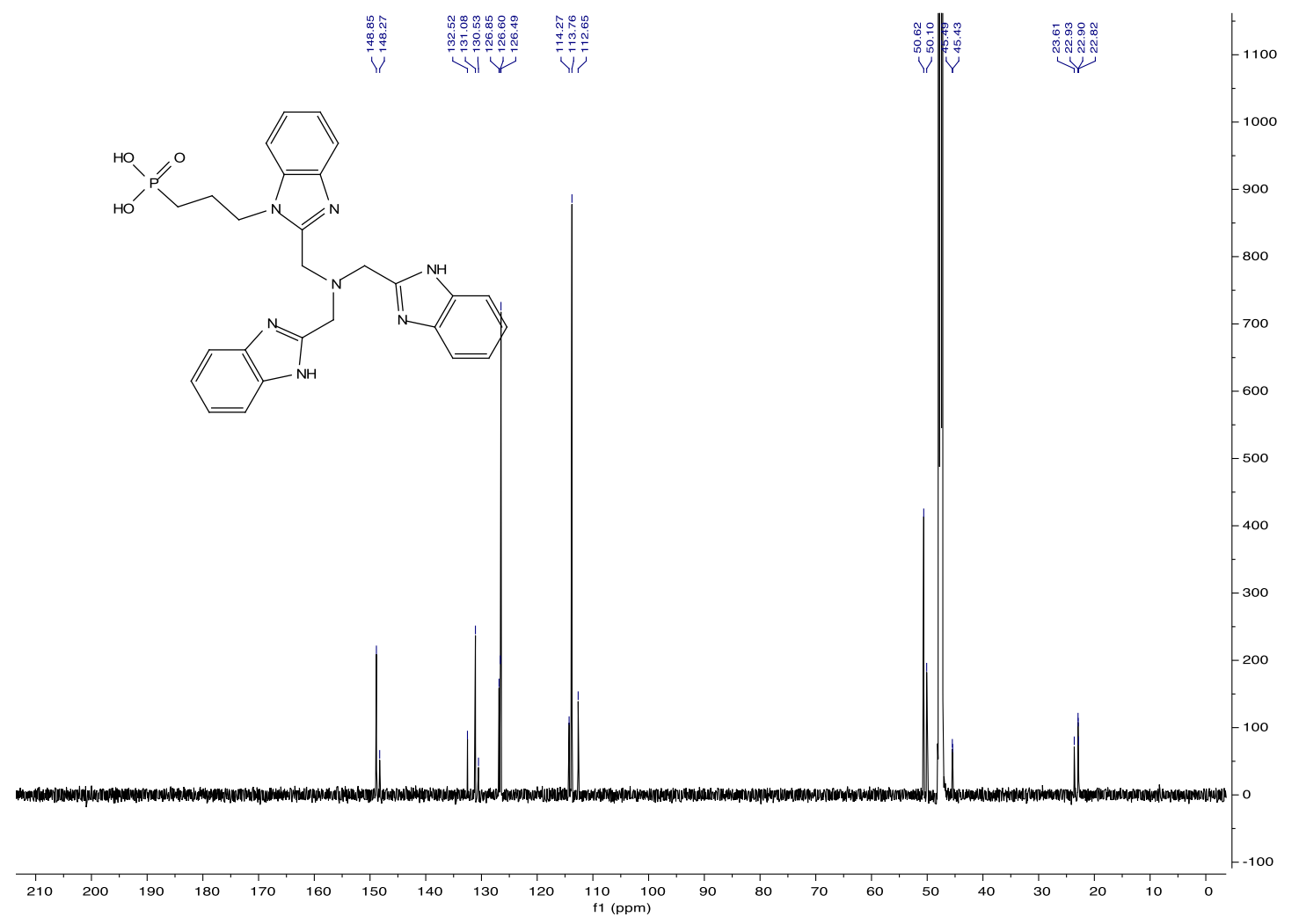

Figure S53. ${ }^{13} \mathrm{C}$ NMR of $(\mathbf{B i m H})_{2}\left(\mathbf{B i m C}_{3} \mathbf{P A}\right)$ in methanol- $d_{4}$. 


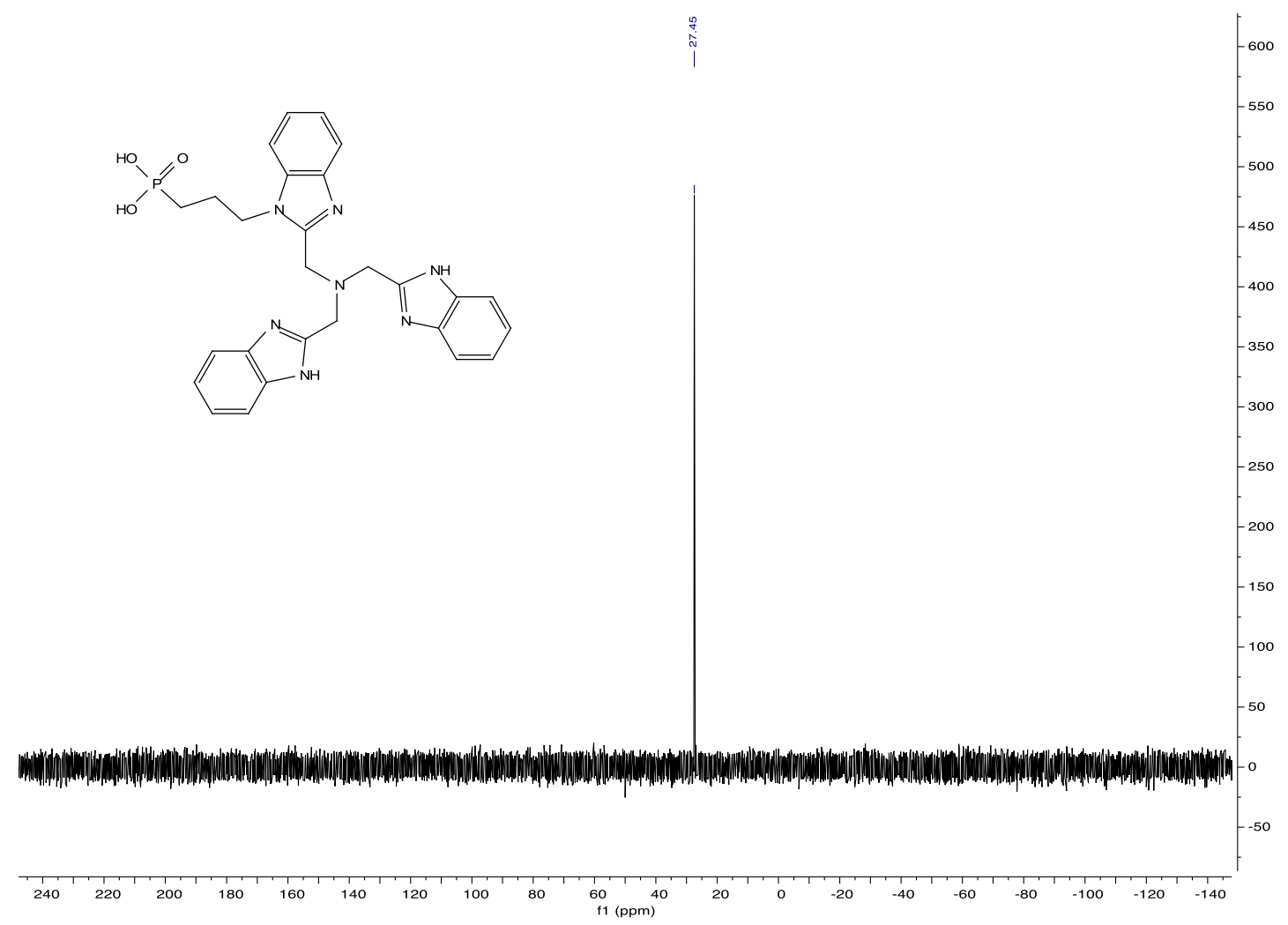

Figure S54. ${ }^{11} \mathrm{P}$ NMR of $(\operatorname{BimH})_{2}\left(\operatorname{BimC}_{3} \mathbf{P A}\right)$ in methanol- $d_{4}$.

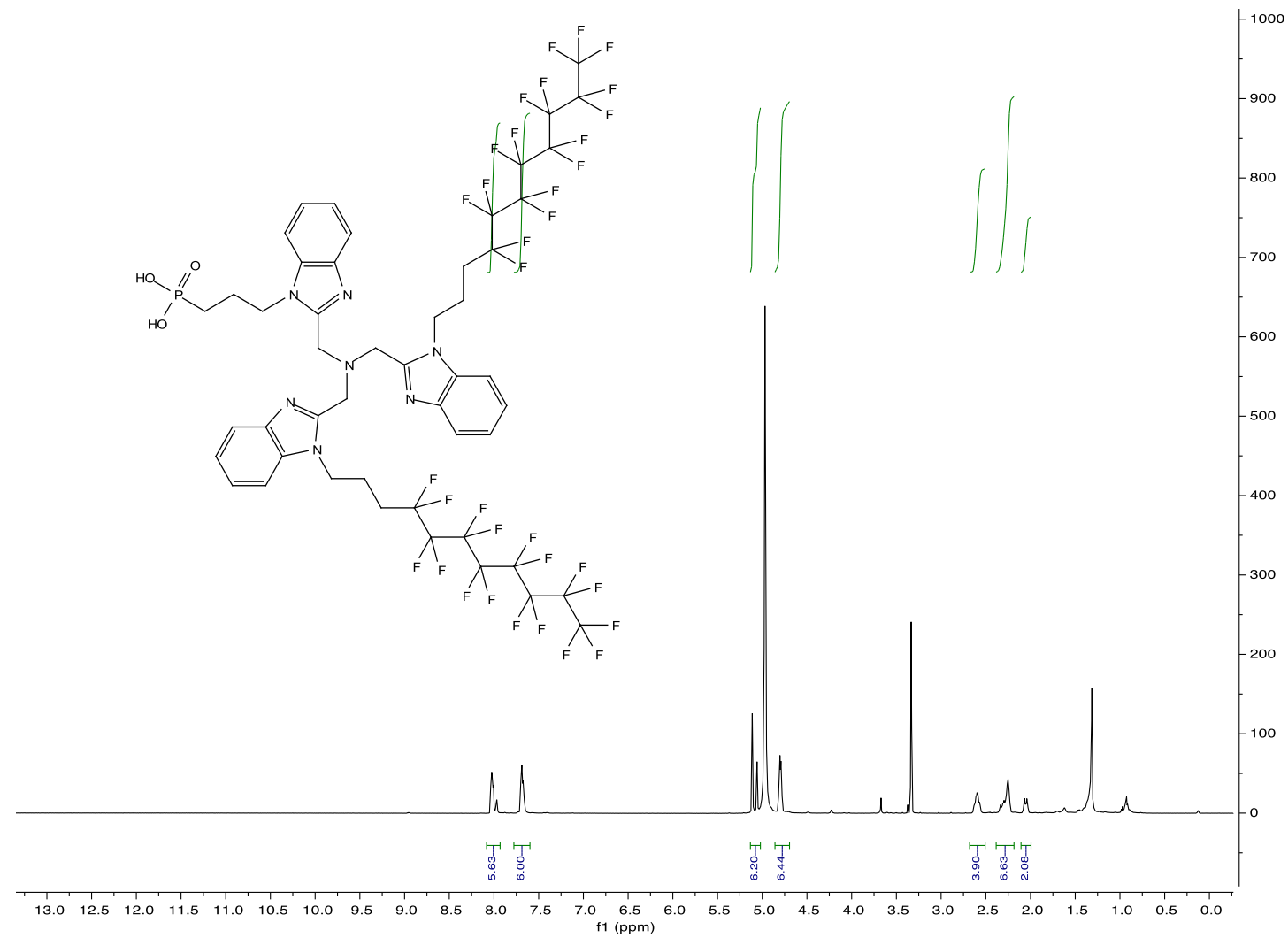

Figure S55. ${ }^{1} \mathrm{H}$ NMR of $\left(\operatorname{BimC}_{3} \mathbf{F}_{8}\right)_{2}\left(\mathbf{B i m C}_{3} \mathbf{P A}\right)$ in methanol- $d_{4}$. 


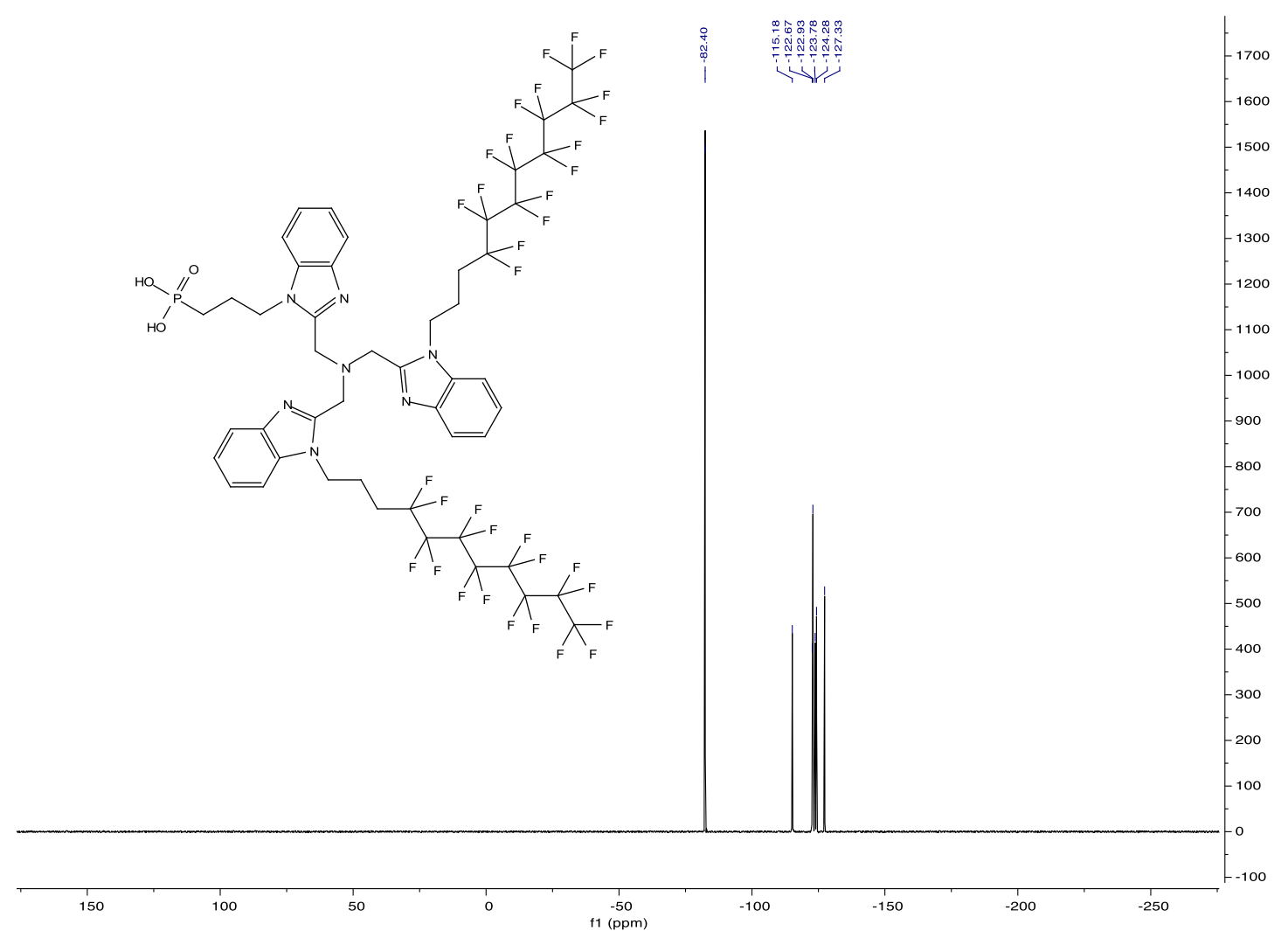

Figure S56. ${ }^{19} \mathrm{~F}$ NMR of $\left(\mathbf{B i m C}_{3} \mathbf{F}_{8}\right)_{2}\left(\mathbf{B i m C}_{3} \mathbf{P A}\right)$ in methanol- $d_{4}$.

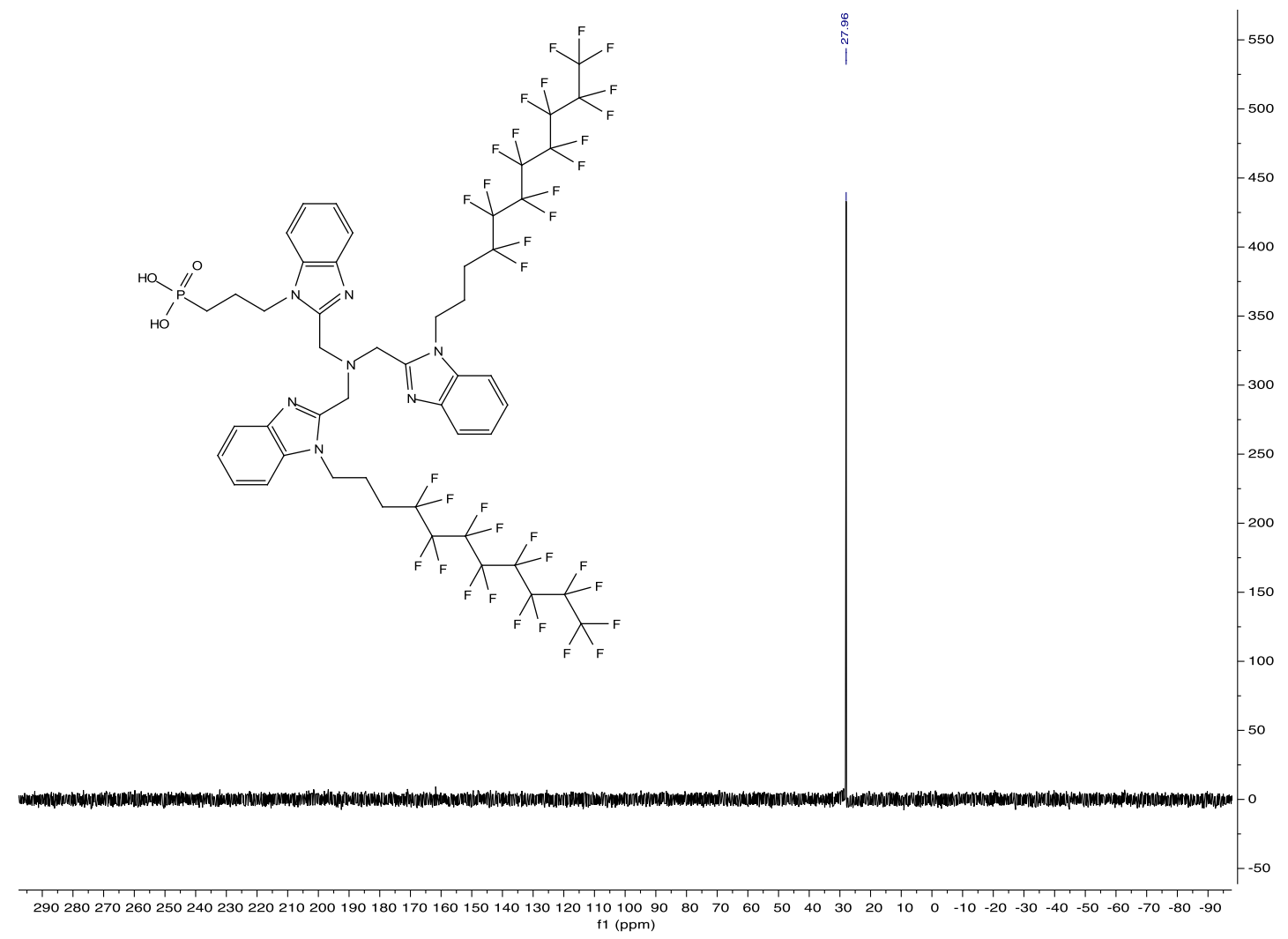

Figure S57. ${ }^{31} \mathrm{P}$ NMR of $\left(\mathbf{B i m C}_{3} \mathbf{F}_{8}\right)_{2}\left(\mathbf{B i m C}_{3} \mathbf{P A}\right)$ in methanol- $d_{4}$. 


\section{References}

(1) Morlanes, N.; Joya, K. S.; Takanabe, K.; Rodionov, V. Eur. J. Inorg. Chem. 2015, 1, 49.

(2) Wang, D.; Groves, J. T. Proc. Natl. Acad. Sci. USA 2013, 110, 15579.

(3) Wasylenko, D. J.; Ganesamoorthy, C.; Borau-Garcia, J.; Berlinguette, C. P. Chem. Commun. 2011 , 47, 4249.

(4) Dogutan, D. K.; McGuire, R., Jr.; Nocera, D. G. J. Am. Chem. Soc. 2011, 133, 9178.

(5) Rigsby, M. L.; Mandal, S.; Nam, W.; Spencer, L. C.; Llobet, A.; Stahl, S. S. Chem. Sci. 2012, 3, 3058.

(6) Stracke, J. J.; Finke, R. G. J. Am. Chem. Soc. 2011, 133, 14872.

(7) Rodionov, V. O.; Presolski, S. I.; Gardinier, S.; Lim, Y. H.; Finn, M. G. J. Am. Chem. Soc. 2007, $129,12696$.

(8) Ichikawa, K.; Nakata, K.; Ibrahim, M. M. Chem. Lett. 2000, 796.

(9) Phillips, M. A. J. Chem. Soc. 1928, 2393.

(10) O'Shaughnessy, J.; Aldabbagh, F. Synthesis 2005, 1069.

(11) Cariou, R.; Chirinos, J. J.; Gibson, V. C.; Jacobsen, G.; Tomov, A. K.; Britovsek, G. J. P.; Whitea, A. J. P. Dalton Trans. 2010, $39,9039$.

(11) APEX2 Version 2014.9-0, SAINT Version 8.34A and SADABS Version 2014/4. Bruker Analytical X-ray Systems, Inc., Madison, Wisconsin, USA, 2014.

(12) (a) SHELXT: Sheldrick, G.M., Acta Cryst. 2015, A71, 3-8. (b) SHELXL: Sheldrick, G.M., Acta Cryst. 2008, A64, $112-122$.

(13) Dolomanov, O. V.; Bourhis, L. J.; Gildea, R. J.; Howard J. A. K.; Puschmann, H. J., Appl. Cryst. 2009, 42, $339-341$. 